THE DENSITY FIELD OF THE 10k zCOSMOS GALAXIES

This article has been downloaded from IOPscience. Please scroll down to see the full text article. 2010 ApJ 708505

(http://iopscience.iop.org/0004-637X/708/1/505)

The Table of Contents and more related content is available

Download details:

IP Address: 193.206.157.4

The article was downloaded on 24/03/2010 at 09:19

Please note that terms and conditions apply. 


\title{
THE DENSITY FIELD OF THE 10k zCOSMOS GALAXIES*
}

\author{
K. Kovač ${ }^{1}$, S. J. Lilly ${ }^{1}$, O. Cucciati ${ }^{2,3}$, C. Porciani ${ }^{1,4}$, A. Iovino ${ }^{2}$, G. Zamorani ${ }^{5}$, P. Oesch ${ }^{1}$, M. Bolzonella ${ }^{5}$, C. Knobel ${ }^{1}$, \\ A. Finoguenov ${ }^{6}$, Y. Peng ${ }^{1}$, C. M. Carollo ${ }^{1}$, L. Pozzetti ${ }^{5}$, K. Caputi ${ }^{1}$, J. D. Silverman ${ }^{1}$, L. A. M. Tasca ${ }^{3,7}$, M. Scodeggio ${ }^{7}$, \\ D. Vergani ${ }^{5}$, N. Z. Scoville ${ }^{8}$, P. CAPAK ${ }^{8,9}$, T. Contini ${ }^{10}$, J.-P. KNeib ${ }^{3}$, O. Le Fèvre ${ }^{3}$, V. MAinieri ${ }^{11}$, A. RenZini ${ }^{12}$, \\ S. Bardelli ${ }^{5}$, A. Bongiorno ${ }^{6}$, G. Coppa ${ }^{5}$, S. DE la Torre ${ }^{2,3,7}$, L. De Ravel ${ }^{3}$, P. Franzetti ${ }^{7}$, B. Garilli ${ }^{7}$, L. Guzzo ${ }^{2}$, \\ P. KampczyK ${ }^{1}$, F. Lamareille ${ }^{10}$, J.-F. Le Borgne ${ }^{10}$, V. Le Brun ${ }^{3}$, C. Maier ${ }^{1}$, M. Mignoli ${ }^{5}$, R. Pello ${ }^{10}$, E. Perez Montero ${ }^{10}$, \\ E. Ricciardelli ${ }^{12}$, M. Tanaka ${ }^{11}$, L. Tresse ${ }^{3}$, E. Zucca $^{5}$, U. Abbas $^{3,13}$, D. Bottini $^{7}$, A. Cappi ${ }^{5}$, P. Cassata $^{3}$, A. Cimatti ${ }^{14}$, \\ M. Fumana ${ }^{7}$, A. M. Koekemoer ${ }^{15}$, D. Maccagni ${ }^{7}$, C. Marinoni ${ }^{16}$, H. J. McCracken ${ }^{17}$, P. MemeO ${ }^{7}$, B. MeneuX ${ }^{6,18}$, \\ AND R. SCARAMELLA 19 \\ ${ }^{1}$ Institute of Astronomy, ETH Zürich, CH-8093, Zürich, Switzerland \\ ${ }^{2}$ INAF Osservatorio Astronomico di Brera, Milan, Italy \\ ${ }^{3}$ Laboratoire d'Astrophysique de Marseille, Marseille, France \\ ${ }^{4}$ Argelander Institut für Astronomie, Auf dem Hügel 71, D-53121 Bonn, Germany \\ ${ }^{5}$ INAF Osservatorio Astronomico di Bologna, via Ranzani 1, I-40127, Bologna, Italy \\ ${ }^{6}$ Max-Planck-Institut für extraterrestrische Physik, D-84571 Garching, Germany \\ ${ }^{7}$ INAF-IASF Milano, Milan, Italy \\ ${ }^{8}$ California Institute of Technology, MS 105-24, Pasadena, CA 91125, USA \\ ${ }^{9}$ Spitzer Science Center, 314-6 Caltech, Pasadena, CA 91125, USA \\ ${ }^{10}$ Laboratoire d'Astrophysique de Toulouse-Tarbes, Universite de Toulouse, CNRS, 14 avenue Edouard Belin, F-31400 Toulouse, France \\ ${ }^{11}$ European Southern Observatory, Karl-Schwarzschild- Strasse 2, Garching, D-85748, Germany \\ ${ }^{12}$ Dipartimento di Astronomia, Universita di Padova, Padova, Italy \\ ${ }^{13}$ INAF Osservatorio Astronomico di Torino, Strada Osservatorio 20, I-10025 Pino Torinese, Turin, Italy \\ ${ }^{14}$ Dipartimento di Astronomia, Universitá di Bologna, via Ranzani 1, I-40127, Bologna, Italy \\ ${ }^{15}$ Space Telescope Science Institute, 3700 San Martin Drive, Baltimore, MD 21218, USA \\ ${ }^{16}$ Centre de Physique Theorique, Marseille, Marseille, France \\ ${ }^{17}$ Institut d'Astrophysique de Paris, UMR 7095 CNRS, Université Pierre et Marie Curie, 98 bis Boulevard Arago, F-75014 Paris, France \\ ${ }^{18}$ Universitats-Sternwarte, Scheinerstrasse 1, D-81679 Muenchen, Germany \\ ${ }^{19}$ INAF, Osservatorio di Roma, Monteporzio Catone (RM), Italy \\ Received 2008 November 28; accepted 2009 November 10; published 2009 December 14
}

\begin{abstract}
We use the current sample of $\sim 10,000$ zCOSMOS spectra of sources selected with $I_{\mathrm{AB}}<22.5$ to define the density field out to $z \sim 1$, with much greater resolution in the radial dimension than has been possible with either photometric redshifts or weak lensing. We present the new algorithm that we have developed (ZADE) to incorporate objects not yet observed spectroscopically by modifying their photometric redshift probability distributions using the spectroscopic redshifts of nearby galaxies. We present a number of tests on mock catalogs used to justify this approach. The ZADE algorithm allows us to probe a broader range of galaxy environments and reduce the Poisson noise in the density field. The reconstructed overdensity field of the 10k zCOSMOS galaxies consists of cluster-like patterns surrounded by void-like regions, extending up to $z \sim 1$. Some of these structures are very large, spanning the $\sim 50 \mathrm{~h}^{-1} \mathrm{Mpc}$ transverse direction of the COSMOS field and extending up to $\Delta z \sim 0.05$ in redshift. We present the three-dimensional overdensity maps and compare the reconstructed overdensity field to the independently identified virialized groups of galaxies and clusters detected in the visible and in X-rays. The distribution of the overdense structures is in general well traced by these virialized structures. A comparison of the large-scale structures in the zCOSMOS data and in the mock catalogs reveals an excellent agreement between the fractions of the volume enclosed in structures of all sizes above a given overdensity between the data and the mocks in $0.2<z<1$, although in the data these overdense regions are in generally larger contiguous structures.
\end{abstract}

Key words: galaxies: high-redshift - large-scale structure of universe - surveys

Online-only material: color figures

\section{INTRODUCTION}

Although on scales larger than $\sim 100 h^{-1}$ Mpc the universe is homogeneous and isotropic, on smaller scales structures in the universe are organized hierarchically. The full view of the cosmic structure was revealed for the first time in the first CfAII slice (de Lapparent et al. 1986), with galaxies distributed in bubble-like structures surrounding empty regions. Later surveys, particularly the Sloan Digital Sky Survey (SDSS; York

\footnotetext{
* Based on observations obtained at the European Southern Observatory (ESO) Very Large Telescope (VLT), Paranal, Chile, as part of the Large Program 175.A-0839 (the zCOSMOS Spectroscopic Redshift Survey).
}

et al. 2000) and the two-degree Field Galaxy Redshift Survey (2dFGRS; Colless et al. 2001), have confirmed the highly complex hierarchical picture of the universe at $z \sim 0$. Our universe exhibits a range of cellular structures, the cosmic web (Bond et al. 1996), where galaxies define the structures which form patterns of dense compact clusters, elongated filaments, and sheet-like walls outlining large and almost empty voids. While the network of filaments and clumps sitting in the intersection of filaments dominate the visual impression of the cosmic web, most of the volume is in the underdense regions (voids).

The picture emerging from the surveys of the higher redshift universe is limited by small fields and cosmic variance, but the richness and complexity of the cosmic web appears (at 
least visually) to match that of the local universe out to $z \sim 1.5$ (e.g., Scoville et al. 2007b; Marinoni et al. 2008). As redshift increases, the peak in the probability distribution function (PDF) of the galaxy overdensity field shifts toward lower overdensity values, and the fraction of the volume in the underdense regions decreases (Marinoni et al. 2008).

The galaxy distribution is believed to be a (biased) tracer of the underlying smooth and continuous matter density field. It has been shown by numerical simulations and by analytical work that the observed large-scale structure (LSS) pattern is a natural manifestation of the gravitational structure formation process through the amplification of density fluctuations and their subsequent collapse. The presence of the cosmic web can be explained with the tendency of the matter concentrations to contract and collapse gravitationally in an anisotropic manner (Bond et al. 1996; van de Weygaert \& Bertschinger 1996).

One of the main characteristics of the cosmic web is its hierarchical nature. The LSSs cover a broad range in (over) density values, physical scales, and geometrical shapes. The reconstruction of the density field from an observed galaxy sample should ideally preserve all these features. However, the samples of the galaxies are incomplete, particularly at high redshifts, and the sampling is often irregular. Different galaxy populations evolve with redshift in different ways, and they may trace the underlying mass distribution in different ways at different redshifts. On top of this, one has to add an uncertainty in the measured redshifts, magnitudes, and other galaxy properties. All of these points make the reconstruction of the density field in the universe non-trivial.

The reconstruction of a continuous, presumably smooth field from a set of measured discrete data points usually involves the interpolation and smoothing of the data with some filtering function into a continuous map. Unlike the velocity or temperature fields for which we sample the field values in the observed points, for the density field reconstruction there are no field samples available. The measured data points (galaxies) are used to define the density field itself. A common feature of all the available approaches to reconstruct the density field is that the results of the reconstructed density field depend on the method used to carry out the reconstruction. Usually, a particular scientific application guides the choice of the method to reconstruct the density field, and we briefly review this here.

\subsection{Applications of the Density Field}

\subsubsection{Extraction of the Components of the Cosmic Web}

An immediate application of the reconstructed density field is the extraction of the four components of the cosmic web (clumps, filaments, sheets, and voids). While the human eye can easily distinguish between these shapes, there does not exist an ideal algorithm yet to extract these features in an algorithmically well-defined way. A lot of progress has been made recently, by applying a geometrical classification directly to the density field, such as the multi-scale analysis of the Hessian matrix of the density field (Aragón-Calvo et al. 2007) or the skeleton analysis of the density field (Novikov et al. 2006; Sousbie et al. 2008). Alternatively, a classification of the cosmic web can be done using either a linear (Lee \& Lee 2008) or nonlinear (Hahn et al. 2007a, 2007b; Forero-Romero et al. 2009) gravitational potential. Some of these methods have been applied so far only on numerical simulations and result in the classification of the dark matter structures. The reconstruction of the density field from observed galaxy data is based on a very much smaller number of objects, and therefore it lacks the spatial resolution of the simulations. However, probably the most difficult task to tackle is that the observations provide information on the position of galaxies in redshift space, while for the proper reconstruction of the cosmic web, galaxy positions need to be measured in real space (Lee \& Lee 2008; Lee \& Li 2008). This is not of course a problem in simulations.

\subsubsection{Correlation between Galaxies and Environment in which They Reside}

Several correlations between galaxy properties and the environments in which they reside are observed in the local universe. In this context, the environment of a galaxy is given by the number density (or overdensity) of the neighboring galaxies. It has been known for many years that elliptical and lenticular galaxies reside in more dense environments than spiral galaxies (e.g., Dressler 1980). Red, older, less star-forming galaxies live in more dense regions than blue, younger galaxies with high star formation rates (e.g., Kauffmann et al. 2004; Blanton et al. 2006). These trends are observed in environments ranging from clusters to voids.

Are the early conditions of galaxy formation (the so-called nature scenario) or the environment in which they subsequently reside (the so-called nurture scenario), or both of these, responsible for the observed dependences of galaxy properties on environment? An obvious way to tackle this question is to go to higher redshifts and to establish at which redshift the relations in question are in place. Not long ago, high redshift observations were limited to the most dense structures (i.e., clusters) or targeted to detect a specific galaxy population (e.g., Lyman break galaxies or Ly $\alpha$ emitters). Large and deep redshift surveys are the best probes of the (over-)density field delineated by galaxies at all redshifts. The recent spectroscopic high redshift surveys, the DEEP2 Galaxy Redshift Survey (Davis et al. 2003) and the VIMOS VLT Deep Survey (VVDS) (Le Fèvre et al. 2005) and now zCOSMOS (Lilly et al. 2007, 2009), sample the broad range of galaxy population and allow the continuous reconstruction of galaxy environments. Most of the galaxy property-environment relations observed at $z \sim 0$ seems to be at place already at $z \sim 1$ (e.g., Cucciati et al. 2006; Cooper et al. 2007).

Even in the local universe, there is an ongoing debate on whether the observed galaxy-environment relations depend on the particular scale at which the environment has been measured. This would potentially give a clue to their origin. Kauffmann et al. (2004) and Blanton et al. (2006) both find that only the environment measured on the small scales (of $1 h^{-1} \mathrm{Mpc}$ ) appears to affect the star formation histories of galaxies. In the context of the cold dark matter (CDM) scenario, this scale corresponds to the scale of the individual dark matter halo in which galaxy resides, whereas the larger scale would probe more the location in the cosmic web.

In the current state-of-the-art of the high redshift surveys, it is very challenging to reconstruct environments on such a small scale. Galaxies targeted for the observations at higher redshifts are generally more luminous, and their mean intergalaxy separations are thus larger. Furthermore, only a fraction of galaxies is targeted for the observations, and this fraction may be substantially smaller than locally.

\subsubsection{Galaxy Density Field as Biased Tracer of the Matter Density Field}

In the current cosmological picture, it is almost a paradigm that galaxies are a biased tracer of the underlying matter 
distribution. The biasing factor can be a non-trivial function of the scale, of the redshift, and of the type of galaxies used to reconstruct the density field. Using a statistical approach, one of the ways to infer the biasing function is from a comparison of the PDFs of the galaxy and matter overdensities (Sigad et al. 2000; Marinoni et al. 2005). While the first is based on observations, the PDF of the matter comes from theory and is dependent on the assumed cosmological parameters. In the case of the three-dimensional overdensities reconstructed with a top-hat filter, the PDF of the matter is well described by the log-normal distribution (Coles \& Jones 1991).

\subsection{Goals of this Study}

The main goal of this paper is to reconstruct the galaxy overdensity field in the zCOSMOS region. We reconstruct the overdensity field using the first $\sim 10,000$ spectra of galaxies from the zCOSMOS survey, up to redshift $z \sim 1$ (Lilly et al. 2009), the so-called $10 \mathrm{k}$ sample. High sampling rate and measurements of redshifts with a precision of about $100 \mathrm{~km} \mathrm{~s}^{-1}$ enable us to delineate the environments of galaxies from the $100 \mathrm{kpc}$ scale of galaxy groups - the scale of environment expected to dominate the various galaxy evolutionary processes-up to the $100 \mathrm{Mpc}$ scales of the cosmic web. It is one of the major scientific drivers of the zCOSMOS survey to study the role of environment on galaxy evolution up to high redshifts. We therefore pay particular attention to reconstruct the density field on scales that are as small as possible. With this aim, we present a new method (ZADE), which has been developed to reconstruct the density field using both galaxies with spectroscopic redshifts, and those with only photometric redshifts. The photometric PDFs are modified depending on the proximity of galaxies with high-quality spectroscopic redshifts. We utilize this method to reconstruct the overdensity field both at the positions of the 10k zCOSMOS galaxies and also at any random point in the zCOSMOS volume. The reconstruction has been carried out in a number of ways so as to facilitate a wide range of scientific explorations as discussed above. We present the three-dimensional overdensity maps and compare the LSSs in the overdensity field to the independently estimated virialized structures in the volume of the zCOSMOS survey. Extensive use is made of COSMOS mock catalogs (Kitzbichler \& White 2007) to justify and tune the ZADE method and to estimate the errors on the reconstructed overdensity field. We also use the mock catalogs to compare the zCOSMOS overdensity field and the detected LSSs to those obtained from the mock catalogs. Throughout this paper, we assume a flat cosmology described with $\Omega_{m, 0}=0.25$ and $H_{0}=70 \mathrm{Mpc}^{-1}$. However, we express the results related to the overdensity field using $h, H_{0}=100 \mathrm{~h}$, while stellar masses and absolute magnitudes of galaxies are quoted with an explicitly incorporated $H_{0}=70 \mathrm{Mpc}^{-1}$.

This paper extends the previous studies of the LSS in the COSMOS field based on the photometric redshifts (Scoville et al. 2007b; Guzzo et al. 2007) and weak lensing (Massey et al. 2007). The density field reconstructed in this paper is the basis for rather a large number of studies undertaken by the zCOSMOS collaboration. In follow-up papers, we study the dependence of star formation properties (Cucciati et al. 2009) and morphology (Tasca et al. 2009) on the environments of the $10 \mathrm{k}$ zCOSMOS galaxies presented in this paper. The environmental dependence of the luminosity and mass functions is discussed in Zucca et al. (2009) and Bolzonella et al. (2009), respectively. The biasing function between the
zCOSMOS and matter overdensity fields is presented in Kovač et al. (2009). Other studies cover environmental dependence of specific galaxy populations, such as infrared (IR) galaxies (Caputi et al. 2009), active galactic nuclei (AGN; Silverman et al. 2009) or poststarburst galaxies (Vergani et al. 2009).

\section{THE (GALAXY) DENSITY FIELD RECONSTRUCTION}

A number of methods exist to evaluate the density field, often developed for numerical simulations, e.g., grid based methods, smooth particle hydrodynamics (SPH) like methods, Voronoi and Delaunay tessellation field estimators (VTFE and DTFE). Here, we concentrate on the problem of reconstruction of the density field from the observational data sample of discrete galaxies.

In practice, reconstruction of the galaxy density field (or galaxy environments) reduces to the (weighted) count of objects within some aperture around a set of positions where the density field is to be evaluated. In the general case, the density at an observationally defined position $\mathbf{r}=\mathbf{r}(\alpha, \delta, z)$ can be estimated as

$$
\rho(\mathbf{r})=\Sigma_{i} \frac{m_{i} W\left(\left|\mathbf{r}-\mathbf{r}_{\mathbf{i}}\right| ; R\right)}{\phi\left(\mathbf{r}_{\mathbf{i}}\right)} .
$$

The above equation gives the value of a mean density at a position $\mathbf{r}$ in the redshift space (averaged over the aperture in which the density field is measured), with $W$ being a spatial window function, $m_{i}$ a weight based on the astrophysical properties for each galaxy, and $\phi$ a function correcting for various observational issues. The difference $\left|\mathbf{r}-\mathbf{r}_{\mathbf{i}}\right|$ refers to any arbitrarily defined distance between observationally defined points.

The following points should be noted.

1. The form of Equation (1) which we use to define the density at a point is similar to the density estimate in the SPH simulations (e.g., Hernquist \& Katz 1989), with the exception of the selection function $\phi$. The density can be evaluated at a position of a galaxy (the so-called scatter approach, e.g., Hernquist \& Katz 1989), or at any chosen point in space (the so-called gather approach, e.g., Hernquist \& Katz 1989). The points can be of course chosen such that they form a regularly spaced grid.

2. The summation in Equation (1) goes over those galaxies in the sample that have been chosen to define the density field, which we refer to as tracer galaxies. These tracer galaxies might be all galaxies detected in the survey (which is commonly flux limited) or only a subsample of those satisfying some selection criteria, e.g., a criterion that is as much as possible independent of redshift, so as to form a "volume-limited sample."

3. The function $W\left(\left|\mathbf{r}-\mathbf{r}_{\mathbf{i}}\right| ; R\right)$ is the kernel used to weight the tracer galaxies, which is a spatial smoothing function (e.g., a top-hat or a Gaussian function) and $R$ is the smoothing length. The $W$ function is typically chosen such that it weights tracer galaxies depending on their distance $\left|\mathbf{r}-\mathbf{r}_{\mathbf{i}}\right|$ from the position where the density is being reconstructed. The distance between two points can be defined in various ways. In most applications the smoothing function $W$ shows some symmetry with respect to the reconstruction point. Commonly, it is of fixed shape and normalized to unity, i.e., $\int W d V=1$.

4. The smoothing length $R$ defines the aperture within which the environment is measured. To obtain a well-defined value at every point, the field needs to be filtered over a large 
aperture, such that the shot-noise effects are suppressed. However, all properties of the density field on scales smaller than the smoothing filter $R$ will be smoothed away. The smoothing length $R$ in a given reconstruction procedure can be defined to be of fixed or adaptive size. The fixed scale is then the same at every point and should be chosen such that there are enough tracer galaxies within the smoothing kernel to be able to reliably reconstruct the density at a given point. Unfortunately, in order to keep the number of galaxies in low density regions reasonably large to obtain statistically meaningful results, the smoothing length $R$ is then required to be rather large. The statistical fluctuations of the measured density will depend on the number of tracer galaxies within the smoothing aperture, and will be statistically more accurate in the regions with higher density. On the other hand, the adaptive scale varies over the volume in which the density field is being reconstructed. It is given by the density of the neighboring tracer galaxies, typically defined by the distance from the point of the density reconstruction (which can be a galaxy or any point in space) to the fixed $N$ th nearest neighbor. The use of an adaptive scale leads to a larger dynamical range and higher spatial resolution (in the dense regions) with respect to the fixed scale and the purely Poisson noise can also be made constant for a given population of tracer galaxies.

5. $m_{i}$ is the astrophysical weight ("mass") of the tracer galaxy. In the reconstruction of galaxy density fields it usually has a value of unity for each tracer galaxy, producing a "numberdensity." However, $m_{i}$ can be any measured property of the tracer galaxies (e.g., stellar mass). In the ideal case, it would probably be the total mass of a tracer galaxy. The resulting "mass" weighted density is supposed to be related to the underlying matter density via the bias $b$ factor. However, the bias can be a nonlinear and stochastic function, depending on the scale, redshift, and type of the tracer galaxies.

6. The reconstructed density field should be the density field based on the total population of chosen tracer galaxies in the observed volume. The function $\phi$ may be introduced to correct for the fact that in reality only a fraction of the full population of tracer galaxies is at our disposal when reconstructing the density field. The function $\phi$ should account for the observational restrictions such as the nonuniform sampling over the observed area, a radial selection function or the redshift success rate of the observed sources in the spectroscopic surveys. All of these constraints may depend on the intrinsic properties of galaxies (e.g., the luminosity or the morphological type) and as a result it may be very challenging to model all possible dependences into the $\phi$ function.

Unavoidably, the properties of the reconstructed density field will depend on the adopted method. The choice of a particular reconstruction method, given by the exact functional forms of $W\left(\left|\mathbf{r}-\mathbf{r}_{\mathbf{i}}\right| ; R\right), \phi\left(\mathbf{r}_{\mathbf{i}}\right)$, and $m_{i}$, and the choice of the tracer galaxies, is guided primarily by the scientific goals, but it will always contain some level of user-specified arbitrariness. In particular, one has to optimize between the scale at which the environment is measured and the error of the environment reconstructed on that scale, arising because we are using discrete tracers. While it may be desirable to measure environments on a very small scale, the statistical errors for the smallest scales are also the largest (e.g., Blanton et al. 2003; see also our results on the mock catalogs in Section 5). For some interpretations of the scientific results it may be desirable to use a fixed scale to define the environment, because the adaptive scales are smaller in the denser regions and larger in empty regions. However, the adaptive scales prove to be superior over the fixed scales in preserving the complex morphology of the density field (e.g., Park et al. 2007).

One limitation of the outlined scheme is its lack of sensitivity to the geometry of the mass distribution: the used kernels are commonly of fixed shape and isotropic. Even smoothing with the adaptive scale will smear out the smallest structures, particularly those of anisotropic shapes (e.g., Romano-Díaz \& van de Weygaert 2007). Shapiro et al. (1996) and Owen et al. (1998) introduced in simulations an elliptical kernel with axis ratio dependent on the geometrical distribution of particles.

The VTFE and DTFE are other methods to reconstruct the continuous density fields (Bernardeau \& van de Weygaert 1996; Schaap \& van de Weygaert 2000; Schaap 2007). Both VTFE and DTFE are fully adaptive and volume covering methods based on the Voronoi and Delaunay tessellation of the point sample, respectively, which divide space into a space-filling network of polyhedral cells (Voronoi tessellation, Dirichlet 1850; Voronoi 1908) or mutually disjunct tetrahedral cells in three dimensions (Delaunay tessellation, Delone 1934) according to the local density and geometry of the sampling points. Therefore, the VTFE and DTFE methods have the advantage over the methods which use kernels of fixed size to better recover the anisotropic structures in density fields, such as filaments and walls.

However, direct applications of the VTFE or DTFE to reconstruct the density field in the current $z \sim 1$ and higher redshift surveys also have some drawbacks. First, the spectroscopic surveys at these redshifts have usually a small angular size on the sky $\left(1-2 \mathrm{deg}^{2}\right)$, and with the current number density of the tracer galaxies the volumes of the Voronoi and Delaunay cells will be greatly affected at the edges. Cooper et al. (2005) used the Voronoi tessellation to estimate the local density of galaxies in the DEEP2-like mock catalogs. They conclude that more than $45 \%$ of the sample galaxies are affected by the edges when using the Voronoi tessellation to reconstruct densities around galaxies, compared with $\sim 15 \%$ when using a cylindrical kernel with $R=1 h^{-1} \mathrm{Mpc}$. Second, in $z \sim 1$ spectroscopic surveys the fraction of galaxies with a high quality spectrum, which leads to a reliable measure of a redshift used to delineate the density field, often is not greater than $\sim 50 \%$. It has not been investigated which effects this sampling fraction will have on an asymmetric density estimator such as DTFE. Moreover, it is not clear how to deal with probabilistic objects in the VTFE or DTFE (see van Breukelen et al. 2006, who use a Monte Carlo approach to sample the photometric redshift probability function of galaxies in the Voronoi tessellation method). The DTFE patterns also contain some artifacts, the most prominent one being the triangular imprint of the smoothing kernel (Schaap 2007; Romano-Díaz \& van de Weygaert 2007).

For some scientific applications, such as biasing, the density field needs to be reconstructed on a fixed scale. In a flux-limited survey, the mean separation between galaxies will increase with the redshift for a given smoothing scale and increase the shot noise. Even a given population of galaxies will be characterized by some average inter-galaxy separation, and therefore with any given galaxy population the density field on scales smaller than this separation will be dominated by the shot noise. For fixed apertures, the Wiener filtering technique can be used to deconvolve the noise from the reconstructed density field (e.g., Lahav et al. 1994; Hoffman 1994; Zaroubi et al. 1995). This 
technique is based on the minimum variance reconstruction of the density field, which requires the noise model and power spectrum of the density field to be known a priori.

It is common to express the resulting measurement of density as a dimensionless density contrast $\delta(\mathbf{r})$ defined as

$$
\delta(\mathbf{r})=\frac{\rho(\mathbf{r})-\rho_{m}(z)}{\rho_{m}(z)},
$$

where $\rho_{m}(z)$ is the mean density at a given redshift. In most of the applications $\rho_{m}(z)$ is evaluated as a volume average, but it can be estimated also as an average over galaxies (e.g., Cooper et al. 2007). Although we generally refer to $\delta$ also as "overdensity," we will characterize regions with $\delta>0$ as being overdense, and regions with $\delta<0$ as being underdense.

\section{ZADE METHODOLOGY}

The photometric redshift technique enables us to obtain redshifts of large number of galaxies in relatively modest amount of observing time with respect to spectroscopic surveys. The obtained photometric redshifts can be used for a variety of applications, such as luminosity and mass functions. Clearly, spectroscopic redshifts offer a major improvement over even high quality photometric redshifts. For instance, Cooper et al. (2005) find that for the density field reconstruction, if only the photometric redshifts are used, even with uncertainties as small as 0.02 , the reconstructed environment in the line-of-sight direction is smeared out on small scales. Only for uncertainties of photometric redshifts smaller than 0.005 and when measuring densities in projection can photometric redshift surveys become comparable to spectroscopic surveys in terms of environment reconstruction (Cooper et al. 2005). By including a properly devised background correction, the projected density estimator based on photometric redshifts becomes substantially unbiased, even though with a large scatter. This background corrected estimator is robust for projected densities larger than 10 galaxies per $\left(h^{-1} \mathrm{Mpc}\right)^{2}$ (Guzzo et al. 2007). However, the spectroscopic surveys with redshift uncertainties smaller than the velocity dispersion of the small group (i.e., below 200-250 $\mathrm{km} \mathrm{s}^{-1}$ ) are the preferred source of data with redshift of sufficient precision to reconstruct the small-scale environments of galaxies.

To reconstruct the density field on the given scale and with acceptable error one needs to balance between two requirements: precise spectroscopic redshifts to accurately trace the overdense and underdense regions, but as large number of objects as possible to lower the noise and enable reconstruction of the density on the smaller scales.

We have developed a new algorithm (ZADE) that brings together the accuracy of spectroscopic redshifts with the number statistics of photometric redshifts in order to broaden the scale of the reliable reconstructed environments. Motivated by the literature results of the importance of the scales at which the density is measured, our main effort has been made to reliably reconstruct the small-scale environments. The algorithm modifies the individual redshift probability distributions $P(z)$ that are output by a photometric redshift code (e.g., ZEBRA) based on the spectroscopic redshifts of galaxies located nearby on the sky.

Our motivation comes from the well-known observational evidence that galaxies are highly correlated on scales up to $10 h^{-1}$ Mpc. Large redshift surveys of galaxies, 2dFGRS and SDSS, are the $z \sim 0$ benchmarks for the precise measurements of the clustering signal (e.g., Norberg et al. 2001, 2002; Zehavi et al. 2005). DEEP2, VVDS, and now zCOSMOS confirm that galaxies are highly correlated up to $z \sim 1.5$ (e.g., Coil et al. 2006, 2008; de la Torre et al. 2007; Meneux et al. 2008, 2009). For example, Coil et al. (2006) find that the clustering scale length, $r_{0}$, is $3.69 \pm 0.14 h^{-1} \mathrm{Mpc}$ for the $M_{B}<19.8$ (using $h=0.7$ ) sample of galaxies in $0.75<z<1$ and $4.43 \pm 0.14$ $h^{-1} \mathrm{Mpc}$ for the $M_{B}<-21.3$ (using $h=0.7$ ) sample in $0.75<z<1.2$. At even higher redshifts, a high correlation signal has been measured for galaxies of a particular class, such as Lyman break galaxies (e.g., Lee et al. 2006) and Ly $\alpha$ emitters (e.g., Ouchi et al. 2003; Kovač et al. 2007). For example, the correlation length of Ly $\alpha$ emitters at $z \sim 4.5$ is $r_{0}=4.61 \pm 0.6$ $h^{-1} \mathrm{Mpc}$ (Kovač et al. 2007). Therefore, at least up to redshifts probed by the zCOSMOS-bright survey, any galaxy that has only a photometric redshift probability function $P(z)$, is much more likely to lie at some redshifts than at others, depending on the accurately determined spectroscopic redshifts of other galaxies that lie near to the same line of sight.

Our approach is purely empirical, and it can be summarized in the following way. The ZADE algorithm modifies the initial $P(z)$ that is the output of the photometric redshift program, by counting the number of objects with spectroscopic redshift within some radius $\left(R_{\mathrm{ZADE}}\right)$ as a function of $z$ along the line of sight, to yield a modified probability $P_{\mathrm{ZADE}}(z)$ :

$$
P_{\mathrm{ZADE}}(z)=\frac{N\left(\leqslant R_{\mathrm{ZADE}}, z\right) \times P(z)}{\int N\left(\leqslant R_{\mathrm{ZADE}}, z\right) \times P(z) d z} .
$$

In Figure 1, we show two examples of the ZADE approach to modify the initial $P(z)$, obtained using the ZEBRA code (Feldmann et al. 2006). For the computing purposes, we discretize the ZADE-modified $P(z)$ of every galaxy in $\Delta z=$ 0.002. At the current state of the uncertainty in the photometric redshifts, there are typically a few structures within the $P(z)$ along a given line of sight (effectively distribution of galaxies within $R_{\mathrm{ZADE}}$ ), so the ZADE approach is only statistical.

The ZADE approach to the density field reconstruction has been extensively tested on mock catalogs. We tested our method using five different fixed $R_{\mathrm{ZADE}}$ values: $1,2.5,5,7.5$, and $10 h^{-1} \mathrm{Mpc}$, and we have also experimented with the adaptive $R_{\text {ZADE }}$ values scaled to $\rho_{m}(z)$. We show in Figure 1 the effect of the modification of $P(z)$ for three values of $R_{\mathrm{ZADE}}(1,5$, and $\left.10 h^{-1} \mathrm{Mpc}\right)$. The value $R_{\mathrm{ZADE}}=5 h^{-1} \mathrm{Mpc}$ is chosen as an optimal ZADE radius to modify the photometric probability functions in order to reconstruct the density field without any systematics. We will justify our choice of $R_{\mathrm{ZADE}}$ in Section 5 , where we present some of the results from the tests on the mock catalogs.

Moreover, the undertaken tests led us to conclude that the use of the combination of objects with the spectroscopic and ZADEmodified photometric redshifts reconstructs the overdensity field at a given scale with smaller errors than the traditional approach of using only the spectroscopic sample of objects weighted with some $\phi\left(\mathbf{r}_{\mathbf{i}}\right)$ function in Equation (1) (which in our tests accounts for a nonuniform R.A.-decl. sampling). Therefore, this enables us to use smaller smoothing scales in the reconstruction process, and thereby broadens the dynamical range of the reconstructed environments.

For every density reconstruction within the zCOSMOS survey we use this combination of galaxies with spectroscopic and ZADE modified photometric redshifts. The summation in Equation (1) is therefore over all galaxies of a given population in the survey volume (and not only over a fraction of galaxies with reliable spectroscopic redshifts) equivalent to setting 

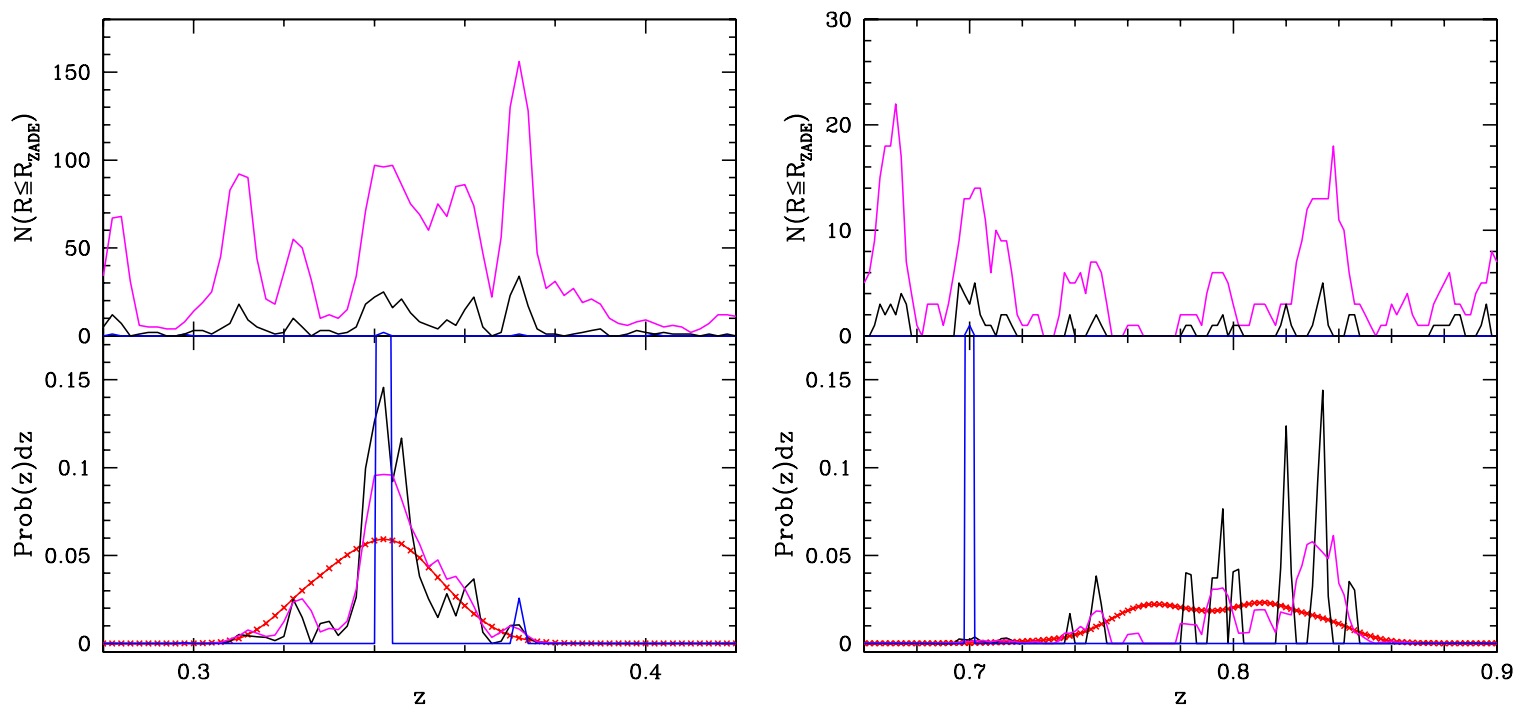

Figure 1. ZADE approach to modify the initial $P(z)$, discretized in $\Delta z=0.002$. The photometric redshift probability functions are presented in the lower panels. The $P(z)$ output from ZEBRA is presented in red and the ZADE-modified $P_{\mathrm{ZADE}}(z)$ are presented in blue $\left(\mathrm{R}_{\mathrm{ZADE}} \leqslant 1 h^{-1} \mathrm{Mpc}\right)$, black $\left(\mathrm{R}_{\mathrm{ZADE}} \leqslant 5 h^{-1} \mathrm{Mpc}\right)$, and magenta ( $\left.\mathrm{R}_{\mathrm{ZADE}} \leqslant 10 \mathrm{~h}^{-1} \mathrm{Mpc}\right)$. The number counts of objects with spectroscopic redshifts within $R_{\mathrm{ZADE}}$ radii at a given $z$ are presented in the upper panels, using the same color coding as in the lower panels. The left panel is for an object with $I_{\mathrm{AB}}=21.94 \mathrm{mag}$, the right panel is for an object with $I_{\mathrm{AB}}=22.38$ mag. Note that the number of objects with spectroscopic redshift within $R_{\mathrm{ZADE}} \leqslant 1 h^{-1} \mathrm{Mpc}$ is very small, and therefore $N\left(R \leqslant R_{\mathrm{ZADE}}=1 h^{-1} \mathrm{Mpc}\right)$ is zero at almost all $z$ (upper panels). See the text for more details.

$\phi\left(\mathbf{r}_{\mathbf{i}}\right)=1$ for every tracer galaxy. This is one of the major advantages of using galaxies with both spectroscopic and photometric redshifts, because in practice it is impossible to model the $\phi\left(\mathbf{r}_{\mathbf{i}}\right)$ function in such a way to take into account all the complex selection effects of most spectroscopic surveys. In Equation (1), galaxies with only photometric redshift will be counted with their ZADE-modified $P_{\mathrm{ZADE}}(z)$ according to a smoothing filter $W$.

\section{RECONSTRUCTING THE DENSITY FIELD WITHIN THE zCOSMOS VOLUME}

\section{1. zCOSMOS Survey}

zCOSMOS (Lilly et al. 2007, 2009) is a redshift survey undertaken in the $1.7 \mathrm{deg}^{2}$ COSMOS field (Scoville et al. 2007a). The observations are carried out with the VLT using VIMOS, a multi-slit spectrograph. In zCOSMOS-bright a magnitude selection $I_{\mathrm{AB}}<22.5$ has been applied to select galaxies in the redshift range up to $\sim 1.4$. The final sampling rate of the zCOSMOSbright survey should be a uniform $60 \%-70 \%$. This paper is based on the first 10,000 spectra, which have a rather nonuniform sampling pattern, $30 \%$ on average (see the figure with the 10k zCOSMOS sampling in Lilly et al. 2009). This sample of all spectra yields to the so-called "10k sample" of galaxies with only secure redshifts.

Thanks to the high-quality multi-wavelength photometry of the COSMOS survey (see Taniguchi et al. 2007; Capak et al. 2007), practically all galaxies in the zCOSMOS field can also have a photometric redshift. We employ the ZEBRA code in the maximum-likelihood mode (Feldmann et al. 2006) to calculate the full PDFs $P(z)$ and photometric redshifts (the maximum likelihood of $P(z)$ ) of galaxies in the COSMOS field, using typically 10 COSMOS broadbands. The uncertainty in the photometric redshifts that we use in this paper is $\sigma_{z}=0.023(1+z)$ at $I_{\mathrm{AB}}<22.5$. The accuracy in photometric redshifts will further improve, when new photometric data become available (e.g., Ilbert et al. 2009; Salvato et al. 2009).
In our analysis, we complement the "10k sample" of galaxies with $\sim 25,000 I_{\mathrm{AB}}<22.5$ galaxies which do not have spectroscopic redshifts yet, but for which photometric redshifts are available. We refer to this composite sample as the " $10 \mathrm{k}+30 \mathrm{k}$ sample." We additionally refer to the "40k sample" as the set of all galaxies with $I_{\mathrm{AB}}<22.5$ in the area of the zCOSMOS survey. In the analysis below, we will frequently make use of mock catalogs (based on Kitzbichler \& White 2007) for various tests, in which all galaxies in the "40k sample" have accurately known redshifts.

We use rest-frame absolute magnitudes of galaxies obtained using the ZEBRA code as the best-fit template normalized to each galaxy photometry and best available redshift (spectroscopic or photometric; P. Oesch et al. 2010, in preparation). For the galaxies with only photometric redshift, a prior imposes $M_{B}$ to be in the range between -13 and -24 mag.

Stellar masses $M_{*}$ are obtained from fitting stellar population synthesis models to the multicolor spectral energy distribution (SED) of the observed magnitudes (see Bolzonella et al. 2009; Pozzetti et al. 2009). In this work, we use stellar masses calculated using the Bruzual \& Charlot (2003) libraries, with the Chabrier initial mass function (Chabrier 2003). The star formation history (SFH) is assumed to decrease exponentially with a time scale $\tau$, where $0.1 \mathrm{Gyr}<\tau<30 \mathrm{Gyr}$. The Calzetti et al. (2000) extinction law was used with $0<A_{V}<3$ and solar metallicities. The final stellar mass is obtained by integrating the SFH over the galaxy age and subtracting from it the so-called return fraction, which is the mass of gas processed by stars and returned to the interstellar medium during their evolution. The stellar masses are calculated using the best available redshifts for each galaxy.

\subsection{The Choices to Measure the Density Field with zCOSMOS}

Given the diversity of the scientific applications of the density field reviewed above, we have produced a large variety of environment measures that are appropriate for different applications. In the following text, we discuss the particular choices used for the measurement of the zCOSMOS density 
field. For practical purposes, we use the symbol $\rho$ for all types of densities.

\subsubsection{Tracer Galaxies: Flux Limited or Volume Limited?}

For the ZADE zCOSMOS overdensity reconstructions, all galaxies with $I_{\mathrm{AB}}<22.5$ are considered as possible tracer galaxies without taking into account the quality of their measured redshift (spectroscopic or photometric) within the zCOSMOS region. Galaxies with high confidence redshift class (flags starting with 3, 4, and 9, and classes 1.5, 2.4, and 2.5) and the broad-line emitters with high confidence redshift class (flags obtained by adding "10+" to the above classes) are counted as one object at the spectroscopic redshift (see Lilly et al. 2007, 2009 for the flag definition). The secondary objects with the same redshift confidence class were also used (flags obtained by adding "20+" to the above classes). The selected sample includes objects with highly secure redshifts (redshifts for the all flag 2 objects have security above $92 \%$ based on the spectroscopic verification) and those objects whose spectroscopic redshifts are consistent with their photometric redshift within $0.08(1+z)$. The sample includes also the objects with a single line measured, which could be only $\mathrm{H} \alpha$ or [O II $] 3727$ (flag 9) and whose redshift is set to the redshift consistent with the photometric redshift. There are only five objects with flag 9 in the relevant redshift range for which the photometric redshift is not available. Repeated observations exist for about $5 \%$ of the $10 \mathrm{k}$ sample. These are used to obtain the spectroscopic verification rate and velocity accuracy. Using the probabilistic approach, it has been estimated that the redshifts of the selected classes of galaxies are individually $99 \%$ reliable (Lilly et al. 2009). This set of objects makes about $88 \%$ of the spectroscopic sample, or $95 \%$ at $0.5<z<0.8$. Up to $z \sim 1$, we do not detect a significant dependence of the assigned redshift class on the type of a galaxy. The velocity accuracy is estimated to be $\sigma_{v}=$ $110 \mathrm{~km} \mathrm{~s}^{-1}$ or $\sigma_{z}=0.00036(1+z)$ (Lilly et al. 2009).

For the rest of galaxies within the zCOSMOS survey limits we use the ZADE-modified ZEBRA photometric redshift output. For the few galaxies without a photometric redshift estimate, we use the ZADE-modified $N(z)$ distribution of all galaxies in the fields as initial $P(z)$, normalized to unity within the redshift interval $0<z<1.4$ (only a negligible fraction of galaxies with high confidence redshift class has a redshift $z>1.4$ ). For the few QSOs with a low confidence redshift we use the same ZADE-modified $N(z)$ distribution to be their probability function, but normalized to $f_{\mathrm{QSO}}$, which is the fraction of the broad-line objects with high-confidence class below $z=1.4$ in the sample of the broad-line objects with high-confidence class detected at all redshifts. For the 10k zCOSMOS sample, $f_{\mathrm{QSO}}=0.43$.

In summary, we use spectroscopic redshift information of galaxies with a spectroscopic redshift reliability of $99 \%$, combining these with the ZADE-modified photometric redshifts for the remaining galaxies that are either without a secure redshift or that have not yet been observed spectroscopically.

We construct three different samples of tracer galaxies to measure the zCOSMOS over-density field. For the "flux-limited sample," all galaxies within the zCOSMOS region with $I_{\mathrm{AB}}<$ 22.5 are used as tracer galaxies. In total, there are 33,211 such galaxies, of which 8341 have a reliable spectroscopic redshift. In addition, we define two volume-limited samples of tracer galaxies, which should ideally include the same galaxies at every redshift. Understanding of galaxy evolution does not run deep enough to provide a recipe of galaxy evolution which includes all physical processes for every single galaxy (e.g., star formation episodes of various activity and merging of galaxies). Therefore, to constrain the volume-limited samples, we just take into account a passive evolution of galaxies of 1 mag per unit redshift interval, which should on average account for most of the evolution for the majority of galaxies. The two volume samples satisfy the criteria $M_{B} \leqslant(-19.3-z)$ and $z \leqslant 0.7$, and $M_{B} \leqslant(-20.5-z)$ and $0.4<z \leqslant 1.0$. To include galaxies with only photometric redshifts in the volume-limited samples of tracer galaxies we use their maximum likelihood redshift and the $M_{B}$ magnitude at the same redshift. However, in the density field reconstruction, we use the $P_{\mathrm{ZADE}}(z)$ distribution of these galaxies. Galaxies without photometric redshift or $M_{B}$ are not used to construct the volume-limited samples of tracer galaxies. The defined volume-limited samples are complete samples, and they are selected to be the largest possible samples at these redshifts.

\subsubsection{Filter $W$ and Smoothing Length $R$}

Starting from the work of Dressler (1980), when studying the galaxy properties as a function of their environment, it is common to project the tracer galaxies within some velocity interval, and then estimate the surface density. The main advantage of using the projected distances instead of the full three-dimensional distances is to minimize the effects of peculiar velocities. The aperture, perpendicular to $z$, within which the environment is measured can be of fixed or adaptive size. Dressler (1980) for example used the projected distance to the 10th nearest neighbors to define a rectangular aperture.

Following the adopted formalism to describe the density field (Equation (1)), the filter $W$ in this approach is

$$
\begin{aligned}
& W\left(\left|\mathbf{r}-\mathbf{r}_{\mathrm{i}}\right| ; D_{p}\right) \\
& =\left\{\begin{array}{c}
\frac{1}{\pi D_{p}^{2}} P_{i, \mathrm{ZADE}}(z \pm \delta z) \\
\text { if }\left|r_{p}-r_{i, p}\right| \leqslant D_{p},\left|z_{i}-z\right| \leqslant \delta z\left(\delta z=1000 \mathrm{~km} \mathrm{~s}^{-1}\right) \\
0 \quad \text { otherwise. }
\end{array}\right.
\end{aligned}
$$

This smoothing kernel is of cylindrical shape, with a radius of $D_{p}$ in the R.A.-decl. plane and a length of $\pm \delta z$ along the line of sight. The distance $\left|r_{p}-r_{p, i}\right|$ is measured between the point where we are reconstructing the density field and the individual tracer galaxy projected to the redshift of that point. The $P_{i, \mathrm{ZADE}}(z \pm \delta z)$ is the integrated probability of the tracer galaxy to be within the $z \pm \delta z$ interval. For galaxies with spectroscopic redshift within this interval $P_{i, \mathrm{ZADE}}(z \pm \delta z)=1$. We use the interval $\delta z$ given by distance of $1000 \mathrm{~km} \mathrm{~s}^{-1}$ to the redshift $z$ of the point where the density field is measured. Varying $\delta z$ by $\pm 500 \mathrm{~km} \mathrm{~s}^{-1}$ around the chosen value of $1000 \mathrm{~km} \mathrm{~s}^{-1}$ does not make a significant difference.

Using the spatial filter $W$ given in Equation (4), we measure the density field at the positions of galaxies and on a regularly spaced grid. For the former, we include the central galaxy into the counts of objects if this galaxy passes the criterion to be a tracer galaxy. Using the ZADE approach, a tracer galaxy can have either spectroscopic or photometric redshift.

We use both fixed and adaptive apertures to estimate the density only at the positions of galaxies with high quality spectroscopic redshifts. We define the adaptive aperture as the projected distance to the $N$ th nearest neighbor $D_{N}$, excluding the central galaxy from the nearest neighbors search, but including it again when estimating the density. For the measurement on a grid we use the adaptive projected scale $D_{p}=D_{N}$ defined by the 

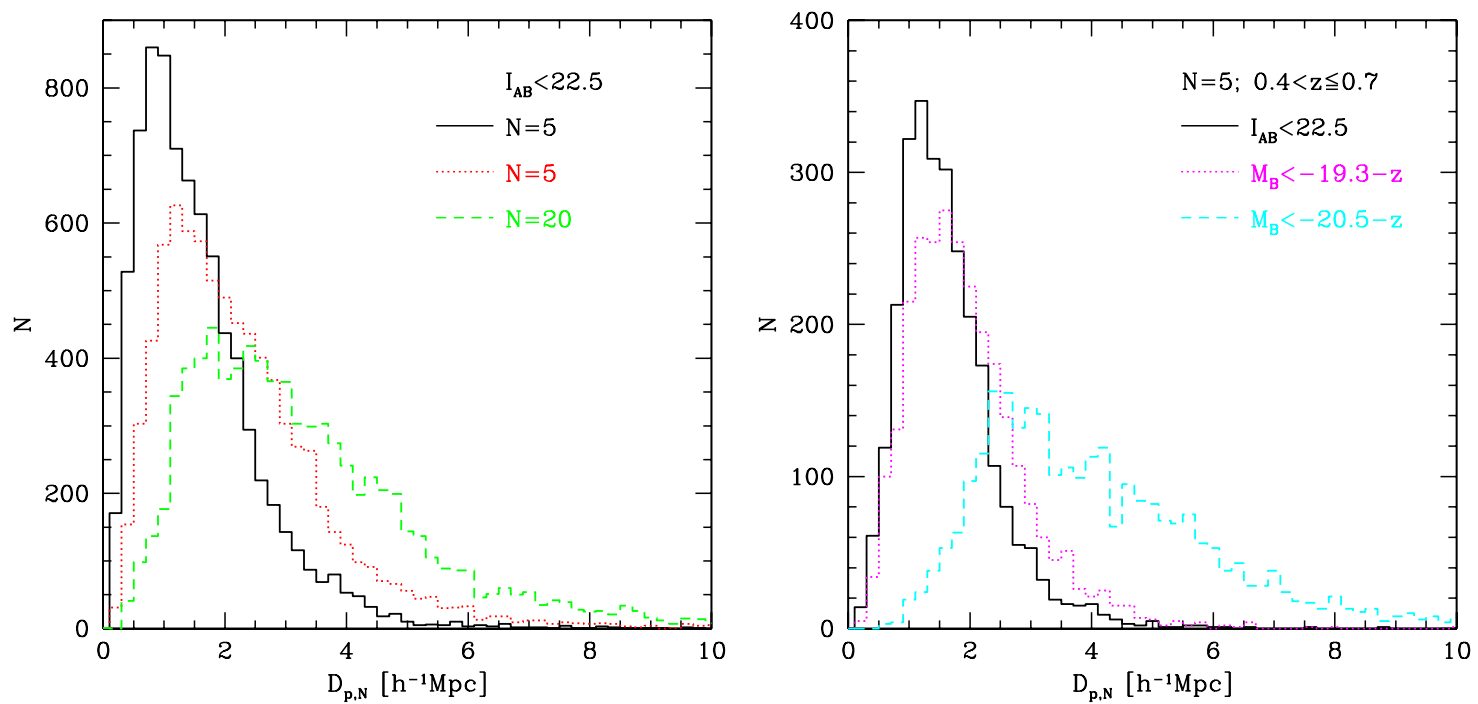

Figure 2. Distribution of the aperture sizes defined by the $N$ th nearest neighbor centered at the positions of the zCOSMOS galaxies with spectroscopic redshift of high confidence. Tracer galaxies are projected within $\pm 1000 \mathrm{~km} \mathrm{~s}^{-1}$ of the galaxy redshift.

distance to the $N$ th nearest neighbor. Given that we are working also with the probability functions (and therefore fractional objects), in practice, we measure the adaptive aperture defined by the distance to the $N$ th nearest neighbor as the minimum distance for which $\sum_{i} P_{i, \mathrm{ZADE}}(z \pm \delta z) \geqslant N$. This is valid for the estimates of the density centered on both galaxies and on grid points. Based on tests on the mock catalogs, some of which will be discussed in Section 5, we find that the smallest fixed aperture that can be used for the density reconstruction centered at the zCOSMOS galaxies is $3 \mathrm{Mpc} h^{-1}$, while the smallest useful adaptive aperture is defined by the fifth nearest neighbor. We present the distribution of the aperture sizes defined by the fifth, 10th, and 20th nearest neighbors when using the fluxlimited tracers in the left panel of Figure 2. The distribution of the aperture sizes defined by the fifth nearest neighbor in the $I_{\mathrm{AB}}<22.5, M_{B}<(-19.3-z)$, and $M_{B}<(-20.5-z)$ samples in the $0.4<z \leqslant 0.7$ bin in which we are complete for all subsamples of tracer galaxies is shown in right panel of Figure 2. When using the volume-limited samples of tracer galaxies, the distance to the $N$ th nearest neighbor is clearly equal or larger than the distance when using the flux-limited sample of tracer galaxies. In the redshift range $0.4<z \leqslant 0.7$, the adaptive apertures are smaller than $3 h^{-1} \mathrm{Mpc}$ for $94 \%, 88 \%$, and $62 \%$ galaxies for the flux limited and the $M_{B}<(-19.3-z)$ and $M_{B}<(-20.5-z)$ volume-limited samples of tracer galaxies, respectively. We will discuss the relation between the apertures and the reconstructed overdensity for the zCOSMOS galaxies in Section 6.1.

Some of the previous studies of the galaxy evolution as a function of environment are based on the density field reconstructed using the three-dimensional Gaussian filter (e.g., Cucciati et al. 2006). To enable a proper comparison with such results, we also use a Gaussian filter to reconstruct the density field on scale $R_{G}$ at the positions of zCOSMOS galaxies:

$$
\begin{aligned}
& W\left(\left|\mathbf{r}-\mathbf{r}_{\mathbf{i}}\right| ; R_{G}\right) \\
& = \begin{cases}\frac{1}{\left(2 \pi R_{G}^{2}\right)^{(3 / 2)}} P_{i, \operatorname{ZADE}}\left(z_{i}\right) \exp \left[-\frac{1}{2}\left(\frac{\mathbf{r}-\mathbf{r}_{\mathbf{i}}}{R_{G}}\right)^{2}\right] & \text { if }\left|\mathbf{r}-\mathbf{r}_{\mathbf{i}}\right| \leqslant R_{G} \\
0 & \text { otherwise. }\end{cases}
\end{aligned}
$$

The $\left|\mathbf{r}-\mathbf{r}_{\mathbf{i}}\right|$ is a three-dimensional separation between positions of a tracer galaxy and a galaxy where the density is being evaluated. If the central galaxy belongs to the sample of tracer galaxies used, it is also included in the counts.

For the biasing analysis (Kovač et al. 2009), we use a different scheme to reconstruct the overdensity field. We want to compare the overdensity field traced by galaxies to the underlying matter overdensity, and therefore we need the overdensity field estimated on the same scale as the mass overdensity as available in the literature. Thus, we use the spherical top-hat filter to reconstruct the density field of galaxies:

$$
W\left(\left|\mathbf{r}-\mathbf{r}_{\mathbf{i}}\right| ; R_{\mathrm{TH}}\right)= \begin{cases}\frac{1}{\frac{4}{3} \pi R_{\mathrm{TH}}^{3}} P_{i, \mathrm{ZADE}}\left(z_{i}\right) & \text { if }\left|\mathbf{r}-\mathbf{r}_{\mathbf{i}}\right| \leqslant R_{\mathrm{TH}} \\ 0 & \text { otherwise, }\end{cases}
$$

where $R_{\mathrm{TH}}$ is the smoothing scale of the top-hat filter. Here, $\left|\mathbf{r}-\mathbf{r}_{\mathbf{i}}\right|$ is a three-dimensional distance between a tracer galaxy and a point where the density is being estimated. An obvious shortcoming of this reconstruction is that it is affected by peculiar motions. We include this into consideration for the biasing analysis.

When the smoothing length $R$ of a filter $W$ is defined using the projected distances (as in Equation (4)), we will denote the reconstructed overdensity with $\delta_{p}$. In the case when $R$ is defined using the three-dimensional distance (as in Equations (5) and (6)), we will denote the reconstructed overdensity with $\delta$.

\subsubsection{Weighting $m_{i}$ of Galaxies}

In addition to the number overdensity reconstruction, we also calculate the $B$-band luminosity and stellar mass $M_{*}$ weighted densities. Following Equation (1), these three types of reconstructed environment correspond to the cases of $m_{i}=1$, $m_{i}=L_{B}$, and $m_{i}=M_{*}$, respectively. We assume that the physical properties of galaxies are constant, i.e., they do not change along redshift with $P_{\mathrm{ZADE}}\left(z_{i}\right) . L_{B}$ and $M_{*}$ are estimated at the best available redshift for each galaxy.

Weighting each galaxy with its physical property will lead to a galaxy density field still mainly determined by the spatial distribution of galaxies, with some additional discrimination based on the properties of galaxies. For example, for the same number of galaxies in two identical apertures, the measured number density will obviously be the same, while weighting 
galaxies with their $L_{B}$ or $M_{*}$ will add a finer differentiation between the measured densities.

A shortcoming of the density estimate with $m_{i} \neq 1$ is that any relations between galaxy properties and environment can contain some degree of correlation between the studied galaxy property and the property of the galaxy used to weight the galaxy counts for the density reconstruction. This is particularly pertinent when including the central galaxy itself in the measurement of the environment. For example, the color-density relation will become (artificially) more significant when using $M_{*}$-weighted overdensities compared to the number overdensities (see Cucciati et al. 2009), and the differences between the stellar mass functions in the overdense and underdense regions will appear more pronounced (see Bolzonella et al. 2009). Nevertheless, $m_{i} \neq 1$ weighting can be useful in defining a physically better motivated density estimator.

\subsection{Estimating Mean Density}

To convert the density to an overdensity an estimate of the average density is required. Typically, galaxies in the survey itself are used to define the mean density, a value which is intended to be valid in the whole universe (at a given redshift). Therefore, one needs to assume that these surveys are a fair sample of the universe.

There are various ways to determine the mean density of a survey. One of the commonly used methods is smoothing the observed number distribution of the tracer galaxies in the redshift dimension, or fitting this distribution with some assumed functional form. Sometimes only the pure number density of objects in some redshift interval is used, but in this case a part of the true variations in the overdensity with redshift will be washed away. The other commonly used method is deriving mean densities of galaxies from the galaxy luminosity function, in which case the redshift evolution of the luminosity function needs to be modeled. We use here a non-parametric treatment of the luminosity function approach.

We use the full population of tracer galaxies from the zCOSMOS survey (selected from the $10 \mathrm{k}+30 \mathrm{k}$ sample) to define the mean volume density as a function of redshift. For each galaxy, we first calculate the maximum volume $V_{\max }$ in which this galaxy could be detected in the zCOSMOS survey, including for completeness an assumed passive evolution of $\Delta m=\Delta z$ mag. In the next step, the contribution of this galaxy to the density in each redshift bin, up to $z_{\max }$ is calculated as $\Delta V(z) / V_{\max }$, where $\Delta V(z)$ is the volume of the individual redshift bins. $z_{\max }$ is the maximum redshift up to which a galaxy of a given luminosity could be detected in a $I_{\mathrm{AB}}<22.5$ survey; $V_{\max }$ is the volume of the zCOSMOS survey up to redshift $z_{\max }$. This is carried out for every tracer galaxy and then the contribution of all galaxies is summed to obtain $N(z)$. We present the $N(z)$ distributions for the three samples of the zCOSMOS tracer galaxies in Figure 3. Similarly, one can calculate also the luminosity and stellar mass weighted number of objects by simply weighting $\Delta V(z) / V_{\max }$ contributions by $L_{B}$ and $M_{*}$, respectively.

As desired, the procedure outlined above introduces a smoothing in the very peaky observed distribution of objects, as it can be seen in Figure 2. With respect to a more standard smoothing on the observed $N(z)$ distribution, it has the advantage of not requiring any assumption on the smoothing length. The kernel in this smoothing procedure is of quadrilateral shape, where one side is defined by the distance between $z_{\min }$ and $z_{\max }$, and the vertical sides are given by $\Delta V(z) / V_{\max }$ at $z_{\min }$ and $z_{\max }$. The

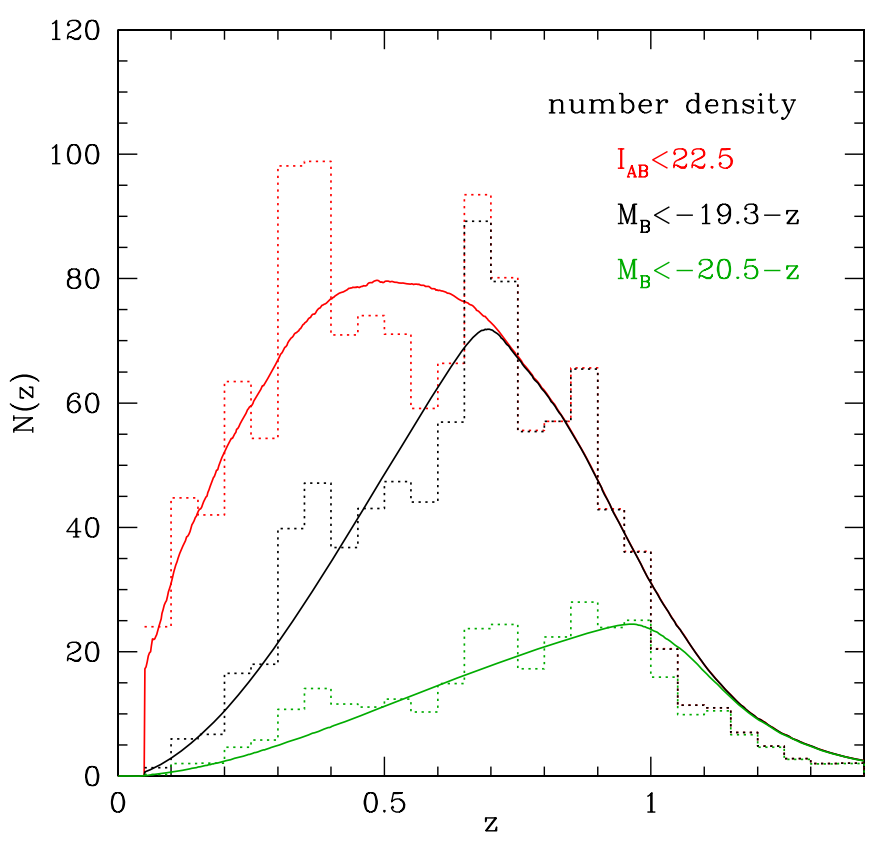

Figure 3. Redshift distribution of galaxies in the zCOSMOS area. The continuous lines correspond to the smoothed $N(z)$ distributions obtained by weighting galaxies according to their $\Delta V(z) / V_{\max }$ contribution in $\Delta z=0.002$ intervals as described in the text. The dotted lines correspond to the histogram distributions of tracer galaxies in the redshift bins of $\Delta z=0.05$ and they are divided by 25 to match the redshift bin of the smoothed $N(z)$. Different colors represent different samples of tracer galaxies, as indicated in the top right corner of the plot. The samples of galaxies with $M_{B}<(-19.3-z)$ and $M_{B}<(-20.5-z)$ are volume-limited up to $z<0.7$ and $z<1$, respectively.

remaining side of this kernel is curved, defined by $\Delta V(z) / V_{\max }$ in redshifts between $z_{\min }$ and $z_{\max }$. The exact shape of the kernel depends on $I_{\mathrm{AB}}$ of the individual galaxy. Due to the curved side of the kernel, a large number of galaxies at $z \sim 0.85$ produces a somewhat overestimated $N(z)$ above redshift of 1 . The produced $N(z)$ distributions using the spectroscopic redshifts or only maximum probability redshifts for the same group of objects are almost identical.

Finally, we obtain the mean density $\rho_{m}(z)$ by normalizing $N(z)$ or its weighted counterparts by the "volume" in which $N(z)$ is estimated. When the density is calculated using the three-dimensional distances, this is obtained dividing $N(z)$ by the volume of the zCOSMOS survey in a redshift bin centered at redshift $z$ of that galaxy (or a grid point). To obtain the surface mean density we first integrate $N(z)$ in the interval of \pm 1000 $\mathrm{km} \mathrm{s}^{-1}$ centered on the redshift of a galaxy or a grid point of interest to obtain the corresponding number of objects, which we afterward divide by the zCOSMOS area.

\section{ZADE IN THE DENSITY RECONSTRUCTION: AFFIRMATION ON MOCK CATALOGS}

\subsection{ZADE Reconstruction with respect to the Total Population of Tracer Galaxies}

We use mock catalogs to assess the performance of the density field estimator based on the combination of galaxies with the spectroscopic and ZADE-modified photometric redshifts when applied on the 10k zCOSMOS survey. The mock catalogs for the zCOSMOS survey are extracted from the lightconemock catalogs described in Kitzbichler \& White (2007). These lightcones are based on the dark matter $N$-body Millenium Simulation run (Springel et al. 2005) with $\Omega_{m}=0.25, \Omega_{b}=$ 
$0.045, h=0.73, \Omega_{\Lambda}=0.75, n=1$, and $\sigma_{8}=0.9$. The semianalytic galaxy modeling that is applied to the mock catalogs is described in Kitzbichler \& White (2007). Essentially, it follows the model of Croton et al. (2006) as updated by De Lucia \& Blaizot (2007). The only change is in the dust model, developed to better match the observations of the mass functions at higher redshifts (Kitzbichler \& White 2007). There are 24 independent mock catalogs of an area $1.4 \times 1.4$ and $z \lesssim 7$, with a flux limit $r \leqslant 26$ mag. We use 12 of these mocks, randomly chosen, to perform various tests.

The mocks are used in the following way. All mocks are cut to match the exact zCOSMOS area and redshift limits. We define the "parent" catalog (equivalent to the 40k catalog, $\left.I_{\mathrm{AB}}<22.5\right)$, in which we assume all tracer galaxies in the zCOSMOS volume have a high quality spectroscopic redshift, and the "observed" catalog, in which about 10,000 galaxies have spectroscopic redshifts and the rest of the tracer galaxies have only photometric redshifts (analogous to the $10 \mathrm{k}+30 \mathrm{kZADE}$ catalog in the real data). The distribution of objects with the spectroscopic redshifts in the observed catalogs is modeled (see Knobel et al. 2009) to resemble that of the true 10k zCOSMOS catalog (Lilly et al. 2009).

In the mock catalogs, we model the photometric redshift probability function $P(z)$ as a Gaussian of dispersion $\sigma_{z}$ depending on the selection $I_{\mathrm{AB}}$ magnitude. For galaxies with $I_{\mathrm{AB}}<22.5 \mathrm{mag}$ we use $\sigma=0.023(1+z)$ for the purpose of the simulations. Galaxies are randomly displaced from their true redshifts by an amount selected from this same distribution. We then apply the ZADE algorithm on those galaxies without reliable spectroscopic redshifts in the mock catalogs using different values of the $R_{\text {ZADE }}$ radii. We have done a simple check of the robustness of our results with respect to the functional form of the probability function by randomly assigning actual probability functions, obtained from ZEBRA for the real zCOSMOS galaxies, to the galaxies in the mock catalog. Our results did not change significantly. The shape of the ZADE-modified $P_{\mathrm{ZADE}}(z)$ is determined mostly by the neighboring galaxies and not by its initial form.

For the test on the mock catalogs, the (over)density field is calculated on a grid equally spaced in the angular units $\Delta \alpha=$ $\Delta \delta=2 \operatorname{arcmin}$ and in the redshift $\Delta z=0.002$. The density $\rho$ in the individual grid points is estimated using Equation (4), with the aperture defined by the distance to the $N$ th nearest neighbors $\mathrm{D}_{p, N}$. As we mention earlier, because we also work with "fractional" objects, in this reconstruction we count the number of objects until $\Sigma_{i} P_{i}(\Delta z) \geqslant N$.

To obtain overdensities, the $\rho$ estimate in the individual grid points is divided by $\rho_{m}(z)$. In this way, the reconstructed measure of environment is given in the units of $1+\delta_{p}=\rho / \rho_{m}$ (Equation (2)). For the individual mock catalogs, $\rho_{m}(z)$ is obtained from the smooth number distribution $N(z)$ of the full $40 \mathrm{k}$ sample of tracer galaxies. We obtain the smooth $N(z)$ distributions separately in each mock to closely resemble the uncertainties in $\rho_{m}(z)$ arising from the limited volume of the real survey.

We present here only the number overdensities, obtained using $m_{i}=1$ for all the tracer galaxies. The tracer galaxies are selected from the flux-limited sample of galaxies. In the following plots, the survey area is limited to the central $0.8 \times 0.8 \mathrm{deg}^{2}$ in right ascension and declination to minimize the edge effects.

Obviously, in the ZADE approach $R_{\mathrm{ZADE}}$ is a free parameter. In Figure 4, we present median values of differences between the $10 \mathrm{k}+30 \mathrm{kZADE}$ observed and the $40 \mathrm{k}$ parent overdensities at a given 10k+30kZADE observed overdensity, where
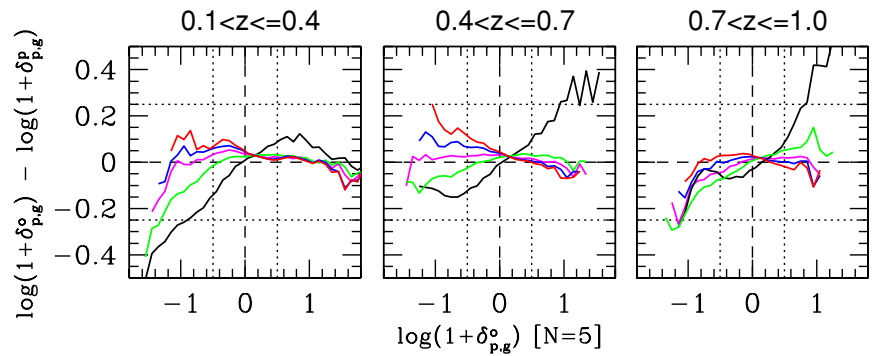

Figure 4. Comparison between the $10 \mathrm{k}+30 \mathrm{kZADE}$ observed and $40 \mathrm{k}$ parent overdensities at a given value of the observed overdensity for different $R_{\text {ZADE }}$ radii. The density is reconstructed on the projected apertures defined by the distance to the fifth nearest neighbor. The continuous lines represent medians for the measured difference between the overdensities reconstructed in the observed catalog, using the ZADE formalism, and in the parent catalog, at a given value of the observed overdensity. The black, green, magenta, blue, and red are medians for the ZADE reconstructions with $R_{\mathrm{ZADE}}$ taking value of $1,2.5,5,7.5$, and 10 $h^{-1} \mathrm{Mpc}$, respectively. The redshift intervals used for the analysis in this and similar plots are indicated on top of each plot. In this paper, we use $R_{\mathrm{ZADE}}=5$ $h^{-1} \mathrm{Mpc}$ which gives small bias at all $z$ and $\delta_{p}^{o}$. The lower indices "p" and " $\mathrm{g}$ " indicate that the overdensity has been reconstructed using the projected distances to define a smoothing length $R$ and has been reconstructed on the grid, respectively. The upper index refers to the type of the mock catalog; "p" stands for the parent and "o" for the observed catalog.

the $10 \mathrm{k}+30 \mathrm{kZADE}$ reconstruction has been carried out with different $R_{\text {ZADE }}$ radii. Based on the correlation function results (see Section 3 ), we have considered $R_{\mathrm{ZADE}}$ to be of the order of a few $h^{-1} \mathrm{Mpc}$. The results are presented for the fixed values of $R_{\text {ZADE }}$ of $1,2.5,5,7.5$, and 10 comoving $h^{-1} \mathrm{Mpc}$. The amount of the scatter of the values around the median at a given observed overdensity is similar for the different $R_{\text {ZADE }}$ radii, and we did not plot it for clarity. One can inspect Figure 6 to get the idea about the scatter in the presented relations. As visible in Figure 4, statistically, the 10k+30kZADE reconstruction does not show much systematic offset from the 40k reconstruction for $2.5 h^{-1} \mathrm{Mpc}<R_{\mathrm{ZADE}}<7.5 h^{-1} \mathrm{Mpc}$. Similar results have been obtained also when using $R_{\mathrm{ZADE}}=\rho_{m}(z)^{-1 / 3} \cdot R_{\mathrm{ZADE}}=$ $5 \mathrm{~h}^{-1} \mathrm{Mpc}$ is the radii for which the $10 \mathrm{k}+30 \mathrm{kZADE}$ overdensities show the least systematic offset from the 40k overdensities, and we therefore adopt $R_{\mathrm{ZADE}}=5 \mathrm{~h}^{-1} \mathrm{Mpc}$ for the all subsequent analyses, both on the mocks and the real data.

Figure 5 presents a comparison of the $10 \mathrm{k}+30 \mathrm{kZADE}$ observed and the 40k parent overdensities for the individual grid points, using the distance to the 10th nearest neighbor to obtain densities. The thick continuous curve represents the median for the $10 \mathrm{k}+30 \mathrm{kZADE}$ observed and the thick dashed curves correspond to the 25 th and 75 th percentiles for the $10 \mathrm{k}+30 \mathrm{kZADE}$ observed overdensities. Binning is done as a function of parent overdensity. Obviously, the ZADE method is not perfect-it slightly overestimates the density in the most underdense regions, and it underestimates the density in most overdense regions, no doubt because of residual smearing of the initial $P(z)$ for the objects with only photometric redshifts. Note, however, that the median relation between the observed and parent catalog is different if the binning is done along the parent or observed axis. Generally, the reconstructed overdensity reproduces the parent overdensity without a significant systematic effect over most of the range, within the errors. This is more clearly visible in Figure 6, where in all three panels we present the difference between the observed $10 \mathrm{k}+30 \mathrm{kZADE}$ and the parent $40 \mathrm{k}$ overdensities for the individual grid points, plotted as a function of the observed overdensity. The density field is reconstructed using three different adaptive apertures defined by the distance to 


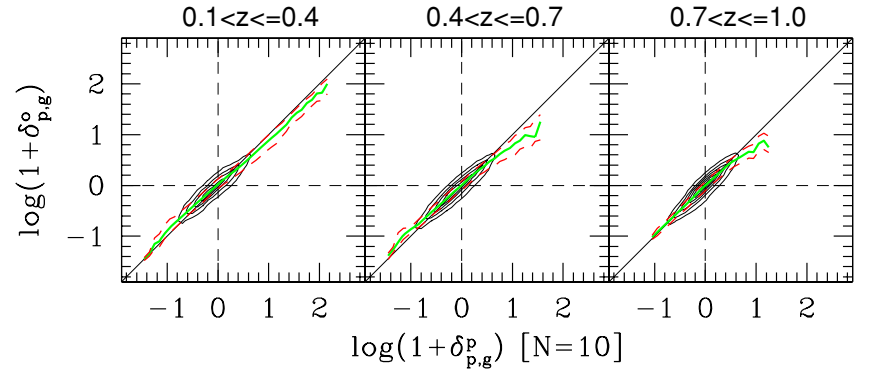

Figure 5. Comparison between the $10 \mathrm{k}+30 \mathrm{kZADE}$ observed and $40 \mathrm{k}$ parent overdensities. The contours are obtained by binning the overdensities in the individual grid points in the $\Delta \log \left(1+\delta_{p}\right)=0.1$ intervals with a step of 500. The thick continuous curve represents the median for the 10k+30kZADE observed, the thick dashed curves correspond to the 25th and 75th percentiles for the $10 \mathrm{k}+30 \mathrm{kZADE}$ observed. Binning is done along the parent overdensity axis. Overdensities are obtained by using the distance to the 10th nearest neighbor. Note that $50 \%$ of all points are contained between the dashed curves. The indices are defined in Figure 4.

(A color version of this figure is available in the online journal.)

the fifth, 10th, and 20th nearest neighbor. Note that due to the ZADE approach we can compare overdensities obtained in the apertures defined by the same $N$ (number of nearest neighbors) in the parent and observed catalogs since they both contain the same total number of objects.

Based on the difference between the 25 th and 75 th percentiles, the error (statistical scatter) in the reconstructed overdensities using the ZADE method is roughly $0.1-0.15$ dex in $1+\delta_{p}$. It is clear that the error on the reconstructed overdensity gets smaller when using a larger number of neighbors, i.e., the density measured at larger apertures. Depending on the scientific goal, one has to balance between the smoothing scale and the statistical error on the reconstructed overdensity. The presented statistical errors include only those uncertainties arising from the fact that we are dealing with a population of galaxies with very different qualities of their measured redshifts (i.e., 10k galaxies with spectroscopic redshifts and 30k galaxies with the ZADE modified photometric redshifts). We did not include any errors arising from the uncertainties in the spectroscopic redshift or in the measured physical property. Also, these errors do not account for any differences between the 40k overdensities and the true matter overdensities. We will discuss some of these additional errors in more detail in Section 5.3. If we redo the comparison using the full survey area, and not only the inner part, the median and lower and upper quartiles in Figures 5 and 6 remain almost unchanged. For example, in Figure 5 there is an indication of a larger underestimation in the most overdense regions.

Figures 5 and 6 are obtained by using one mock catalog. In Figure 7, we present the difference between the observed and parent overdensities as a function of the observed overdensities for all 12 mock catalogs. The errors (i.e., the 25th and 75th percentiles of the difference between the observed and parent overdensity) at a given observed overdensity are similar for all the individual mock catalogs (and we do not show them in Figure 7). The errors in Figure 6 can therefore be taken as a reference for the errors in the observed overdensities, when using this particular method of the reconstruction of the density field.

We have chosen to present the errors on the reconstructed density field for this particular method (projected nearest neighbor and flux-limited tracer galaxies) as the majority of our studies of galaxy properties reported elsewhere are based on the density
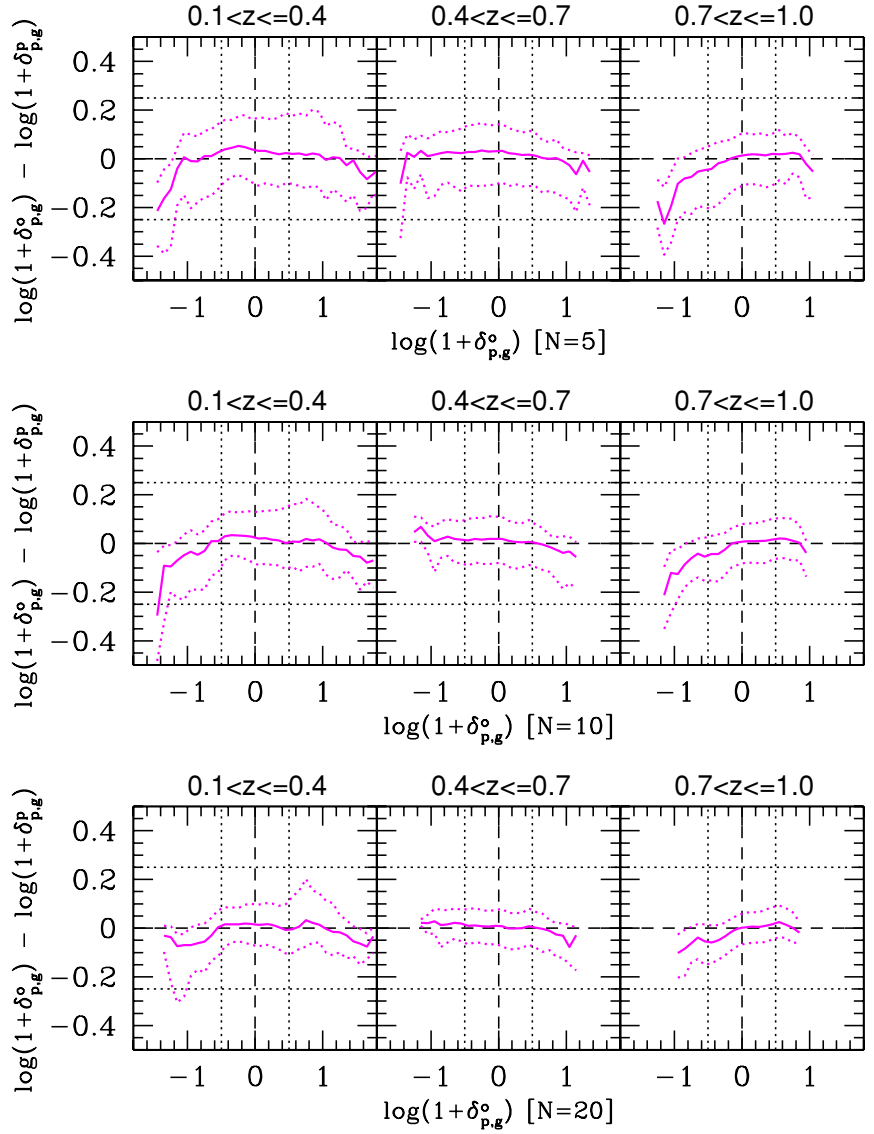

Figure 6. Difference between overdensities reconstructed in the observed catalog, using the ZADE formalism, and in the parent catalog, plotted as a function of the observed overdensity. The density is obtained by measuring distance to the fifth, 10th, and 20th nearest neighbor in the top, middle, and bottom panels, respectively. The continuous line represents median for the measured difference between the two reconstructed overdensities, the dotted lines correspond to the 25 th and 75 th percentiles for this difference. The indices are defined in Figure 4.

(A color version of this figure is available in the online journal.)

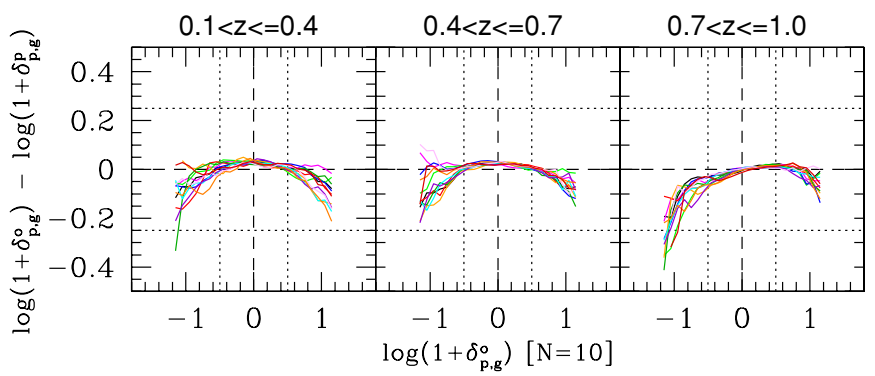

Figure 7. Difference between overdensities reconstructed in the observed catalog, using the ZADE formalism, and parent catalog, plotted as a function of the observed overdensity for the different mock catalogues. The density is obtained by measuring distance to the 10th nearest neighbor. Each continuous line corresponds to the median of the difference between the two reconstructed overdensities (observed and parent), in the bins of the observed overdensity, for a single mock catalog. Individual panels include results for 12 mock catalogs. The observed overdensities are presented in the range $-1.2<\log \left(1+\delta_{p, g}^{o}\right)<1.2$. The indices are defined in Figure 4.

(A color version of this figure is available in the online journal.)

field obtained by using Equation (4) and the apertures probed by this reconstruction method are smallest. We have carried out similar tests to obtain the uncertainties on the reconstructed overdensity when centered on a galaxy, using both fixed and adaptive 
apertures, following Equation (4), and flux- and volume-limited tracer galaxies, weighting them with $m_{i}=1, m_{i}=L_{B}$, and $m_{i}=M_{*}$. The statistical errors are comparable to the presented errors for the adaptive apertures defined by the same number of neighbors as discussed here and the fixed apertures starting from $3 h^{-1} \mathrm{Mpc}$.

\subsection{Comparison of the ZADE Approach to Simple Weighting} Schemes

The application of the ZADE approach in the density field reconstruction can be summarized as a novel treatment of objects in the input galaxy catalog for which a high-quality spectroscopic redshift is not available. This is based on an adjustment of the photo- $z$ likelihood distribution of these missing objects.

The traditional approach to the problem of how to deal with the "missing objects" (those with no spectroscopic redshift) would be to weight those objects with spectroscopic redshifts by the inverse of the spatial sampling rate, determined on some reasonably small angular scale. This is done in the DEEP2 (Cooper et al. 2007), VVDS (Cucciati et al. 2006; Marinoni et al. 2005), and locally in the SDSS (Kauffmann et al. 2004).

Here, we compare the overdensity fields reconstructed with the ZADE method and with the 10k sample galaxies weighted by the inverse of their sampling rate by simultaneously comparing them to the $40 \mathrm{k}$ overdensity field. The sampling rate $\omega_{i}(\alpha, \delta)$ at a given $(\alpha, \delta)$ position is defined by the fraction of the $10 \mathrm{k}$ galaxies in the 40k sample in some apertures. We define the sampling rate on a grid in R.A.-decl. plane with grid points separated by 0.5 arcmin, and the sampling rate by counting galaxies in the $10 \mathrm{k}$ and $40 \mathrm{k}$ samples in the squared R.A.-decl. apertures of 2 , 4 , and 6 arcmin. The results do not depend much on the box size used to measure the sampling rate, and in the following we use results obtained with the box size of 2 arcmin.

We reconstruct the mock overdensity field on the grid using the apertures defined by the $N$ th nearest neighbor, as defined in Section 5.1, but using only galaxies with the mock measured redshifts, i.e., the summation in Equation (1) goes only over galaxies in the 10k sample. For each galaxy $\phi_{i}=\omega_{i}(\alpha)$. To obtain overdensities, we use $\rho_{m}(z)$ obtained for the 40k mocks, as this allows us to explain any difference between the $1+\delta_{p}$ values in the $10 \mathrm{k}$ and $40 \mathrm{k}$ reconstructed overdensity field by a particular treatment of the objects without redshift. In reality, mean density obtained using only the $10 \mathrm{k}$ galaxies would be responsible for the additional uncertainty.

We demonstrate the difference between the 10k weighted observed and 40k parent overdensities using the same mock catalog as for the difference between the $10 \mathrm{k}+30 \mathrm{kZADE}$ and $40 \mathrm{k}$ reconstruction, inspecting the difference between the observed and parent overdensities as a function of the observed overdensities, expressed in $\log \left(1+\delta_{p}\right)$. The behavior of the median of this difference and the spread in this difference (quantified by the lower and upper quartiles in the distribution of these differences at a given $\log \left(1+\delta_{p}\right)$ value $)$ is similar for the rest of the mock catalogs.

First, we compare the overdensities reconstructed within the aperture defined by the fifth nearest neighbor in each of the catalogs (top panel in Figure 8). The offset between the $10 \mathrm{k}$ weighted and $40 \mathrm{k}$ overdensities is much larger than the offset between the $10 \mathrm{k}+30 \mathrm{kZADE}$ and $40 \mathrm{k}$ overdensities. In contrast to the $10 \mathrm{k}+30 \mathrm{kZADE}$ reconstruction, there is a trend to overestimate the value of the most overdense regions when using the weighted $10 \mathrm{k}$ sample. This systematic effect increases with redshift. When using the same number of objects to define
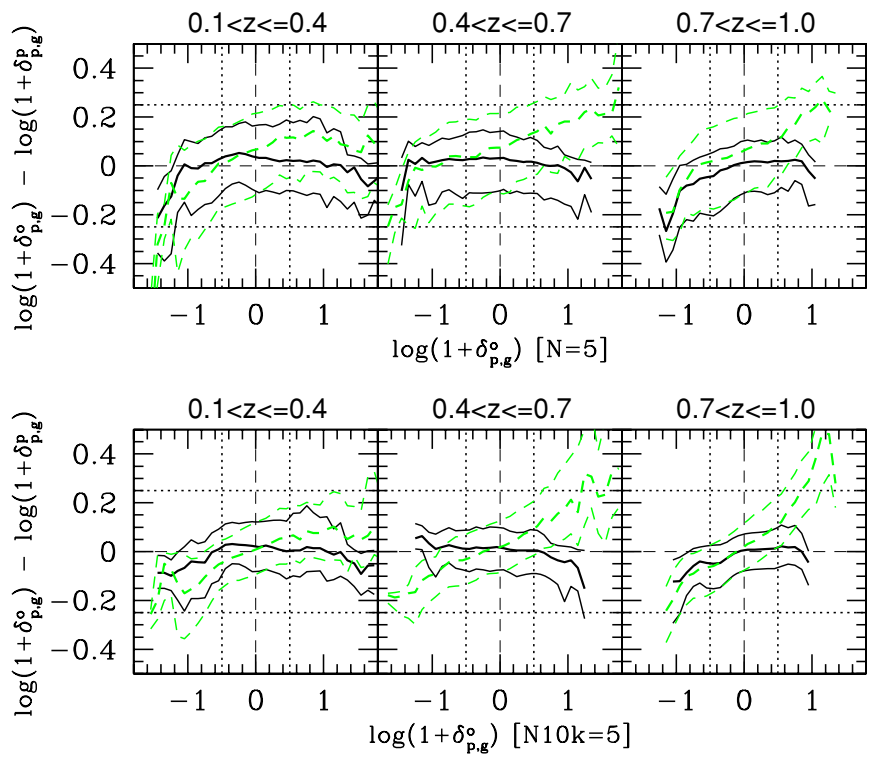

Figure 8. Comparison of the errors that exist between the 40k parent catalog and the reconstructions using the $10 \mathrm{k}+30 \mathrm{kZADE}$ approach (continuous curves) and a conventional weighting scheme applied to the $10 \mathrm{k}$ spectroscopic sample (dashed curved). In the top panels, the density is reconstructed in apertures defined by the distance to the fifth nearest neighbor in each of the catalogs. In the bottom panels, the density is reconstructed in apertures defined by the distance to the fifth nearest neighbor in the weighted 10k scheme, and to the 12 th nearest neighbor in the $10 \mathrm{k}+30 \mathrm{kZADE}$ and $40 \mathrm{k}$ scheme. The thick curves represent the median for the measured difference between the observed and parent reconstructed overdensities, the thin curves correspond to the 25 th and 75 th percentiles in the distribution of these differences. The indices are defined in Figure 4.

(A color version of this figure is available in the online journal.)

the aperture, therefore maintaining similar Poisson errors, the spread at a given reconstructed overdensity is slightly larger with the weighted $10 \mathrm{k}$ sample than with the $10 \mathrm{k}+30 \mathrm{kZADE}$ reconstruction. This will cause larger mixing of the overdense and underdense regions in the $10 \mathrm{k}$ weighted reconstruction than in the $10 \mathrm{k}+30 \mathrm{kZADE}$ reconstruction. However, because of the different number densities the fifth nearest neighbor samples the densities on different scales.

We therefore compare the overdensities reconstructed on the scale defined by the fifth nearest neighbor in the 10k catalog and 12 th nearest neighbor in the $10 \mathrm{k}+30 \mathrm{kZADE}$ and $40 \mathrm{k}$ catalogs, as the number density of objects is about 2.5 times higher in the $40 \mathrm{k}$ than in the $10 \mathrm{k}$ catalogs in the central $0.8 \times 0.8$ region (bottom panel of Figure 8 ). While the spread at a given reconstructed $\log \left(1+\delta_{p}\right)$ value is lower when using the comparable apertures in the weighted $10 \mathrm{k}$ and $40 \mathrm{k}$ samples, the systematic offset between the weighted $10 \mathrm{k}$ and $40 \mathrm{k}$ overdensities becomes larger, particularly in the overdense regions. The $10 \mathrm{k}+30 \mathrm{kZADE}$ reconstructed overdensity in the majority of regions reproduces the parent overdensity without the systematic offset.

It can also be seen that the range of the reliably reconstructed overdensity values is different. When using the same aperture, with the $10 \mathrm{k}+30 \mathrm{kZADE}$ we can reconstruct the parent overdensity values up to about $\log \left(1+\delta_{p}\right)=1 \pm 0.1$, while in the $10 \mathrm{k}$ weighted reconstruction we can reconstruct the parent overdensity values to about $\log \left(1+\delta_{p}\right)=0.6 \pm 0.1$ up to $z \sim 1$ (bottom panel of Figure 8). Likewise, the aperture (scale) at which we can reliably probe the density field is smaller when using the $10 \mathrm{k}+30 \mathrm{kZADE}$ reconstruction than when using the weighted $10 \mathrm{k}$ scheme, smearing small structures with the $10 \mathrm{k}$. This effect can be easily appreciated in the visual comparison of the 

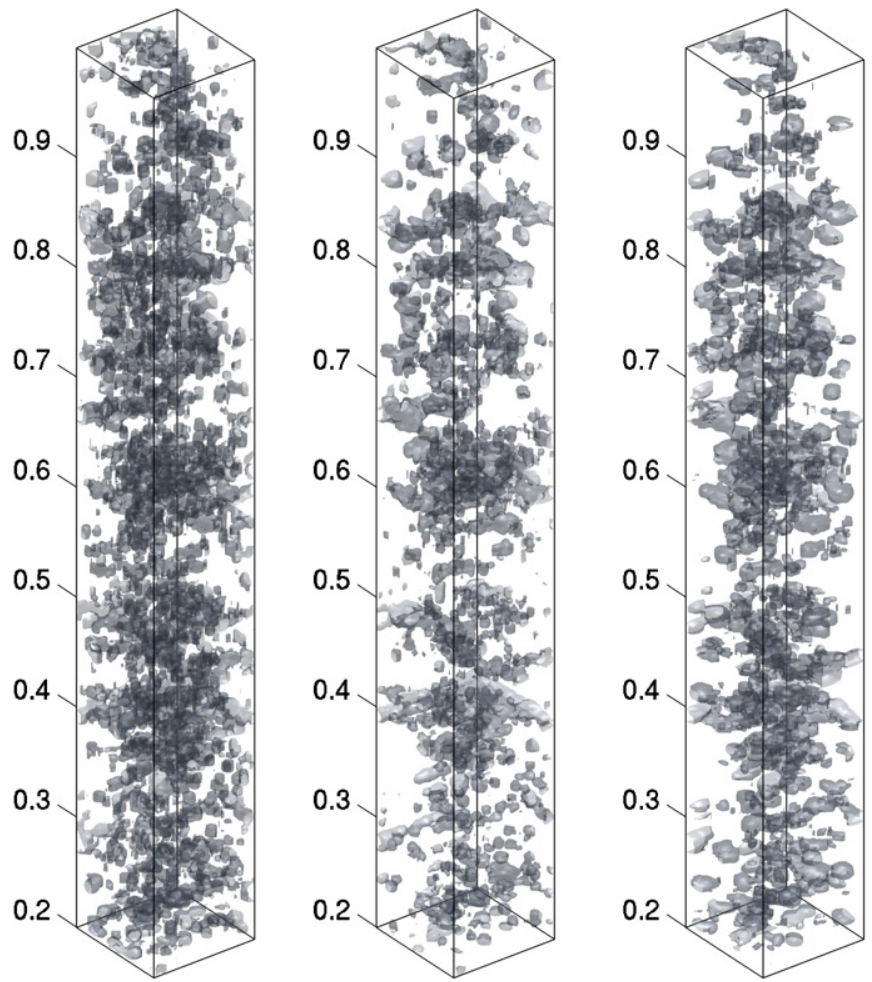

Figure 9. Mock overdensity field reconstructed using different reconstruction methods. The aperture is defined by the distance to the fifth nearest neighbor in the 40k (left), 10k+30kZADE (middle), and weighted 10k (right) catalogs. Only the structures enclosed within the regions $1+\delta_{p} \geqslant 3$ are plotted. Although both $10 \mathrm{k}$-related reconstruction do well, the ZADE approach captures better full dynamical range in the parent overdensities.

overdensity fields reconstructed with the 40k, 10k+30kZADE, and weighted 10k catalogs, presented in Figure 9. As we use the same $1+\delta_{p}$ value to define the isosurfaces in each of the overdensities, slight difference in the structures will be caused also by the offsets between the values of the parent and observed overdensities. Although both 10k-related reconstruction do well, the ZADE approach captures better the full dynamical range in the parent overdensities.

Obviously, depending on a science case, the various differences between the sampling-weighted and the ZADE reconstruction methods may be important.

\subsection{Additional Errors in the Galaxy Density Field Reconstruction}

We have discussed above the errors related specifically to the reconstruction method, i.e., the errors arising due to the fact that we use tracer galaxies with their measured properties instead of their true properties to reconstruct the density field. There are a number of additional uncertainties that arise, no matter which reconstruction method is used. Below, we address the uncertainties in the density field reconstruction that are present from the (finite) sampling of galaxies and due to working in the redshift space instead of real space, as well as due to the differences between the three-dimensional and two-dimensional, projected densities.

For this purpose, we concentrate on the overdensities reconstructed at the positions of the 10k galaxies, using Equation (4). We choose again the nearest neighbor apertures, as they are most commonly used in the scientific exploration of the zCOSMOS density field, even though they are more difficult to compare

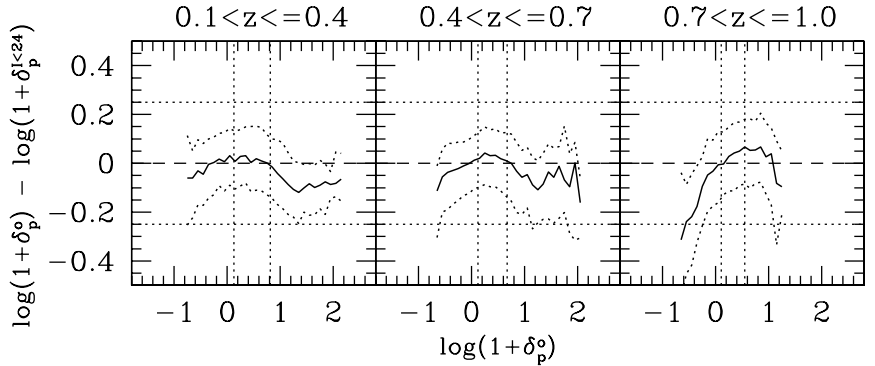

Figure 10. Difference between overdensities reconstructed in the observed catalog, using the ZADE formalism, and in the parent $100 \mathrm{k}$ catalog, plotted as a function of the observed overdensity. The density is obtained on a scale defined by the distance to the 10th and 25th nearest neighbors in the $10 \mathrm{k}+30 \mathrm{kZADE}$ and 100k sample of galaxies, respectively, as the mean density of objects in the $100 \mathrm{k}$ catalog is about 2.5 times higher than in the $40 \mathrm{k}$ catalog in the central $0.8 \times 0.8 \mathrm{deg}^{2}$ region (shown here). The continuous curve represents the median for the measured difference between the two reconstructed overdensities and the dotted curves correspond to the 25 th and 75 th percentiles for this difference. The horizontal dotted lines serve only as guidance lines, while the vertical dotted lines mark the values of the lower and upper quartiles in the observed overdensity distributions.

than the results with the fixed apertures. The results below are presented for the four randomly chosen mock catalogs (about 40,000 mock observed zCOSMOS galaxies).

\subsubsection{Sampling Density}

To address the issue how the sampling density affects the density field reconstruction, we compare the overdensity field at the positions of the $10 \mathrm{k}$ galaxies reconstructed using the $10 \mathrm{k}+30 \mathrm{kZADE}$ sample of $I_{\mathrm{AB}}<22.5$ galaxies to the overdensity field reconstructed using the sample of all $I_{\mathrm{AB}}<24$ galaxies, assuming all of these have reliably measured redshifts. Typically, in the mock catalogs there are $\sim 40,000$ galaxies down to $I_{\mathrm{AB}}<22.5$ and $\sim 110,000$ galaxies down to $I_{\mathrm{AB}}<24$ (100k sample from now on) in the area corresponding to that of zCOSMOS.

The resulting comparison of the $10 \mathrm{k}+30 \mathrm{kZADE}$ observed $\log \left(1+\delta_{p}^{o}\right)$ and the $100 \mathrm{k}$ parent $\log \left(1+\delta_{p}^{I<24}\right)$ overdensity field, reconstructed at the positions of the $10 \mathrm{k}$ galaxies, is presented in Figure 10. As the number of objects in the 100k sample is about 2.5 times higher than in the $10 \mathrm{k}+30 \mathrm{kZADE}$ sample, we compare the overdensity field when using the aperture defined by the 10th nearest neighbor for the $10 \mathrm{k}+30 \mathrm{kZADE}$ sample and by the 25 th nearest neighbor for the $100 \mathrm{k}$ sample.

The shape of the median curve of the distribution of differences between the $10 \mathrm{k}+30 \mathrm{kZADE}$ and $100 \mathrm{k}$ overdensities is similar to the shape of the median difference between the $10 \mathrm{k}+30 \mathrm{kZADE}$ and 40k overdensities (shown in Figure 6) at each reconstructed $10 \mathrm{k}+30 \mathrm{kZADE}$ overdensity. Overall, the median values are reconstructed rather well, with a tendency to underestimate the values of the 100k overdensity field in the regions of the lowest and highest observed underdensities when binning along the observed axis, as the 10k sample (and probably also the 40k sample) does not trace all of the possible structures defined by the $I_{\mathrm{AB}}<24$ galaxies.

The relevant question to address is whether this could affect our definition of the overdense and underdense regions. Both Cucciati et al. (2009) and Tasca et al. (2009) use percentiles in the distributions of the overdensity field to define the regions of various densities and to study galaxy properties, i.e., star formation indicators and morphology, respectively, as a function of galaxy environment. Similarly, we define here the most 
overdense regions as those with the overdensity higher than the value at the $75 \%$ of the overdensity distribution, the most underdense regions as those with the overdensity lower than the lowest $25 \%$ of the overdensity distribution and the regions of the mean overdensities all those between. Using the mocks and matching apertures (defined by the different number of neighbours in different samples), we estimate that $75 \%, 77 \%$, and $79 \%$ of the $10 \mathrm{k}$ galaxies which reside in the regions of the highest, mean, and lowest 100k overdensities, respectively, reside also in the regions of the highest, mean, and lowest $10 \mathrm{k}+30 \mathrm{kZADE}$ overdensities, respectively. The equivalent numbers when comparing the $40 \mathrm{k}$ and $100 \mathrm{k}$ overdensities measured at the positions of the $10 \mathrm{k}$ galaxies are $84 \%, 86 \%$, and $87 \%$. One can therefore expect that the "mixing" of the regions of the different $100 \mathrm{k}$ overdensities in the $10 \mathrm{k}+30 \mathrm{kZADE}$ sample is caused by similar contributions of the reconstruction errors $(10 \mathrm{k}+30 \mathrm{ZADE}$ to $40 \mathrm{k})$ and the errors caused by the differences in the distributions of the $10 \mathrm{k}$ and $100 \mathrm{k}$ galaxies.

\subsubsection{Relation to the "True" Overdensity Field}

As mentioned in Section 1, galaxies are biased tracers of the underlying matter distribution. It would be therefore interesting to compare the reconstructed overdensity field to the "true" matter overdensity field. However, reconstruction of the matter overdensity field is observationally a very challenging task (but see Section 6.3 for the dark matter reconstruction in the COSMOS field).

When using the density field reconstructed using the threedimensional distances in the redshift space and the top-hat smoothing filter (Equation (6)), a technique has been developed (Sigad et al. 2000) to infer the biasing relation between the galaxies and the matter. In a separate study, Kovač et al. (2009) find that zCOSMOS galaxies are nonlinear tracers of the underlying matter distribution in the both overdense and underdense regions. For the $M_{B}<(-20-z)$ sample, the linear bias is increasing from $1.24 \pm 0.11$ to $1.64 \pm 0.15$ over the redshift interval $0.4<z<1$.

To give an idea of how the "projected" type of the density field, commonly used to define the local galaxy environments because of peculiar velocities of galaxies, is biased relative to the true three-dimensional density, we compare the redshift space projected and real space three-dimensional overdensities. To avoid some additional uncertainties (i.e., sampling and the change of the number of tracers with redshift), we use for this comparison a more precise overdensity reconstructed from the catalog defined by a luminosity complete sample of galaxies with $100 \%$ sampling. We use the $M_{B}<-18$ limit (ignoring the redshift evolution for the moment), as this magnitude is the faintest for which the available $r \leqslant 26$ mock catalogs (Kitzbichler \& White 2007) are luminosity complete up to $z=1$. The typical number of galaxies in such a defined mock catalog of the zCOSMOS size is about 150,000 (150k and $150 \mathrm{kR}$ catalogs from now on for the redshift and real space, respectively).

Comparison of these two overdensity fields in $0.1<z<1$ is shown in Figure 11. The aperture is defined by the distance to the 20th nearest neigbor in both catalogs, as this is on average the same aperture as defined by the fifth nearest neighbor in the $10 \mathrm{k}+30 \mathrm{kZADE}$ sample. To correct for the projection effects, we scale the projected overdensities $\delta_{p}^{z}$ (in the redshift space) to the three-dimensional $\delta^{r}$ (in the real space) using $\left(1+\delta^{r}\right)=\left(1+\delta_{p}^{z}\right)^{(3 / 2)}$. The median projected overdensities correspond very well to the three-dimensional ones in the

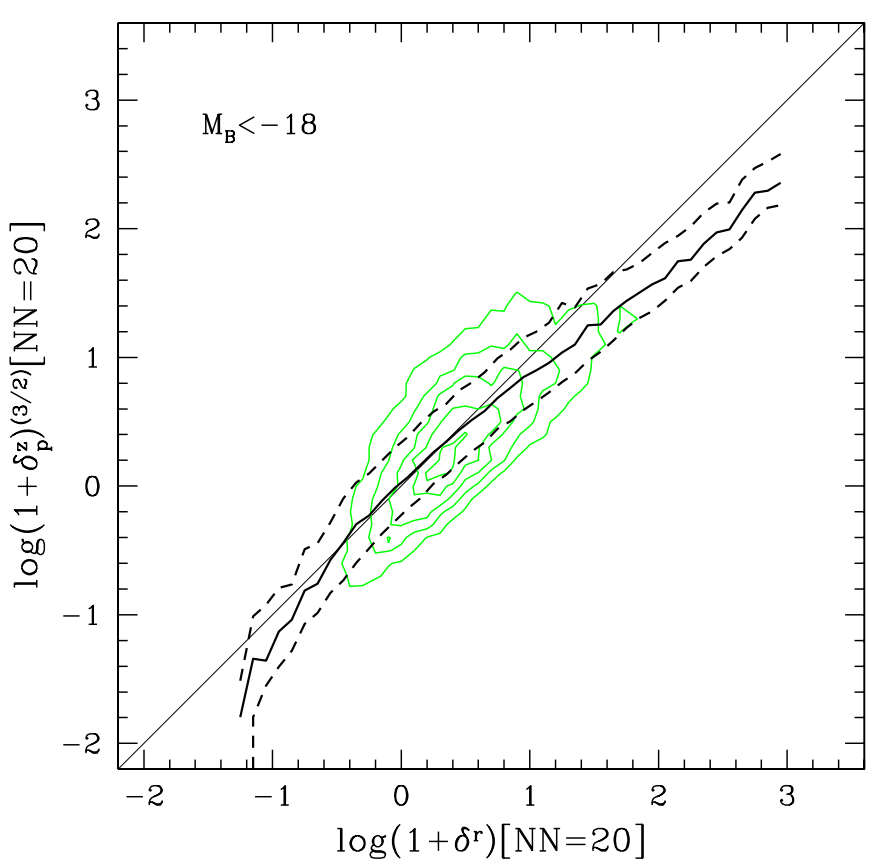

Figure 11. Comparison between the redshift space projected $\left(\delta_{p}^{z}\right)$ and the "true" real space three-dimensional $\left(\delta^{r}\right)$ overdensities in the $M_{B}<-18$ catalogs. To correct for the projection effects, we use $\left(1+\delta_{p}^{z}\right)^{(3 / 2)}$. The overdensities are reconstructed in $0.1<z \leqslant 1$ at the positions of the $10 \mathrm{k}$ mock galaxies using the apertures defined by the 20th nearest neighbor defined by the corresponding projected or three-dimensional distances. The contours represent the number density of galaxies with a given set of overdensities binned in $\Delta \log \left(1+\delta_{r}\right)=\Delta \log \left[\left(1+\delta_{p}^{z}\right)^{3 / 2}\right]=0.1$ intervals with a step of 50 . The thick continuous and dashed curves correspond to the median and upper and lower quartiles in the distribution of $\log \left[\left(1+\delta_{p}^{z}\right)^{3 / 2}\right]$ values at a given $\log \left(1+\delta_{r}\right)$.

(A color version of this figure is available in the online journal.)

regions of mean overdensities. The most overdense regions (in three-dimensional real space) are systematically underestimated in the projected density.

We have discussed in the previous section that the sampling of galaxies will cause the most overdense regions in the higher sampled catalogs to be somewhat underestimated by the $10 \mathrm{k}+30 \mathrm{kZADE}$ (see Figure 10). The same effect will exist also between the $10 \mathrm{k}+30 \mathrm{kZADE}$ and the $150 \mathrm{k}$ projected overdensities (using the apertures of comparable size). On top of this, the difference between the $150 \mathrm{k}$ projected and $150 \mathrm{kR}$ three-dimensional overdensities will cause even larger underestimation of the "true" $\left(1+\delta^{r}\right)$ values in the most overdense regions.

In summary, we would expect that all of the potential difficulties are in the direction of underestimating the densities in the most overdense regions. However, the effects are rather small in the regions occupied by most galaxies.

\subsection{Other Issues: Edge Effects}

For all the different types of the density estimators, the effect of the edges of the survey on the estimated density is to artificially lower the density, since galaxies are not detected outside of these edges. One of the ways to avoid this is to simply exclude all (grid) points at which the aperture size is larger than the distance to the nearest edge of the survey. However, in the case of a density estimated using the adaptive aperture, this approach will bias the density estimator by excluding regions of the lower density (larger adaptive aperture) in larger proportion than the denser regions (smaller adaptive aperture). 

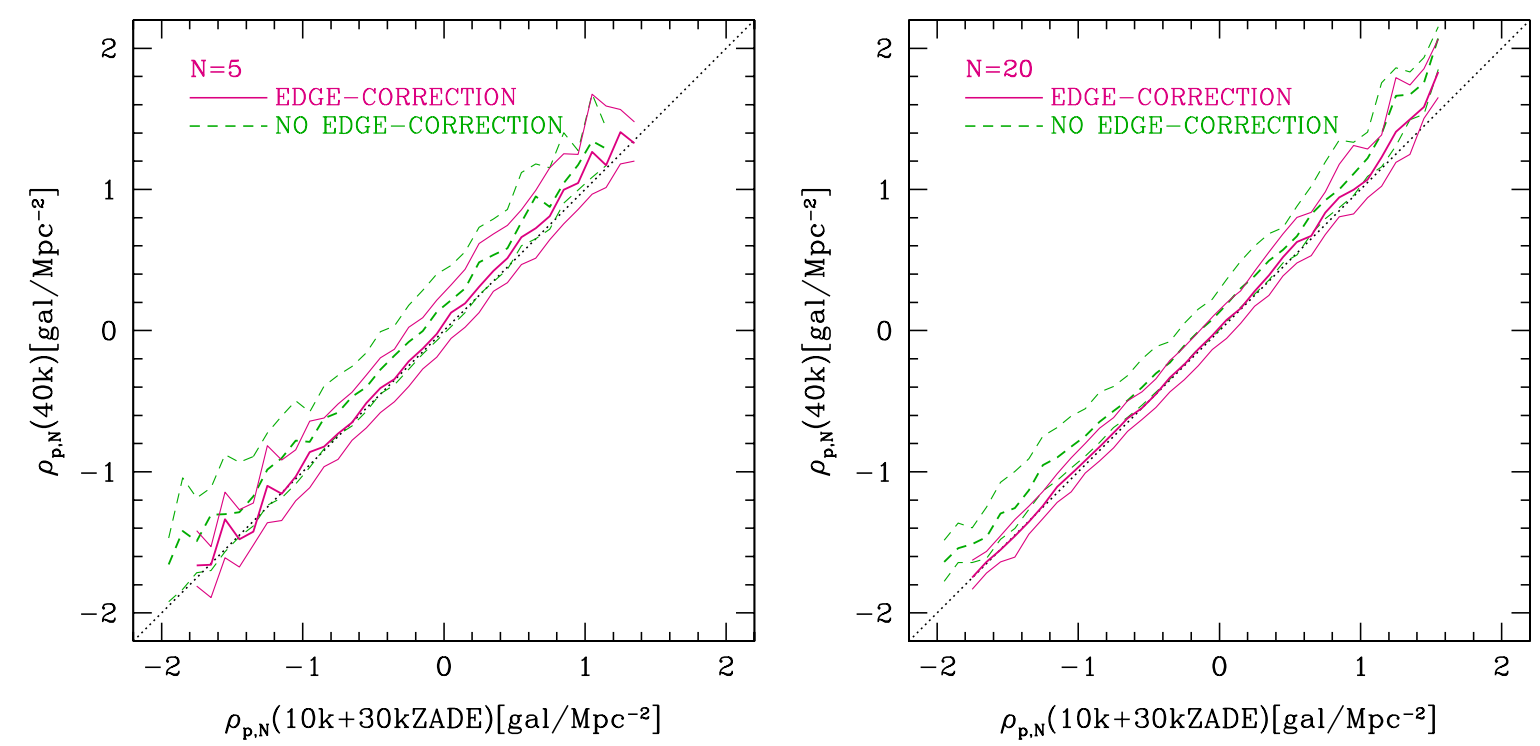

Figure 12. Effect of the edge-correction on the 10k+30kZADE densities. The medians (thick lines) and the lower and upper quartiles (thin lines) in the distribution of the 40k densities at a given value of the two different 10k+30kZADE densities are presented for the galaxies whose aperture partially extend outside of the survey edges. The results are presented with continuous lines when the edge correction has been applied, and with dashed lines in the case without the edge correction. The aperture for the density field reconstruction is defined by the distance to the fifth and 20th nearest neighbor in the left and right panels, respectively.

(A color version of this figure is available in the online journal.)

A common way to correct for the edge effects is to scale the measured density with the fraction of the adopted aperture which lies within the geometrical limits of the survey, assuming that the tracer galaxies in the part of the cell within and outside of the survey limits are distributed in the same manner.

For the adaptive density estimator the criteria to define the aperture close to the edges of the survey are nevertheless still affected by the edge. For example, in the case that the aperture is defined by the distance to the $N$ th nearest neighbor, only galaxies within the survey limits will be used to define this distance. Effectively, for the same density, the smoothing length will be larger for the points close to the edges than for the points within the survey, and this would artificially lower the densities near the edge due to the greater smoothing. Alternatively one could decrease the number of required neighbors close to the edges, according to the fraction of the volume within the survey limits. However, we find that correcting the densities at the points close to the survey limits by simply scaling the density by the fraction of the volume (area) within the survey limits already works reasonably well, and is certainly much easier to implement.

We demonstrate the effect of such an edge correction applied to the $10 \mathrm{k}+30 \mathrm{kZADE}$ reconstruction of the density field using four randomly chosen mock catalogs. As for the other tests, we first project galaxies within $\pm 1000 \mathrm{~km} \mathrm{~s}^{-1}$ of the point where the density is getting estimated, and then define the aperture by measuring the distance to the $N$ th nearest neighbor. We first reconstruct the density field at the positions of the zCOSMOS galaxies following the $10 \mathrm{k}+30 \mathrm{kZADE}$ scheme using only the inner $0.8 \times 0.8 \mathrm{deg}^{2}$ central region of the survey. For this purpose, the ZADE modified mock photometric redshifts for the $30 \mathrm{k}$ sample were recalculated using only the $10 \mathrm{k}$ galaxies within this $0.8 \times 0.8 \mathrm{deg}^{2}$ region. The final densities were estimated by dividing the reconstructed densities by the fraction of the circular aperture, with radius defined by the distance to the $N$ th nearest neighbor, within the $0.8 \times 0.8 \mathrm{deg}^{2}$ survey boundaries. We compare this to the density field reconstructed using the $40 \mathrm{k}$ galaxies in the full zCOSMOS area (about $1.3 \mathrm{deg}$ on a side).
In Figure 12, we compare the distribution statistics of the notcorrected and edge-corrected 10k+30kZADE densities to the $40 \mathrm{k}$ densities for the $10 \mathrm{k}$ galaxies from the inner $0.8 \times 0.8 \mathrm{deg}^{2}$ region for which the used aperture was partially outside of the surveyed region. The median values of the $40 \mathrm{k}$ densities match very well the edge-corrected $10 \mathrm{k}+30 \mathrm{kZADE}$ values, and they are systematically larger than the not-corrected $10 \mathrm{k}+30 \mathrm{kZADE}$ densities.

Encouraged by these tests, we therefore define for all of the density reconstructions the edge-corrected density estimate using the following equation:

$$
\rho_{c}=\rho / f \text {. }
$$

$\rho$ is estimated using Equation (1) and $f$ is the fraction of the adopted aperture that lies within the survey region. Depending on the scientific goals, one can exclude points with low values of $f$, if desired.

\section{THE 10K zCOSMOS OVERDENSITY FIELD}

\subsection{Reconstructed Environments of the zCOSMOS $10 \mathrm{k}$ Galaxies}

We present here the reconstructed environments of the $10 \mathrm{k}$ zCOSMOS galaxies obtained following the choices presented in Section 4.2. We discuss the obtained dynamical range, the dependence on the chosen aperture and on the weighting function $m_{i}$ of overdensity values quantified following Equation (4), where the overdensity is estimated centered on a galaxy. The apertures are defined by projecting galaxies within $\pm 1000 \mathrm{~km} \mathrm{~s}^{-1}$, and we omit direct reference to this projection in the following text.

We obtain the broadest dynamical range of reliably reconstructed local environments of the 10k zCOSMOS sample of galaxies by using the apertures defined by the distance to the fifth nearest neighbor in the flux-limited sample of tracer galaxies. Based on the tests on the mock catalog, 5 is the smallest number of neighbors which can be used to reliably reconstruct density at all redshifts probed. When using the larger number 


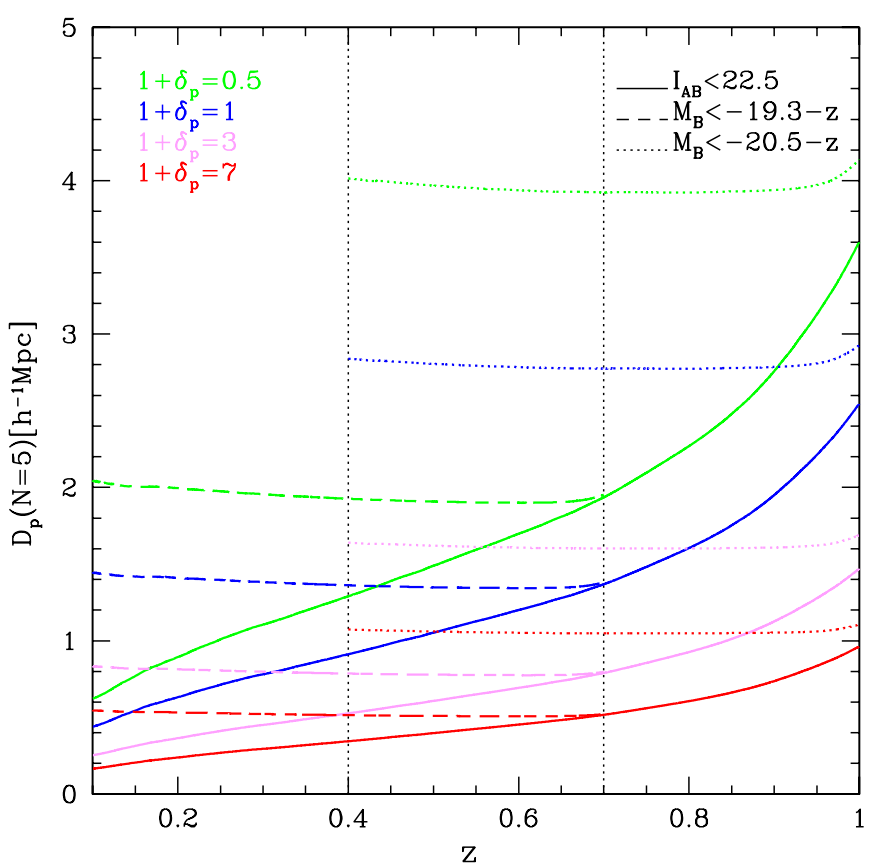

Figure 13. Projected distances, defined by the distance to the fifth nearest neighbor, used to define the aperture for the reconstruction of densities centered on the 10k zCOSMOS galaxies. The continuous, long-dashed, and short-dashed lines are for the $I_{\mathrm{AB}}<22.5, M_{B}<(-19.3-z)$ and $M_{B}<(-20.5-z)$ samples of tracer galaxies. The lines for the overdensities of $0.5,1,3$, and 7 in $\left(1+\delta_{p}\right)$ units are presented in green, blue, pink, and red, respectively.

of objects to define the aperture (e.g., 10 or 20), both the most overdense and underdense regions are smoothed out, particularly the most dense ones which have a smaller physical size. Therefore, the obtained overdensities with the volume-limited samples of tracer galaxies are also smoothed with respect to the overdensities reconstructed with the flux-limited sample of tracer galaxies.

The distances to the fifth nearest neighbor used to define the aperture for the reconstruction of the density centered at a
zCOSMOS galaxy for a set of $\log \left(1+\delta_{p}\right)$ values are presented in Figure 13, for all three samples of tracer galaxies. Rescaling this plot one can easily obtain the distances for the other values of $N$, since it will vary as $(N+1)^{(1 / 2)}$.

If we use fixed apertures, the smallest scale at which we are able to reliably reconstruct environments of the 10k zCOSMOS galaxies is about $3 h^{-1} \mathrm{Mpc}$ (based on the tests on the mock catalogs), using the density estimate given by Equation (4). On this and larger scales (see Figure 2), the dynamical range of the overdensities is smaller than when using the adaptive approach. The main point to be taken is that when using the fixed aperture to measure environments we are not able to differentiate between the most overdense regions that can be reconstructed at the adaptive scales.

The ranges of overdensities discussed above are obtained by weighting tracer galaxies with $m_{i}=1$. There is a good correlation between the number overdensities and the $L_{B}$ and $M_{*}$ weighted overdensities, because all are primarily set by the number of objects. However, the most overdense regions become even more overdense when using both $m_{i}=L_{B}$ and $m_{i}=M_{*}$, allowing even finer differentiation of the most overdense regions. When using the $M_{*}$ weighted counts of galaxies the dynamical range of overdensities is broader than when using the number or $L_{B}$ weighted galaxy counts. As an example, in Figure 14 we compare a subset of differently $m_{i^{-}}$ weighted reconstructed overdensities using the $M_{B}<(-19.3-$ $z$ ) tracer galaxies in $0.4<z \leqslant 0.7$, and aperture defined by the fifth nearest neighbor. Two effects are responsible for the observed scatter: the noise of the mapping between the number of galaxies and their $L_{B}$ luminosities or stellar masses $M_{*}$, and different luminosity (Zucca et al. 2009) or stellar mass (Bolzonella et al. 2009) functions in different environments.

Many of these effects can be seen in Figures 15 and 16, where we show the histogram distributions of overdensities reconstructed around the 10k zCOSMOS galaxies. The overdensities are reconstructed in the apertures defined by the 10th nearest neighbor (Figure 15) and by the fixed scale of $5 h^{-1} \mathrm{Mpc}$ (Figure 16), with three types of tracer galaxies: $I_{\mathrm{AB}}<22.5$
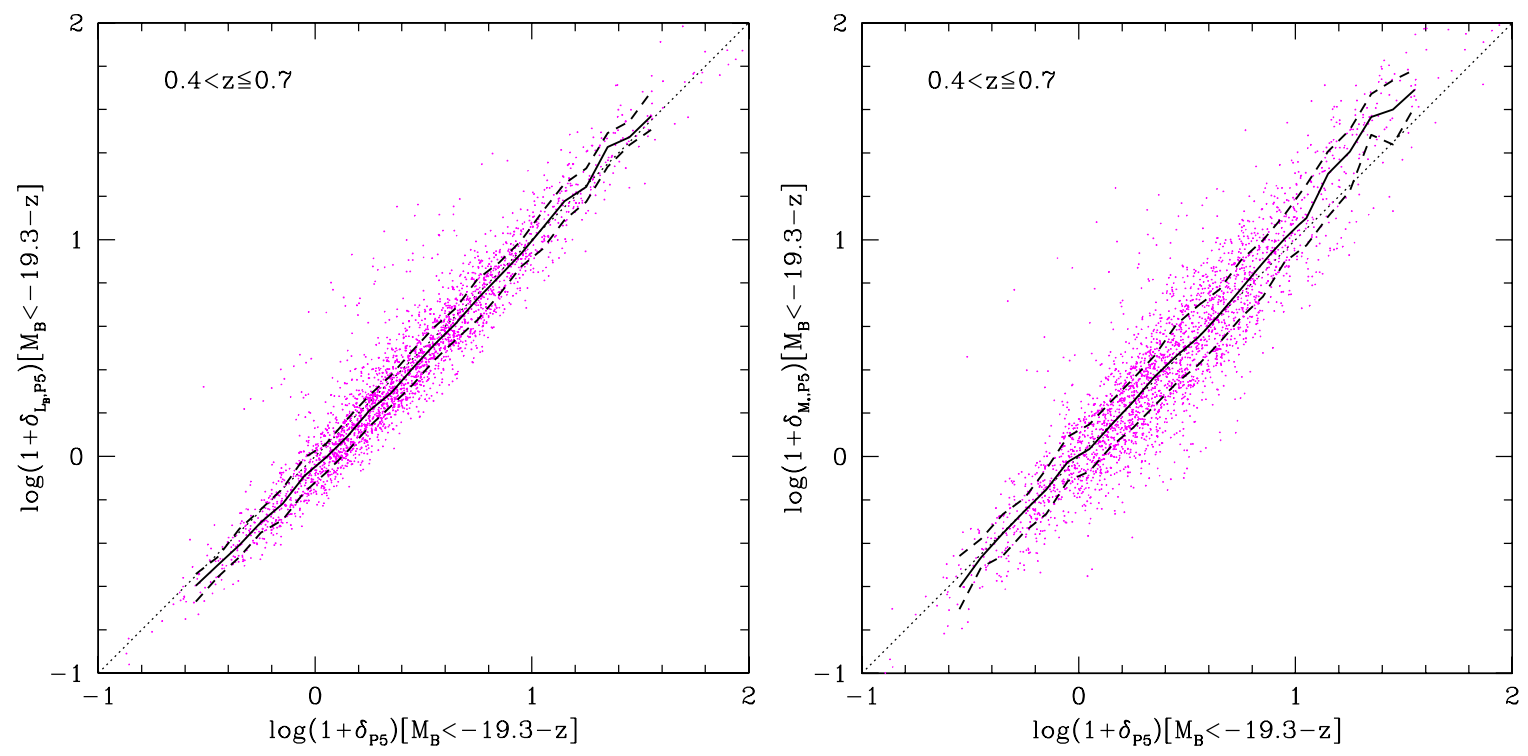

Figure 14. Comparison of the overdensities measured around the 10k zCOSMOS galaxies using Equation (4) with the $M_{B}<(-19.3-z)$ volume-limited sample of tracer galaxies and within the apertures defined by the distance to the fifth nearest neighbor. We compare the $B$-band luminosity and stellar mass weighted overdensities to the unity weighted overdensities in the left and right panels, respectively. The continuous thick curve is the median and the dashed thick curves are the lower and upper quartiles of the presented distributions, where the binning is carried out along the abscissa axis. There is a good correlation between the number overdensities and the $L_{B}$ and $M_{*}$ weighted overdensities. 

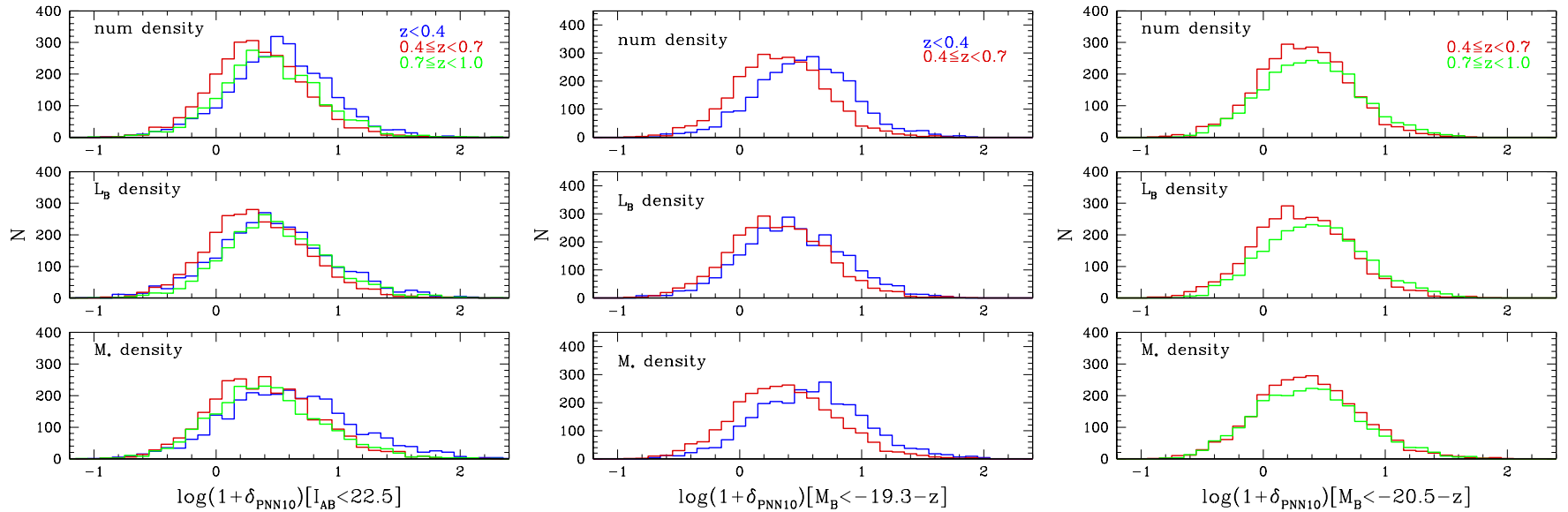

Figure 15. Histogram distributions of number of galaxies in the bins of overdensities $\log \left(1+\delta_{p}\right)$. The overdensities are reconstructed at positions of the $10 \mathrm{k} z \mathrm{COSMOS}$ galaxies, using the cylindrical adaptive filter $W$ (Equation (4)) with smoothing length defined by the 10th nearest neighbor. The distributions are shown separately for three different redshift bins: $z<0.4$ (blue), $0.4 \leqslant z<0.7$ (red), and $0.7 \leqslant z<1$ (green). We use mass-weighting $m_{i}=1, m_{i}=L_{B}$, and $m_{i}=M_{*}$ for the reconstructions presented in the upper, middle, and lower panels, respectively. A set of these three distributions of overdensities reconstructed for the $I_{\mathrm{AB}}<22.5$, $M_{B}<(-19.3-z)$ and $M_{B}<(-20.5-z)$ samples of tracer galaxies is presented in the left, middle, and right, respectively.
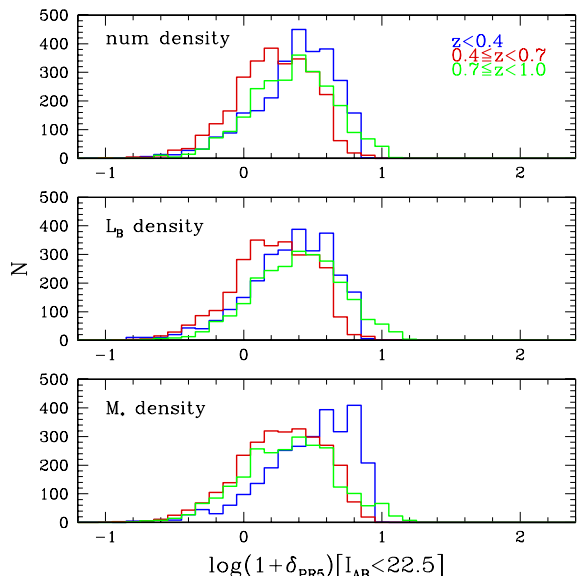
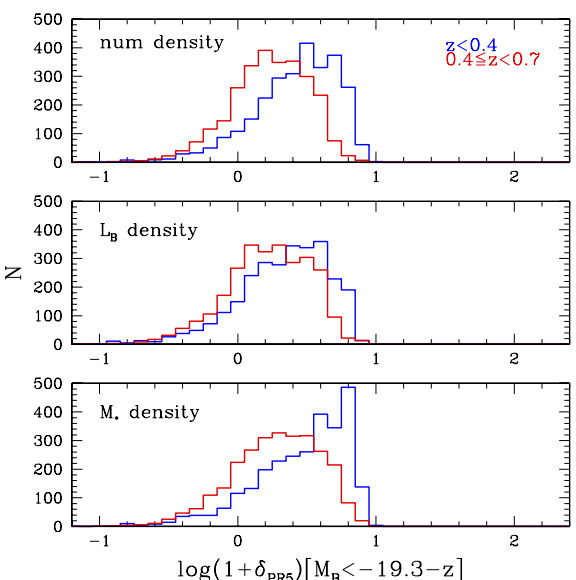
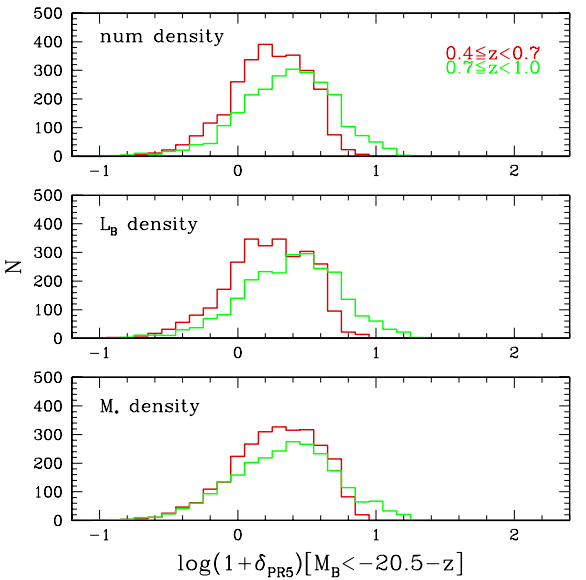

Figure 16. Histogram distributions of number of galaxies in the bins of overdensities $\log \left(1+\delta_{p}\right)$. The overdensities are reconstructed at positions of the $10 \mathrm{k} z \mathrm{COSMOS}$ galaxies, using the cylindrical fixed filter $W$ (Equation (4)) with smoothing length defined to be $5 \mathrm{~h}^{-1} \mathrm{Mpc}$. The distributions are shown separately for three different redshift bins: $z<0.4$ (blue), $0.4 \leqslant z<0.7$ (red), and $0.7 \leqslant z<1$ (green). We use mass-weighting $m_{i}=1, m_{i}=L_{B}$, and $m_{i}=M_{*}$ for the reconstructions presented in the upper, middle, and lower panels, respectively. A set of these three distributions of overdensities reconstructed for the $I_{\mathrm{AB}}<22.5, M_{B}<(-19.3-z)$, and $M_{B}<(-20.5-z)$ samples of tracer galaxies is presented in the left, middle, and right, respectively.

(left panels), $M_{B}<(-19.3-z)$ (middle panels), and $M_{B}<$ $(-20.5-z)$ (right panels) and three types of weighting: $m_{i}=1$ (top row panels), $m_{i}=L_{B}$ (middle row panels), and $m_{i}=M_{*}$ (bottom row panels).

The advantage of the use of the volume-limited sample is that, at every redshift, we are in principle using the same type of objects to define the environment, even though in practice the properties of these tracers can slowly change with redshift due to effects of evolution, which we cannot (yet) take out completely. The number density of these tracer galaxies is roughly constant, thus the Poisson noise and smoothing scale (if relevant) in the estimated density is on average the same at all redshifts.

On the other hand, when selecting galaxies in the fluxlimited sample, the number of available tracers is larger, and one can reliably reconstruct environments on smaller scales than when using the volume-limited sample. This can be critical for studying galaxy properties as a function of environment. However, different populations of galaxies will be used to define the environment at different redshifts and the typical smoothing scale will change with redshift in the adaptive approach. If we use the fixed scale, a different number of objects will be used systematically to measure the environment at different redshifts and therefore the noise component in the estimate will be different at different redshifts. Normalization to the mean overdensity (at a redshift of consideration) can overcome some of these effects.

\subsection{Cosmographical Tour of the 10k zCOSMOS Survey}

The global picture of the LSSs traced by the 10k zCOSMOS galaxies is obtained by the reconstruction of the density field on the grid filling the zCOSMOS volume. For the zCOSMOS data the overdensity is reconstructed on a regular grid with spacing of $1 h^{-1} \mathrm{Mpc}$ in all three directions (along the R.A., decl., and $z$ axis). All tracer galaxies within $\pm 1000 \mathrm{~km} \mathrm{~s}^{-1}$ are first set to the redshift of the grid point, and then used to obtain the projected distance to the fifth, 10th, and 20th nearest neighbor (as for the mocks). The presented overdensity field is the projected surface overdensity field $\delta_{p}$, and the values of the full three-dimensional overdensity field $\delta$ at the same smoothing scale $R$ would be larger, $1+\delta=\left(1+\delta_{p}\right)^{(3 / 2)}$. The overdensity field reconstructed 

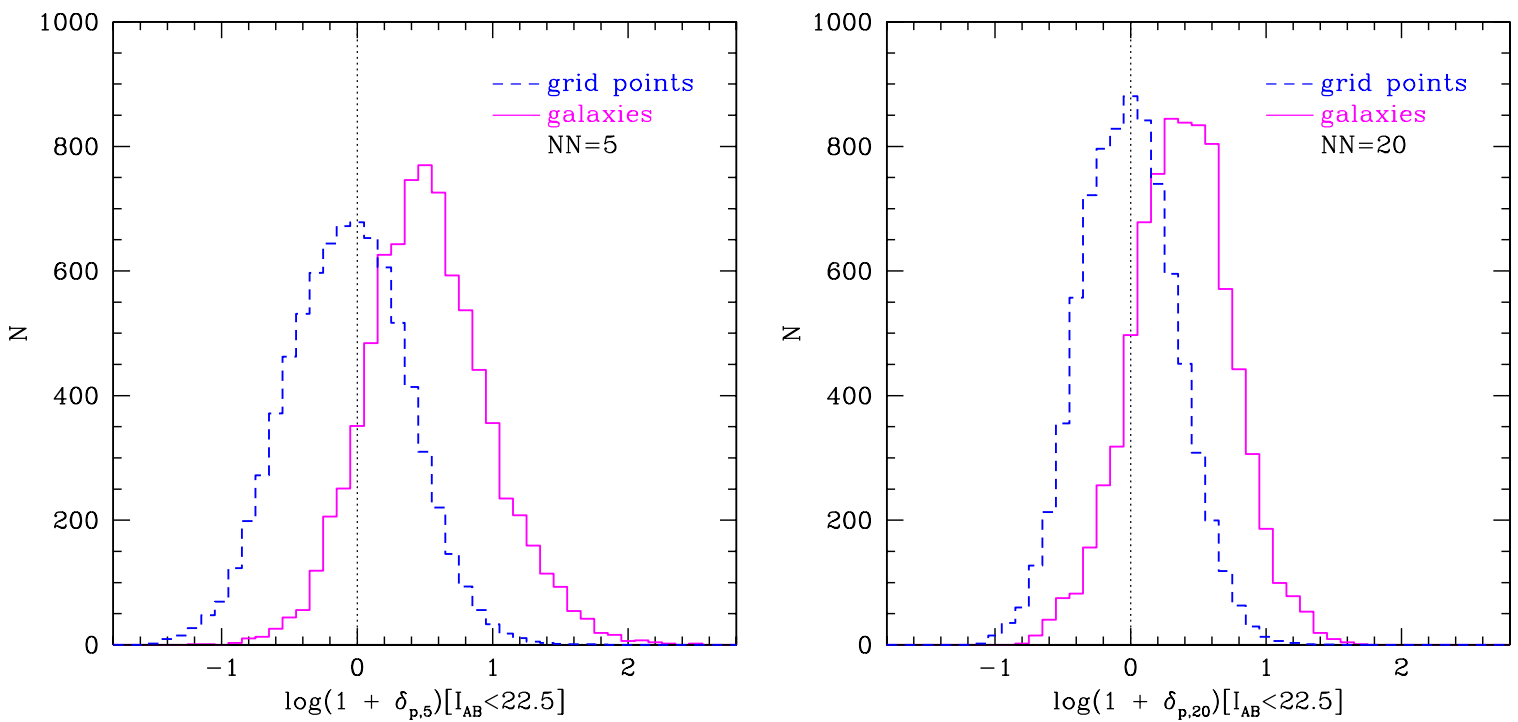

Figure 17. Histogram of the overdensities in the zCOSMOS volume calculated on the grid points (dashed line) and at the positions of zCOSMOS galaxies (continuous line). The histogram of the grid points is scaled to the number of galaxies with high-quality redshifts. Galaxies are not distributed homogeneously in the zCOSMOS volume, they form clumps of the overdense regions leaving some of the volume empty.

in this way is not suited for the cosmological analysis, it is more a compilation of local environments as they would be experienced by an arbitrary object residing in the zCOSMOS volume. We use this overdensity field for the cosmographical tour of the zCOSMOS survey.

We first compare the distributions of overdensities reconstructed with the flux-limited tracer galaxies on the grid, and at the positions of zCOSMOS galaxies, in Figure 17. The number of grid points is scaled to match the number of zCOSMOS galaxies with a high quality redshift in $0.1<z<1$. The smoothing of the overdensity structures is clearly visible in both the overdense and underdense tails when comparing the overdensity values reconstructed using the apertures defined by the 20th with respect to the fifth nearest neighbor, as might be expected. The most underdense regions seen on the grid are more underdense than the lowest $1+\delta_{p}$ values obtained centered on galaxies. The peak in distribution of overdensities at positions of galaxies is also shifted toward positive overdensity values (by $\log \left(1+\delta_{5}\right) \sim 0.5$ and $\log \left(1+\delta_{20}\right) \sim 0.4$ ) with respect to the overdensity field reconstructed on the grid points. Again, the distribution of overdensity values on the grid does not represent the universal volume distribution of overdense and underdense regions. In Kovač et al. (2009), we present the distribution of overdensity values obtained from the full three-dimensional density reconstruction on the fixed scale.

A visual representation of the $\mathrm{zCOSMOS}$ overdensity field is presented in Figures 18-20. For these presentations we use the overdensity field reconstructed on scales defined by the distance to the projected fifth nearest neighbor in the sample of fluxlimited tracer galaxies. Note that these imply that the actual smoothing scale is increasing with redshift (see Figure 13), and that different populations of galaxies are used in different redshifts to reconstruct the overdensity field. However, using the smoothing scale defined by the fifth nearest neighbor in the flux-limited sample we are able to obtain the broadest possible dynamical range of the overdensity field and to preserve at best the variety of the structures in the zCOSMOS survey. As Strauss \& Willick (1995) point out, a flux limited redshift survey “... is useful for qualitative and cosmographical description of the structures that are seen, and in some sense shows the maximum amount of information in the redshift survey."

The reconstructed overdensity field, presented in Figure 18, shows structures in a large range of comoving scales covering a spectrum of different overdensities at all redshifts reliably probed by the 10k zCOSMOS survey. Galaxies are distributed into cluster-like structures, surrounded by empty, void-like regions up to $z=1$. A few points should be noted. First, the increased smoothness and extension of the structures in the overdensity field toward higher redshift is an artifact of the increased smoothing scale with redshift. Second, for the smallest scale structures the imprint of the filter $W$ used is also clearly visible, especially in Figure 18. Even though sampling in the zCOSMOS field is not uniform, the resolution of similar structures is the same, given that we use ZADE to take into account galaxies without spectroscopic redshift and keep their position on the sky.

Figure 19 provides another view of the complex structure of this overdensity field, but compressed by a factor of $\sim 3.5$ in the redshift direction. Here, we present the LSS delineated by the isosurfaces enclosing regions with $1+\delta_{p} \geqslant 1.5,3,5$, and $10(1+\delta \geqslant 1.8,5.2,11.2$, and 31.6) going from the left to the right, respectively. The isosurface structures up to $1+\delta_{p} \geqslant 5$ are connected over the transverse comoving scales covering the full zCOSMOS area, and coherent over hundred or more comoving $h^{-1} \mathrm{Mpc}$ (or more than $\Delta z \sim 0.1$ ) in the radial direction.

Three large structures dominate the zCOSMOS cosmic web at $z \leqslant 1$. These structures are located at $z \sim 0.35$ (comoving distance $\left.D_{c} \sim 980 h^{-1} \mathrm{Mpc}\right), \sim 0.7\left(D_{c} \sim 1800 h^{-1} \mathrm{Mpc}\right)$, and $\sim 0.85\left(D_{c} \sim 2100 h^{-1} \mathrm{Mpc}\right)$ and they correspond to the peaks already visible in the number distribution of galaxies with redshift (see Figure 3). The connectivity of the two largest high redshift structures extend over $200 h^{-1} \mathrm{Mpc}$ in the radial direction (already visible at $1+\delta_{p} \geqslant 5$ ). Even though we are working with a flux-limited sample of tracer galaxies, meaning that we are detecting only the brightest and presumably most massive galaxies, at $z \sim 0.9$ the smoothing scale for the overdensities $1+\delta_{p} \geqslant 3$ are still below $1 h^{-1}$ Mpc (see 

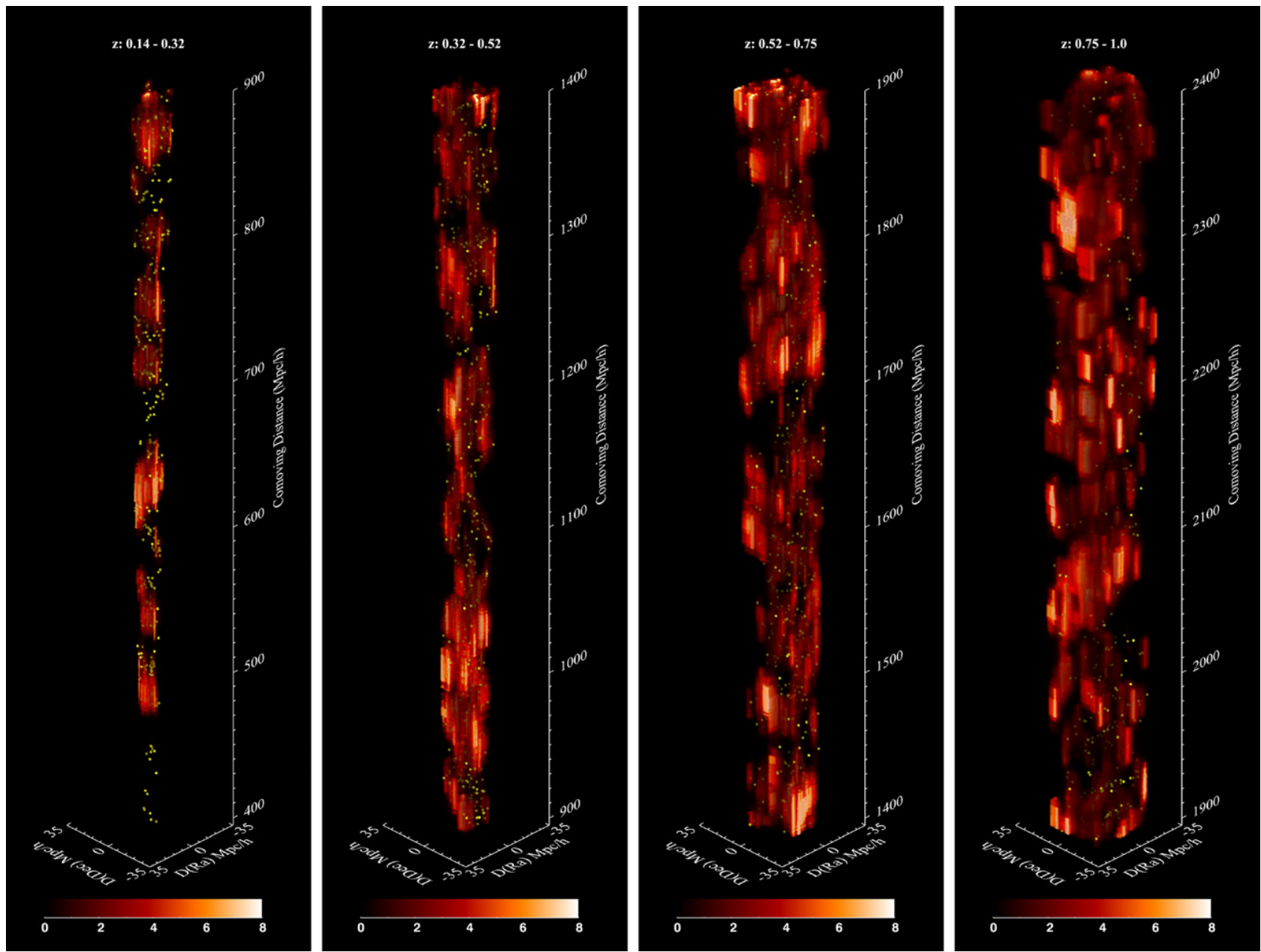

Figure 18. Cosmographical tour of the zCOSMOS survey. The 10k zCOSMOS overdensity field is reconstructed on the grid with the flux-limited $10 \mathrm{k}+30 \mathrm{kZADE}$ sample of tracer galaxies. The aperture is defined by the distance to the fifth nearest neighbor projected within $\pm 1000 \mathrm{~km} \mathrm{~s}^{-1}$ to redshift of the individual grid points. All three axis, distance from the survey center in right ascension, distance from the survey center in declination, and distance (corresponding to redshift), are expressed in comoving $h^{-1} \mathrm{Mpc}$. The reconstructed overdensity field yields structures in a large range of comoving scales covering a spectrum of different overdensities at all redshifts reliably probed by the 10k zCOSMOS survey. The color scale of the $1+\delta_{p}$ values is presented below each cone covering $500 h^{-1} \mathrm{Mpc}$ in redshift. The positions of galaxies are marked with dots. For clarity, only regions with $1+\delta_{p}<8$ are plotted.

(A color version of this figure is available in the online journal.)

Figure 13). Based on the tests on the mock catalogs, which include the selection effects of the 10k zCOSMOS survey, the existence of these huge overdense structures at high $z$ is not an artifact of our reconstruction method. The other striking element in the zCOSMOS overdensity field is a very small number of $1+\delta_{p} \geqslant 3$ (and above) structures in $0.4 \lesssim z \lesssim 0.6$ $\left(1100 h^{-1} \mathrm{Mpc} \lesssim D_{c} \lesssim 1580 h^{-1} \mathrm{Mpc}\right)$ except for an overdense sheet at $z \sim 0.53\left(D_{c} \sim 1420 h^{-1} \mathrm{Mpc}\right)$.

Complementary to the large positively overdense structures, we identify in the zCOSMOS $1+\delta_{p}$ field also the structures enclosed by the overdensity values of $1+\delta_{p}=0.15(1+\delta=0.06)$ and $\delta_{p}=0.25(1+\delta=0.13)$ which contain only regions with $1+\delta_{p}$ lower than these (the underdense regions, Figure 20). The identified regions are at least 6.67 and 4 times less dense than the mean density, respectively. These regions with low galactic density do not show strong clustering in either the transversal (R.A.-decl.) or radial (redshift) direction. Also, they appear to be more homogeneously distributed over the zCOSMOS redshift range, even though they are less present in the redshift ranges of the three most overdense structures, being particularly absent at $z \sim 0.35\left(D_{c} \sim 980 h^{-1} \mathrm{Mpc}\right)$.
Clearly, the amount of LSS in the zCOSMOS field varies with redshift. Quantitatively, this cosmic variance of the observed structures is shown in the left panel of Figure 21, where we plot the fraction of the volume of the zCOSMOS survey enclosed within the isosurface structures of a given $1+\delta_{p}$ value. We split the sample into four $\Delta z=0.2$ slices, starting from $z=0.2$. The fraction of the volume occupied by the structures with at least $1+\delta_{p}$ values is rapidly decreasing with $1+\delta_{p}$. The two higher redshift slices are statistically more representative (they occupy larger volumes), and we see that for the slices above $z=0.6$ about $44 \%$ of the volume is in the structures with $1+\delta_{p} \geqslant 1$, and about $10 \%$ of the survey volume is in the structures with $1+\delta_{p} \geqslant 3$. The cosmic variance is particularly noticeable between the two lower redshift slices. At $1+\delta_{p}=3$ and $1+\delta_{p}=15$, the volume fractions in those structures in the two lower redshift slices are different by a factor of $\sim 3$ and $\sim 4$, respectively.

As expected, the fraction of galaxies residing in the isosurface overdensity structures is much higher than the volume fraction of these structures. Taking the two higher redshift slices as statistically more representative, we conclude that about $50 \%$ 

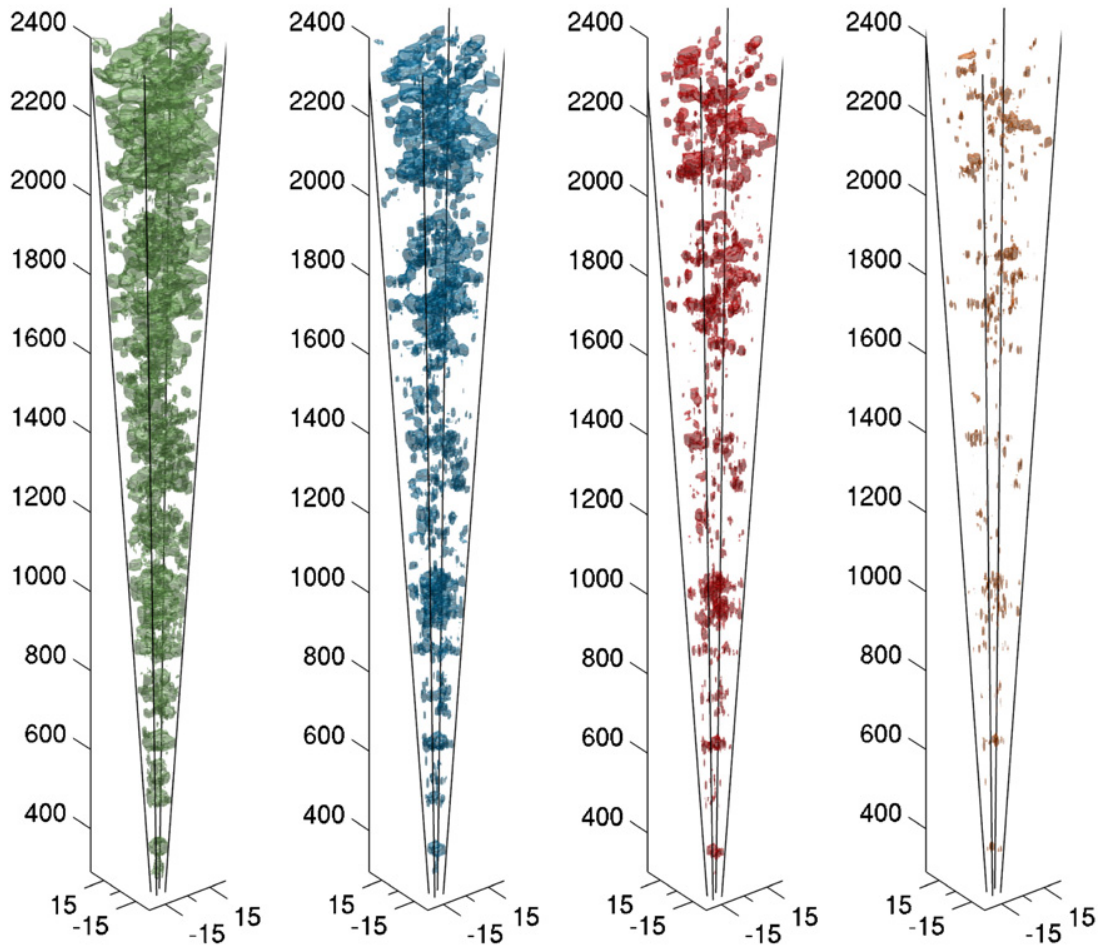

Figure 19. Distribution of the overdense structures in the $10 \mathrm{k}$ zCOSMOS overdensity field. The structures are defined as isosurfaces enclosing regions with $1+\delta_{p} \geqslant$ $1.5,3,5$, and $10(1+\delta \geqslant 1.8,5.2,11.2$, and 31.6) from the left to the right, respectively. Comoving distances in the radial direction correspond to $0.1<z<1$. The distribution of overdense structures matches the richness and complexity of the local cosmic web up to $z \sim 1$. The structures are delineated from the overdensity field reconstructed following the same scheme as in Figure 18. All three axis, distance from the survey center in right ascension (left-hand side axis), distance from the survey center in declination (right-hand side axis), and distance (corresponding to redshift; vertical axis) are expressed in comoving $h^{-1} \mathrm{Mpc}$. The figures are compressed by a factor of $\sim 3.5$ in the redshift direction.

(A color version of this figure is available in the online journal.)

of the galaxies reside in structures with $1+\delta_{p} \geqslant 3.5$, while about $50 \%$ of the stellar mass and about $50 \%$ of the $B$-band luminosity at a given $z$ inhabit the structures with $1+\delta_{p} \geqslant 4$ (three right panels in Figure 21). This reflects the known fact that galaxies preferentially live in the overdense regions (also seen in Figure 17). The main point of these plots is to give an idea what is the fraction of galaxies and their stellar mass and luminosity enclosed within the isosurface structures presented in Figures 19 and 20 with respect to all galaxies in the zCOSMOS volume.

\subsection{Comparison to the Other LSS Measures}

The high resolution of the zCOSMOS-bright spectra allows also to identify virialized groups of galaxies with velocity dispersion of $\sim 250 \mathrm{~km} \mathrm{~s}^{-1}$. Knobel et al. (2009) have applied the friends of friends and the Voronoi based group finding algorithms to the zCOSMOS galaxies with the high confidence redshift. In total, their optimal group catalog contains 800 groups with at least two detected members up to redshift of one in the zCOSMOS volume. From these, 102 groups have at least five members detected. Tests on mocks show that the vast majority of all of the identified groups will be virialized objects.

The other tracer of the virialized structures is the hot baryonic gas detected in the X-rays. Finoguenov et al. (2007) carried out the identification of the X-ray clusters in the COSMOS field using the XMM-Newton observations. The updated cata$\log$, improved with new additional X-ray data and using the spectroscopic zCOSMOS redshifts, contains $218 \mathrm{X}$-ray clusters detected with high confidence (A. Finoguenov et al. 2010, in preparation).
A comparison of the overdensities reconstructed at the positions of all zCOSMOS galaxies and of those galaxies residing in the virialized structures, whose detection is at least partially based on the zCOSMOS redshifts, is presented in Figure 22. The majority of galaxies detected in the X-ray clusters or the richer optical groups reside in extremely overdense regions. The galaxies defining the poorer groups extend to much lower overdensities than the rich $N \geqslant 4$ optical groups and X-ray clusters, and they almost completely avoid the most overdense regions.

The previous studies of the LSSs in the COSMOS field were based exclusively on the use of the photometric redshifts. Guzzo et al. (2007) used projected photometric redshift densities, $\mathrm{X}$-ray surface brightness and one of the first weak-lensing convergence maps to describe the extended structure at $z \sim 0.7$ in the COSMOS field. Scoville et al. (2007b) carried out the identification of LSSs by adaptive smoothing of galaxy counts in the COSMOS field up to $z<1.1$ using the photometric redshift catalog. Based on a sample of about $1.5 \times 10^{5}$ galaxies down to $I_{\mathrm{AB}}<25$ with redshift uncertainty $\sigma_{z} \leqslant 0.1$ (but which have improved in the mean time, see Ilbert et al. 2009; Salvato et al. 2009), Scoville et al. (2007b) found 42 LSSs and clusters.

Moreover, based on the shear maps in the COSMOS field, Massey et al. (2007) reconstructed the distribution of the dark matter projected along the line of sight up to $z \sim 1$, using the shape information for about half a million galaxies. The threedimensional distribution of dark matter has been obtained from the differential growth of the lensing signal between many thin slices separated by $\Delta z=0.05$, using the photometric redshift information. While the detailed comparison of the dark matter mass and the zCOSMOS galaxies overdensity distribution is out 

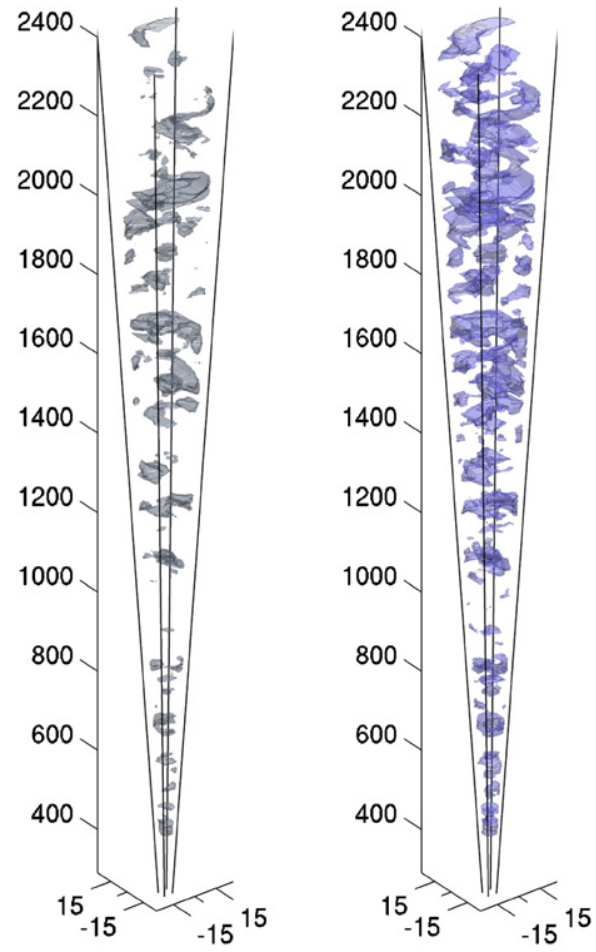

Figure 20. 10k zCOSMOS underdense regions (voids). The structures are defined as isosurfaces enclosing regions with $1+\delta_{p} \leqslant 0.15$ (6.67 times smaller than $\rho_{m}$ ) and 0.25 (4 times smaller than $\rho_{m}$ ) in the left and the right cone, respectively. Comoving distances in the radial direction correspond to $0.1<z<1$. The structures are delineated from the overdensity field reconstructed following the same scheme as in Figure 18. All three axes, distance from the survey center in right ascension (left-hand side axis), distance from the survey center in declination (right-hand side axis), and distance (corresponding to redshift; vertical axis), are expressed in comoving $h^{-1} \mathrm{Mpc}$. The figures are compressed by a factor of $\sim 3.5$ in the redshift direction.

of the scope of this paper, based on the visual inspection (see Figure 5 in Massey et al. 2007 and our Figure 19) it is clear that the resolution in the radial dimension in the reconstructed structures is much higher when using spectroscopic redshifts of galaxies than the weak lensing signal. Moreover, it is the synergy of the both galaxy LSSs and shear maps which will likely produce the distribution of dark matter mass of higher radial resolution.

In Figure 23, we present the visual comparison of the zCOSMOS overdensity field with the bound structures: LSSs identified from only the photometric redshifts (left; Scoville et al. 2007b), X-ray clusters (middle panel; A. Finoguenov et al. 2010, in preparation), and zCOSMOS groups with at least three members (right panel; Knobel et al. 2009). There is an overall good correspondence of the overdense regions and the bound structures in the zCOSMOS volume, particularly those identified using the spectroscopic redshifts. Almost no virialized structure is detected in regions with $1+\delta_{p}<3$. The agreement with the structures from the photometric redshifts is particularly good in the regions of the structures which extends over large redshift intervals. Also, one needs to keep in mind that the exact redshift position of these structures is more uncertain.

More detailed insight into the spatial distribution of the overdense and virialized structures identified by using the zCOSMOS spectroscopic redshifts at least in part (Knobel et al. 2009; A. Finoguenov et al. 2010, in preparation) is presented in the panels of Figure 24. We use the same data as in the previous figure, but now the overdensity field is projected in redshift slices of $\Delta z=0.025$ width, starting from redshift 0.2 . We use the cut of $1+\delta_{p} \geqslant 6.67$ to define the overdense regions, higher than in Figure 23. For a contrast, we also plot the equivalently underdense regions defined by isosurfaces with $1+\delta_{p} \leqslant 0.15$. The X-ray structures and the optically defined groups with at least three detected members reside inside of the LSSs defined by the chosen overdensity value in most of the cases in the whole redshift range $0.2<z<1$. However, there are some virialized structures which do not live in these most overdense regions. In fact, as we have already seen in Figures 22 and 23, the virialized structures trace also the less overdense regions. Moreover, the apparent overlap between some of the virialized structures and underdense regions in Figure 24 is only due to the projection effects.

\subsection{Comparison of the $10 \mathrm{k}$ zCOSMOS LSS to the LSS in the Mock catalogs}

The overdensity field reconstructed using the galaxies detected in the zCOSMOS survey is highly complex, resembling the network of the local cosmic web up to the highest redshift $z=1$ probed. We compare here the zCOSMOS overdensity field to the overdensity fields in the "10k+30kZADE" mock catalogs. The mock catalogs and the density field reconstruction procedure on the mocks were discussed in Section 5.1. For the exact comparison of the data and the mock overdensity field, we reconstruct the overdensity field of the 10k zCOSMOS galaxies on the same grid as was used for the mock catalogs, defined by $\Delta \alpha=\Delta \delta=2 \operatorname{arcmin}$ and $\Delta z=0.002$ and using the flux-limited tracers of galaxies. We did not calculate the edge corrections for all the mock catalogs (which is a very time consuming process computationally), and instead compare the overdensity fields
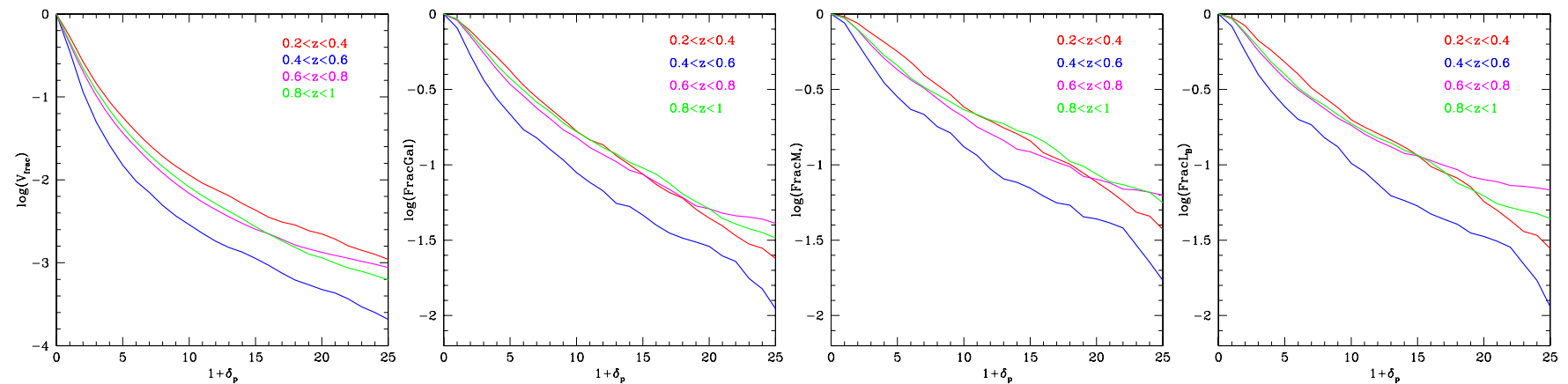

Figure 21. Fractions of volume, galaxies, stellar masses, and $B$-band luminosities enclosed in the structures defined by the given overdensity $1+\delta_{p}$ values, going from the left to the right. These fractions are presented in narrow redshift slices $\Delta z=0.2$, starting from $z=0.2$. The resulting fraction are dominated by cosmic variance. Note the different scale of the $y$-axis in the first panel. 

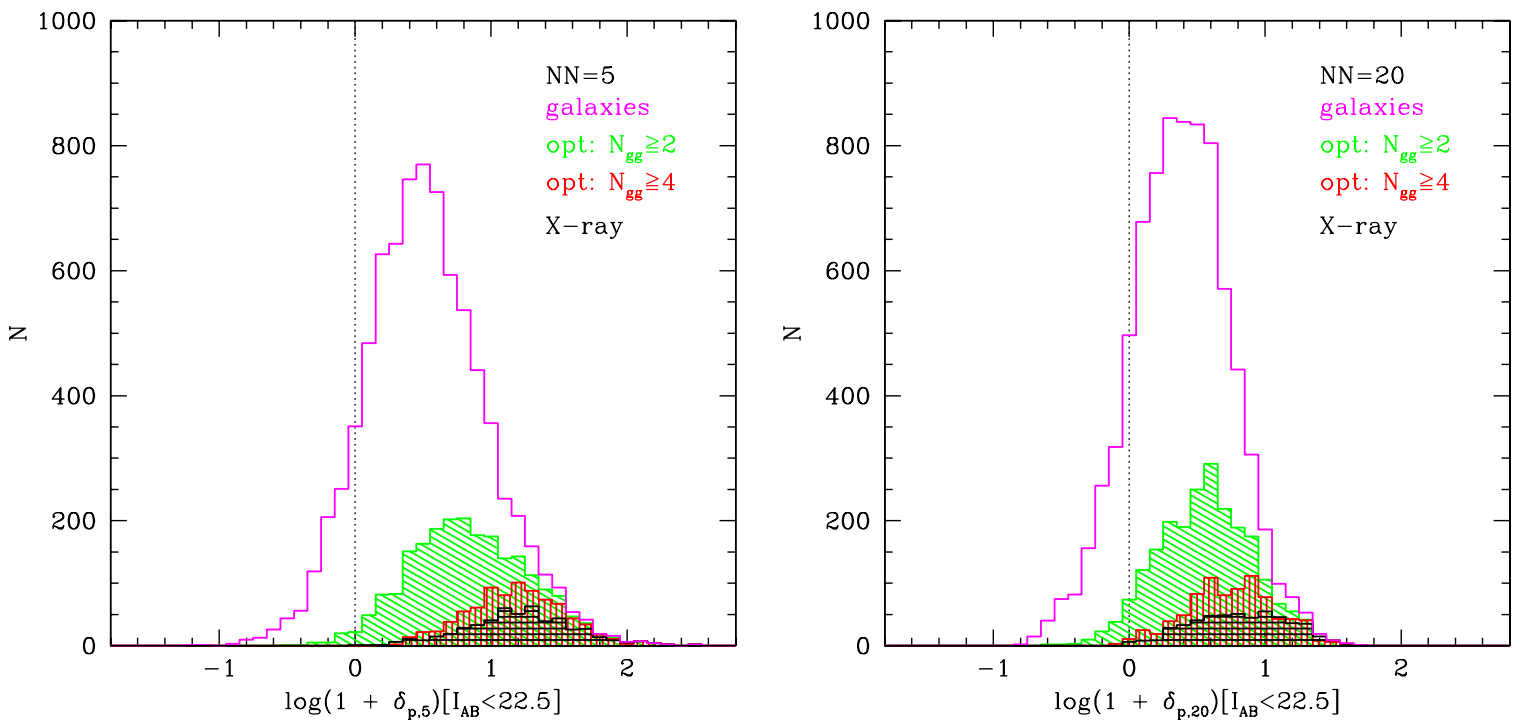

Figure 22. Comparison of the overdensities reconstructed at the positions of all zCOSMOS galaxies and those galaxies residing in the virialized structures identified using at least partially zCOSMOS redshifts. The empty histogram is obtained for the overdensities at the positions of zCOSMOS galaxies. The shaded histograms represent the distribution of overdensities centered on galaxies which reside in the virialized structures: X-ray clusters (horizontal lines), the optical groups with at least two detected members (30 deg inclined lines), and the optical groups with at least four detected members (vertical lines). The histograms overlap each other.

(A color version of this figure is available in the online journal.)

uncorrected for edge effects for both the data and the mocks. Given that we are only interested in comparing the structures in the overdensity fields in the data with structures in the mocks, and not to draw any scientific conclusions from the distributions of the detected structures, this should not matter.

In Figure 25, we show the three-dimensional distribution of the $1+\delta_{p}=3$ isosurfaces in the overdensity field estimated on the angular grid of the 10k zCOSMOS galaxies and of the mock catalogs. The visual inspection of the LSS defined by the $1+\delta_{p}=3$ isosurfaces leaves the impression that there are more large contiguous structures in the real data than in the mock catalogs.

For a quantitative comparison of the overdensity field of the real zCOSMOS and mock catalogs we have implemented a volume filling statistic. We calculate the fraction of the survey volume in which the overdensity value is above a given threshold. As a reference, we use the value obtained by averaging the individual statistics from the 12 mock catalogs. We use the standard deviation of the 12 mock results as an error estimate, which is dominated by the cosmic variance.

First, we compare the overall distribution of the survey volumes in structures above a given $1+\delta_{p}$ value in the $0.2<z<$ 1 redshift range using the overdensity field reconstructed in the apertures defined by the distance to the 10th nearest neighbor of the flux-limited tracer galaxies (Figure 26, left). Even though we use the angular grid to reconstruct the density field, we count the comoving volumes (in $\left.\left(h^{-1} \mathrm{Mpc}\right)^{3}\right)$ of the individual cells to properly calculate these volume fractions. There is an excellent agreement, within $1 \sigma$ errors, between the fractions of the volumes in the isosurface structures in the data and in the average mock.

We carry out the same analysis dividing the redshift range in four intervals: $0.2<z<0.4,0.4<z<0.6,0.6<z<0.8$, and $0.8<z<1$ (Figure 26, right). While the overdensity distributions of the data in $0.2<z<0.4$ and $0.6<z<0.8$ redshift ranges are in relatively good agreement with the mock results, the redshift interval $0.4<z<0.6$ is underdense in the data with respect to the mocks, while the situation is reversed
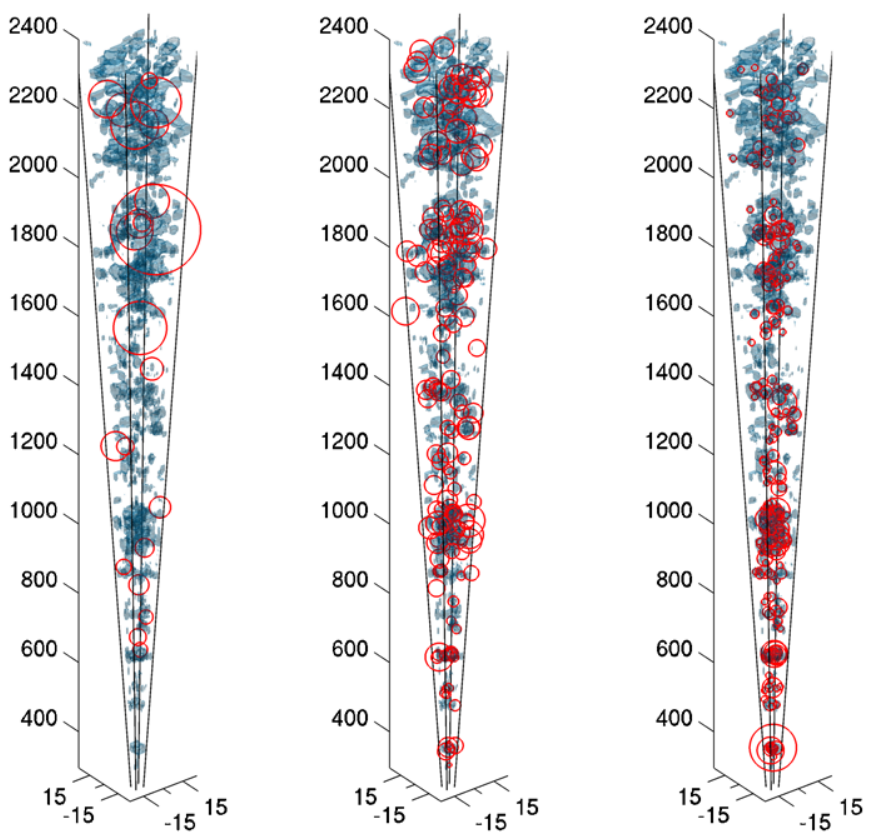

Figure 23. Comparison of the zCOSMOS density field to the estimates of the bound structures. The underlying zCOSMOS density field at $1+\delta_{p} \geqslant 3$ is obtained with the 10k+30kZADE flux-limited sample and the apertures defined by the distance to the 10th nearest neighbor projected within $\pm 1000 \mathrm{~km} \mathrm{~s}^{-1}$. On top of the overdensity field are marked with circles overlaid LSSs identified using the photometric redshifts only (left; Scoville et al. 2007b), X-ray detected clusters (middle; A. Finoguenov et al. 2010, in preparation), and optical groups with at least three detected members (right; Knobel et al. 2009). Each circle is centered at the position of the defined structures. Radii of the circles are scaled in R.A.-decl. plane to the number of objects in the photometric structures, X-ray luminosity for the X-ray clusters, and number of detected objects in the optical groups.

(A color version of this figure is available in the online journal.)

in the redshift interval $0.8<z<1$. Obviously, at any redshift bin and at a given $1+\delta_{p}$, the real data are affected by the cosmic variance, and we cannot see the growth in the cosmic structure over redshifts, as it is visible in the mock curves. 

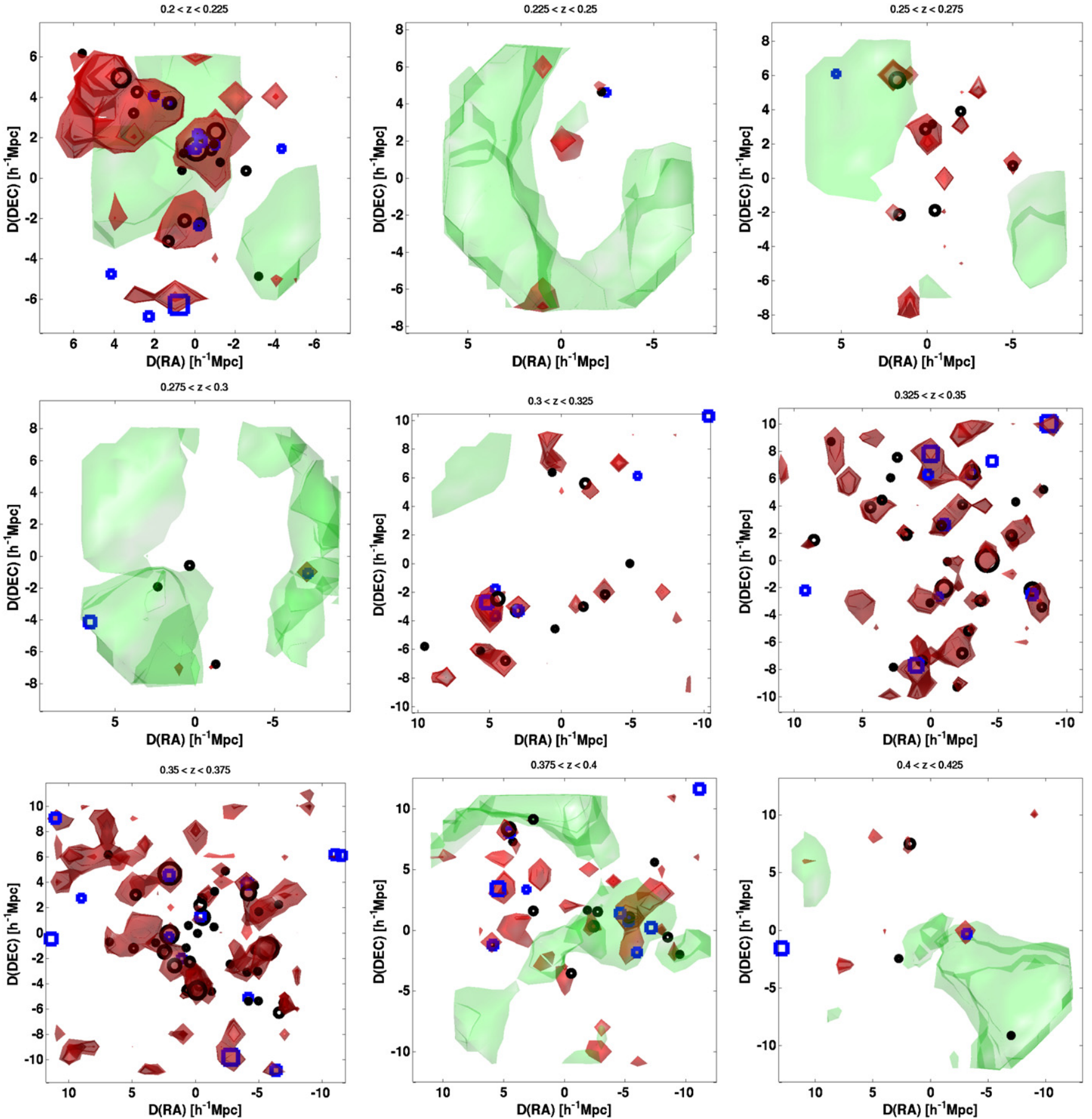

Figure 24. Overdensity field of galaxies projected in redshift slices of 0.025 width, covering the redshift interval from 0.2 to 1 . The overdensity field is reconstructed using the flux-limited sample of galaxies within the apertures defined by the fifth nearest neighbor projected within $\pm 1000 \mathrm{~km} \mathrm{~s}^{-1}$. Only structures defined by the isosurface contours of $1+\delta_{p} \geqslant 6.67$ (red or dark) and $1+\delta_{p} \leqslant 0.15$ (green or light) are shown. As a comparison, the optical groups in the same redshift bins with al least three detected members are overplotted as circles and $\mathrm{X}$-rays clusters as squares. The sizes of the symbols to mark the positions of the virialized structures are scaled as in Figure 23. The redshift slices $0.3-0.375,0.675-0.75$, and $0.875-1$ are dominated by the overdense structures. Large, R.A.-decl.-extended underdense structures are detected in the $0.45-0.5,0.525-0.55,0.575-0.6,0.625-0.675$, and $0.8-0.825$ redshift slices.

(A color version of this figure is available in the online journal.)

We follow up on this with a more detailed comparison of the distribution of "sizes" (volumes) of the structures above a given overdensity in the data and in the individual mock catalogs. We calculate the size of a structure by adding comoving volumes of the connected grid cells with overdensity above a chosen value. The grid cells need to have at least one common side (either in the R.A., decl., or $z$-direction) to be considered connected. The results are presented in Figure 27 for $1+\delta_{p} \geqslant 3$. The individual plots correspond to the survey volume fractions contained within the structures of at least the indicated size. It is noticeable that there is not a single mock catalog which contains as much volume as the real data in the largest structures in $0.2<z<1$. As a check we also recalculate the same statistics for the data when using the mean density estimated following the same 

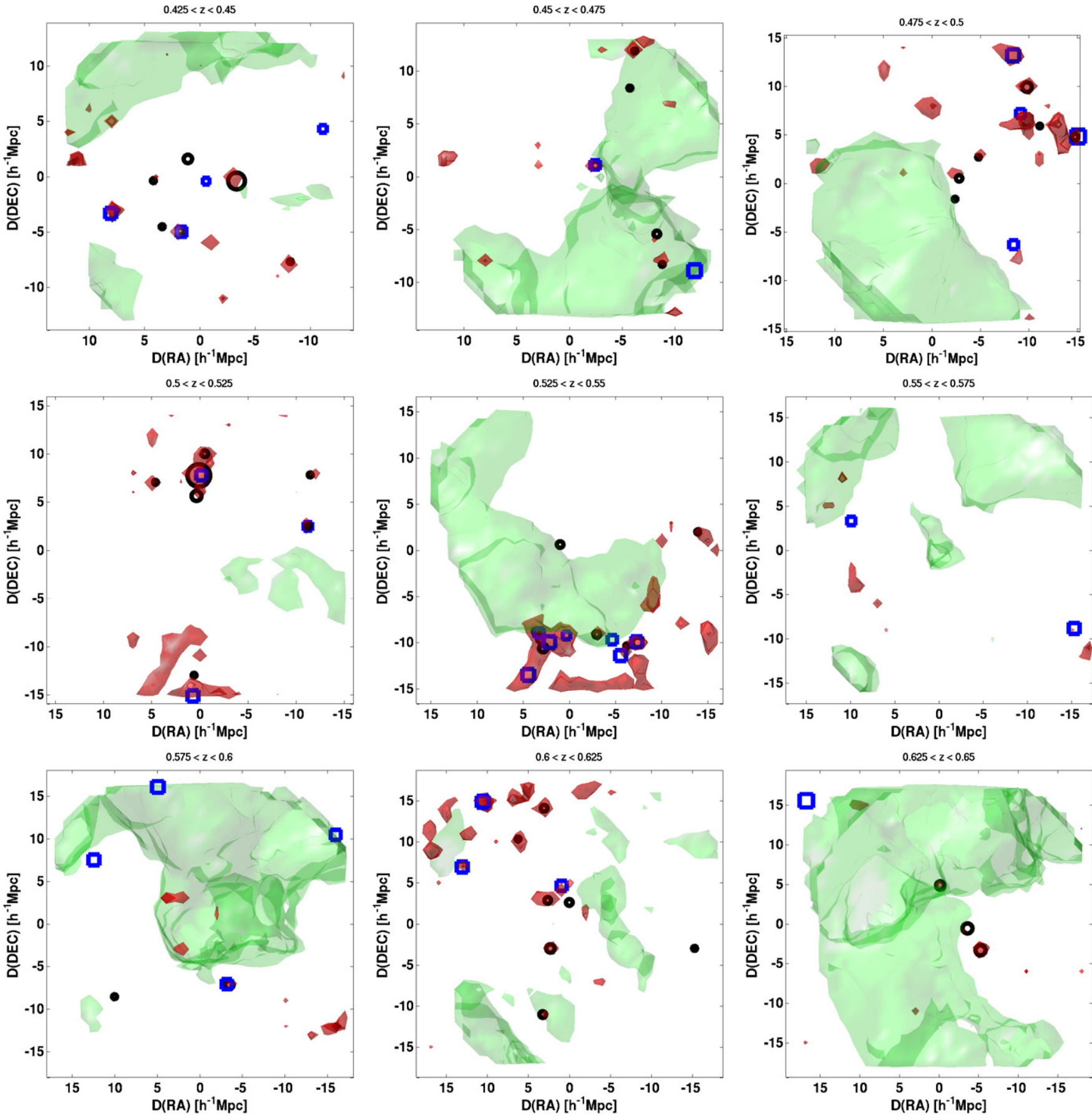

Figure 24. (Continued)

smoothing scheme as applied to the mocks (even though this smoothing is not ideal for the data). In this case, the difference between the data and the mocks is even larger.

Carrying the same analysis in the narrower redshift intervals we realize that the majority of this difference is accounted for by the large structure in $0.8<z<1$. Already, when we limit the redshift range to $0.3<z<0.9$, the data are not so different from the mocks. To conclude, except for the large structure in the highest redshift bin $0.8<z<1$, the fraction of the volume within the structures of a given size in the data and in the mock catalogs is in reasonable agreement. At the current state, given that there is a disagreement in only one $\Delta z$ slice, it is difficult to say whether the large structure in $0.8<z<1$ reflects only a cosmic variance, or it is an unusual object for the current cosmology.

\section{SUMMARY}

We have used the first $\sim 10,000$ spectra from the zCOSMOS bright survey to reconstruct the density field in the survey volume up to $z=1$. We present a new method for the reconstruction, which is based on the combination of the high-quality spectroscopic redshifts and the ZADE-modified photometric redshifts of those galaxies without spectroscopic redshifts. Our method enables us to reliably reconstruct a broader range of environments than it would be possible by using only galaxies with spectroscopic redshifts. The usefulness of this 

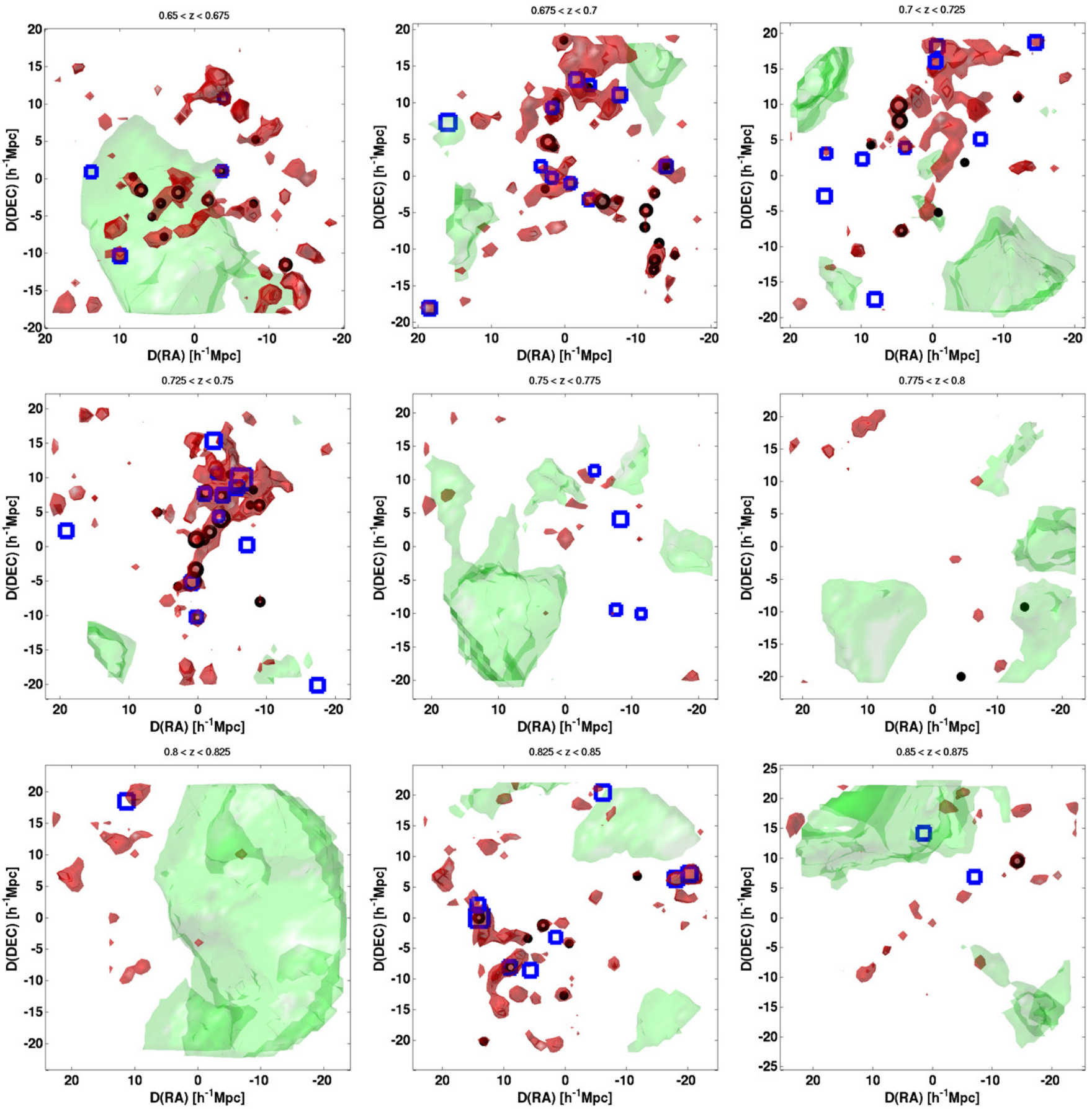

Figure 24. (Continued)

approach and its limitations are established through extensive comparisons with mock catalogs treated in exactly the same way.

We use the weighted counts of tracer galaxies within various apertures to measure the galaxy environments in the zCOSMOS volume. Given the variety of the scientific applications, we carry out the reconstruction in different ways. We construct fluxand volume-limited samples of tracer galaxies, specify fixed and adaptive apertures (characterized by distance to the $N$ th nearest neighbor), and define three-dimensional and projected distances. We weight tracer galaxies in three different ways: with unity, $B$-band luminosity, and stellar mass.

We present in detail the density field reconstructed on the grid filling the zCOSMOS volume up to $z=1$. The apertures are defined by the distance to the $N$ th nearest neighbor (fifth, 10th, and 20th) projected within $\pm 1000 \mathrm{~km} \mathrm{~s}^{-1}$ to the redshift of a point where the density is being measured. We use the fluxlimited tracer galaxies, as this sample of tracers allows us to reconstruct the density field with the broadest dynamical range and with the greatest detail of the structures. The reconstructed zCOSMOS overdensity field consists of cluster-like structures, surrounded by void-like regions, showing a complexity of the cosmic web up to $z \sim 1$.

The regions in the density field enclosed by the $1+\delta_{p} \geqslant 3$, or higher cutoff values, are well traced by the virialized objects in the zCOSMOS volume, i.e., X-ray clusters and optical groups with at least three detected members. The 

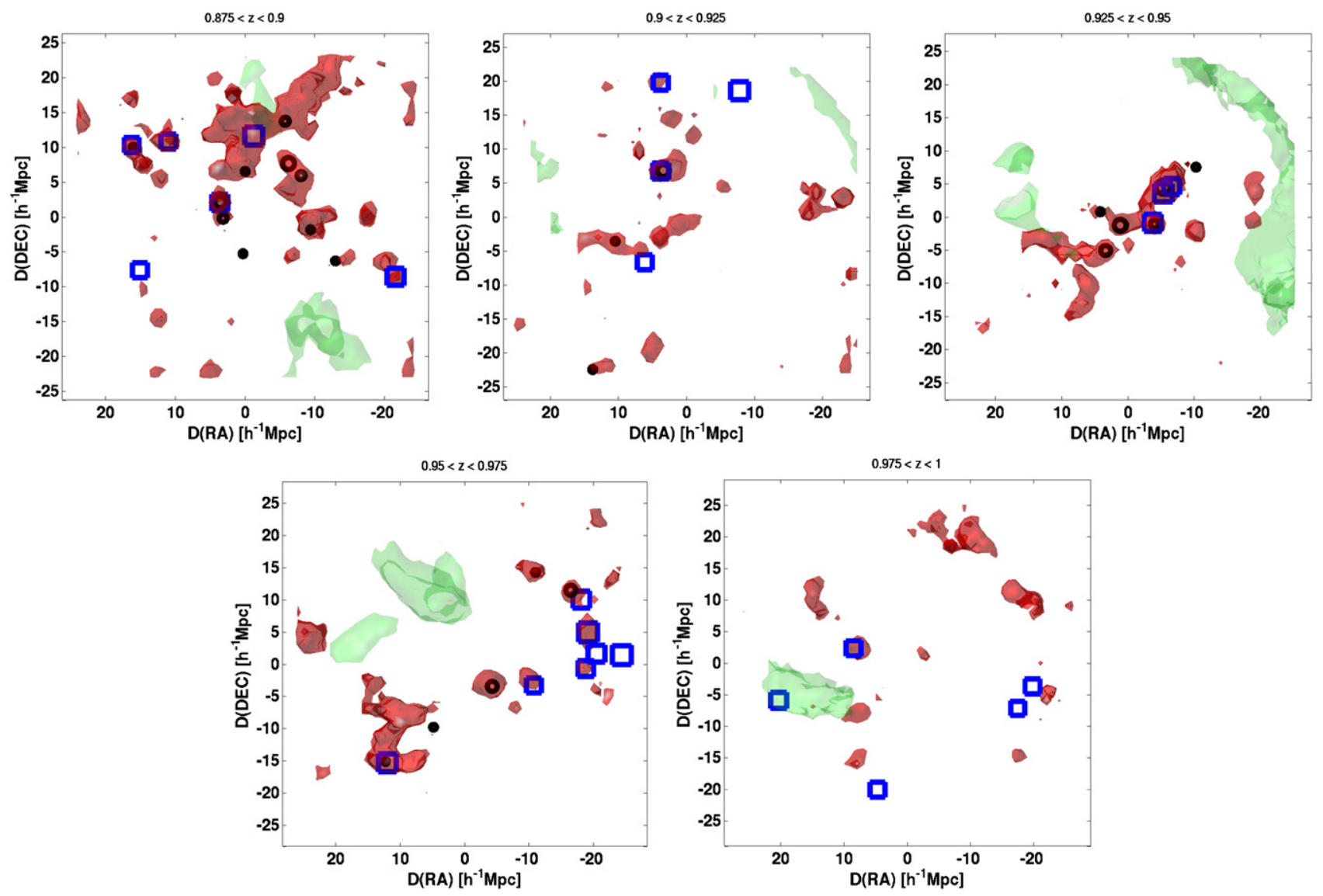

Figure 24. (Continued)
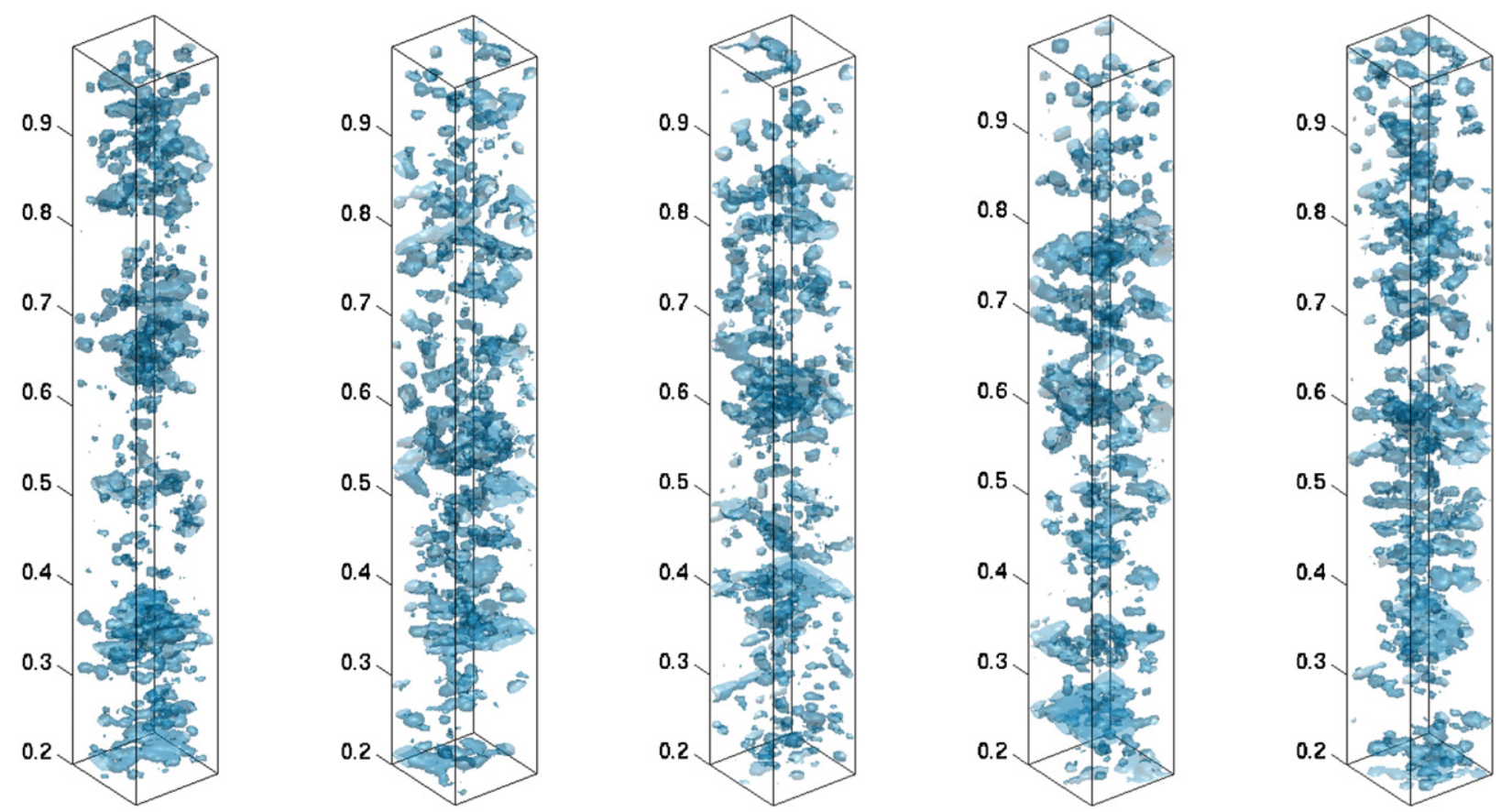

Figure 25. Comparison of the $1+\delta_{p}=3$ isosurfaces in the overdensity field reconstructed using the 10k zCOSMOS sample of flux-limited tracer galaxies and the equivalent sample of galaxies in the mock catalogs. First left-hand figure: overdensity field reconstructed using the real 10k+30kZADE zCOSMOS galaxies. Rest of figures: overdensity field reconstructed using the mock $10 \mathrm{k}+30 \mathrm{kZADE}$ samples. In every point, the overdensity field is reconstructed by counting the number of objects with an integrated ZADE-modified probability distribution in the apertures defined by the distance to the 10 th nearest neighbor projected within $\pm 1000 \mathrm{~km}$ $\mathrm{s}^{-1}$ of the redshift of the grid point. The grid is regular in $\Delta \alpha=\Delta \delta=2 \operatorname{arcmin}$ and $\Delta z=0.002$. The overdensity values are presented without the edge correction. The high complexity of the cosmic web is noticeable in all figures. It appears that there are more structures on large scales $\left(\sim 10 h^{-1} \mathrm{Mpc}\right)$, visible above $z \sim 0.8$, in the real data than in the mock catalogs. To highlight the structures in the figures, we omit the axis. The transversal axes are R.A. and decl., covering the zCOSMOS area $\sim 1 \mathrm{deg}^{2}$, and the vertical axis is redshift in the range $0.2<z<1$.

(A color version of this figure is available in the online journal.) 

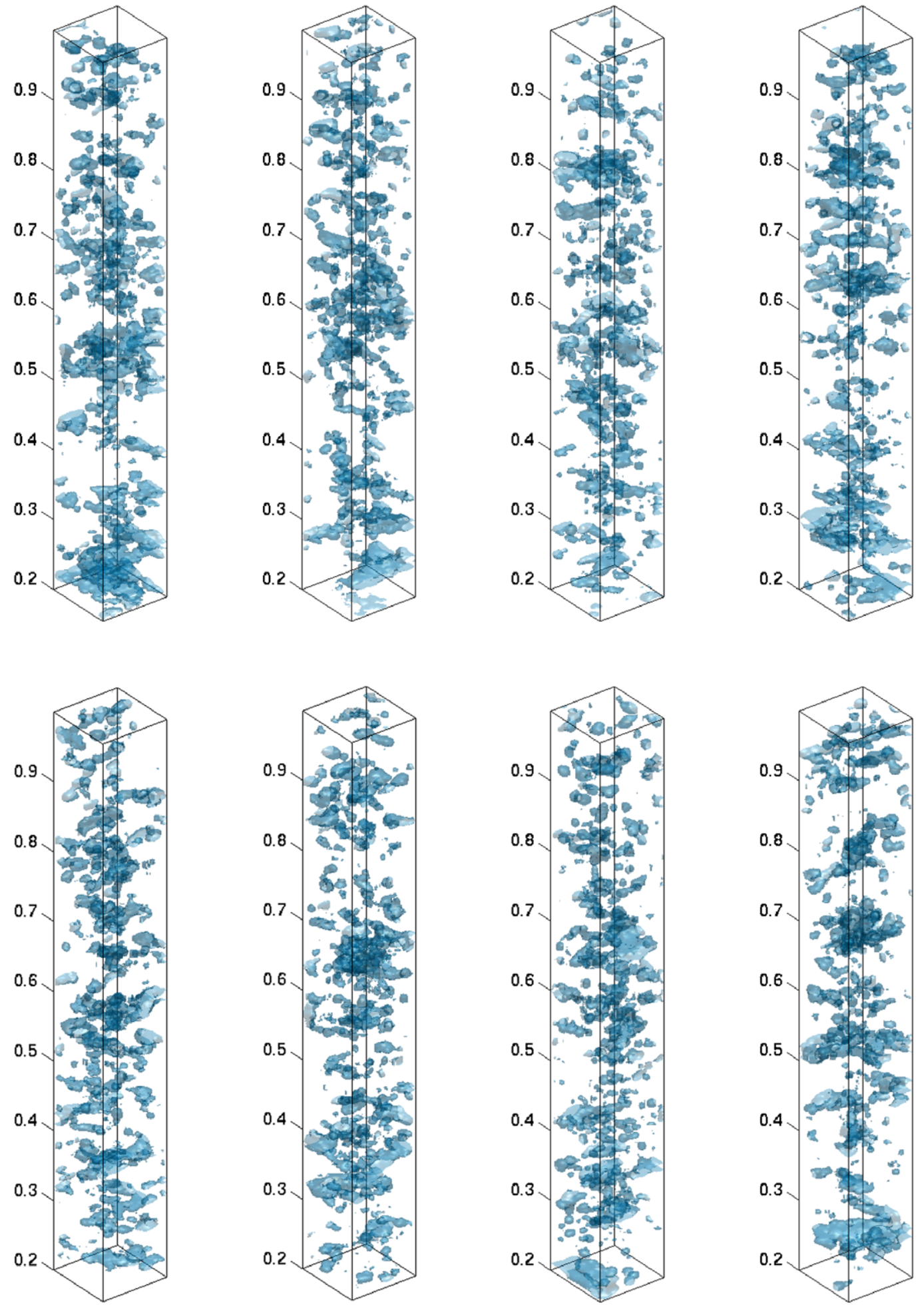

Figure 25. (Continued)

galaxies defining the poorer groups (with two or three detected members) are found to live also in much lower overdensities than the rich $N \geqslant 4$ optical groups and X-ray clusters.

Further on, we have compared the LSS in the zCOSMOS data and in the mock catalogs. There is an excellent agreement of the fractions of the volume enclosed in structures of all sizes above a given overdensity between the data and the mocks in $0.2<z<1$. However, for the chosen $1+\delta_{p}=3$ value, there is more volume enclosed in the large structures in the data than in any of the used mocks; we want to stress that this difference is mainly driven by the existence of a very large structure in the zCOSMOS, centered at $z \sim 0.9$ and extending in the radial direction over $\Delta z \sim 0.2$.

This paper is the basis for a number of studies undertaken using the zCOSMOS set of data, reported elsewhere. 

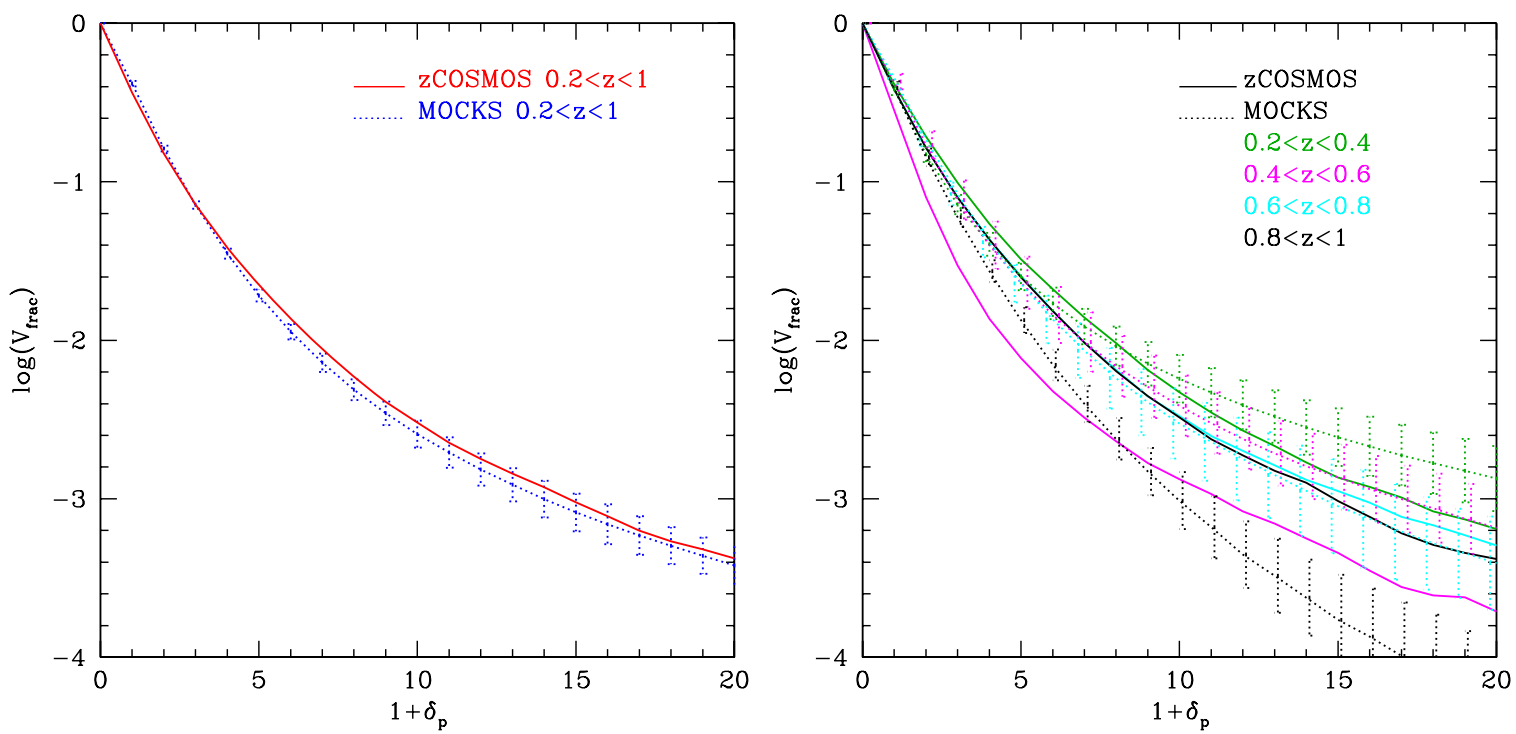

Figure 26. Fraction of the volume with the overdensity $\left(1+\delta_{p}\right)$ above a given value. The aperture in the overdensity field is defined using the distance to the 10 th nearest neighbor projected to the redshift of the grid point within $\pm 1000 \mathrm{~km} \mathrm{~s}^{-1}$. A mock value is obtained by averaging results obtained from the individual 12 mock catalogs. The error is calculated as the standard deviation from the individual mock catalog results. Volumes are estimated in $\left(h^{-1} \mathrm{Mpc}\right)^{3}$. The continuous lines represent the real data, the dotted lines represent the corresponding averaged mock catalog. Left: statistics in $0.2<z<1$. Right: statistics in four redshift bins: $0.2<z<0.4$ (green), $0.4<z<0.6$ (magenta), $0.6<z<0.8$ (cyan), and $0.8<z<1$ (black).
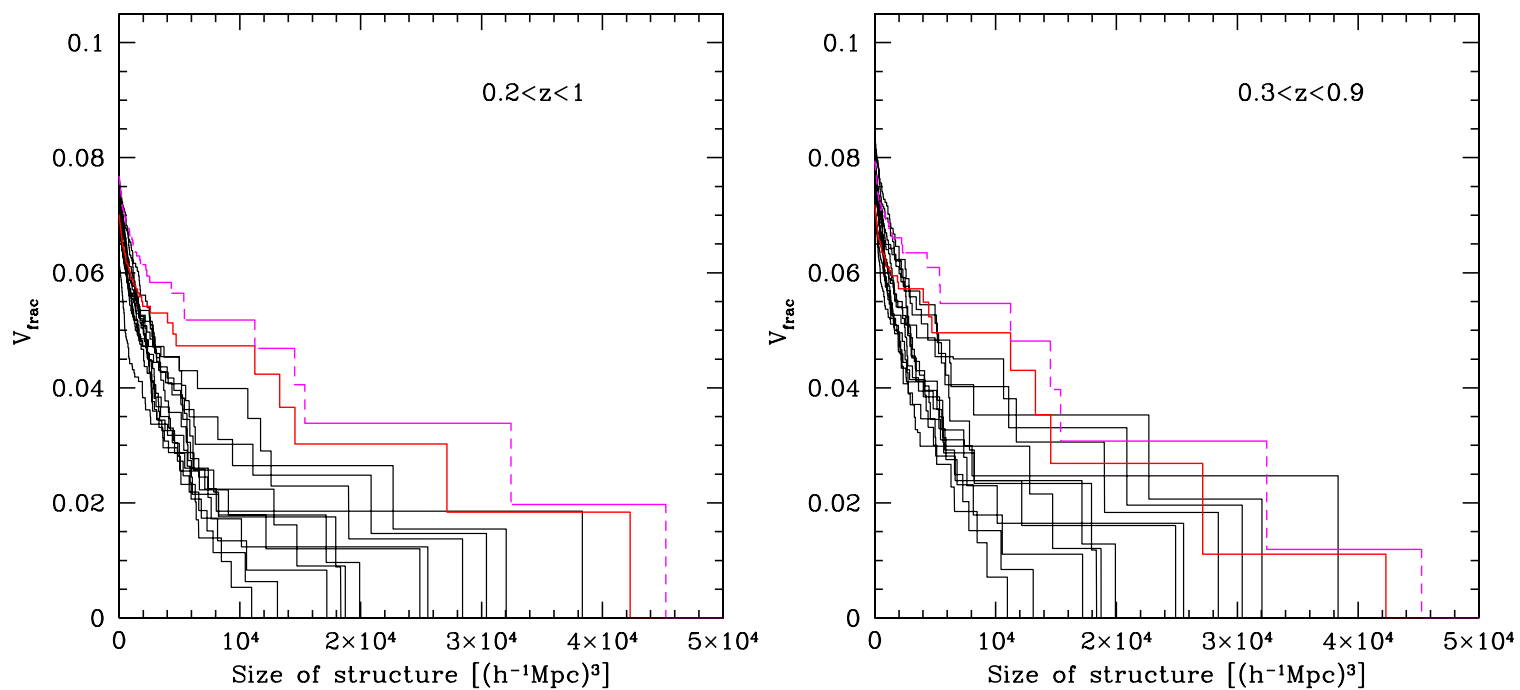

Figure 27. Comparison of the sizes of the structures $1+\delta_{p} \geqslant 3$ in the data (red: $V / V_{\max }$ smoothing to obtain $N(z)$, magenta: smoothing equivalent to the smoothing applied to the mock $N(z))$ and in the individual mock catalogs (black curves). The curves correspond to the volume fractions contained within the structures of at least the size indicated on the $x$-axis, where size is measured in $\left(h^{-1} \mathrm{Mpc}\right)^{3}$. There is not a single mock catalog which contains as much volume as the real data in the large structures in $0.2<z<1$. When we limit our statistics to $0.3<z<0.9$, the data results fall within the statistics outlined by the mocks. Difference between the data and the mocks at the large sizes is dominated by the structure at $z \sim 0.9$.

This work has been supported in part by a grant from the Swiss National Science Foundation and by grant ASI/COFIS/WP3110I/026/07/0. We thank M. G. Kitzbichler and S. D. M. White for providing the mock catalogs (Kitzbichler \& White 2007).

\section{REFERENCES}

Aragón-Calvo, M. A., Jones, B. J. T., van de Weygaert, R., \& van der Hulst, J. M. 2007, A\&A, 474, 315

Bernardeau, F., \& van de Weygaert, R. 1996, MNRAS, 279, 693

Blanton, M. R., Eisenstein, D., Hogg, D. W., \& Zehavi, I. 2006, ApJ, 645, 977

Blanton, M. R., et al. 2003, ApJ, 594, 186

Bolzonella, M., et al. 2009, A\&A, submitted, arXiv:0907.0013

Bond, J. R., Kofman, L., \& Pogosyan, D. 1996, Nature, 380, 603

Bruzual, G., \& Charlot, S. 2003, MNRAS, 344, 1000
Calzetti, D., Armus, L., Bohlin, R. C., Kinney, A. L., Koornneef, J., \& StorchiBergmann, T. 2000, ApJ, 533, 682

Capak, P., et al. 2007, ApJS, 172, 99

Caputi, K. I., et al. 2009, ApJ, 691, 91

Chabrier, G. 2003, PASP, 115, 763

Coil, A. L., Newman, J. A., Cooper, M. C., Davis, M., Faber, S. M., Koo, D. C., \& Willmer, C. N. A. 2006, ApJ, 644, 671

Coil, A. L., et al. 2008, ApJ, 672, 153

Coles, P., \& Jones, B. 1991, MNRAS, 248, 1

Colless, M., et al. 2001, MNRAS, 328, 1039

Cooper, M. C., Newman, J. A., Madgwick, D. S., Gerke, B. F., Yan, R., \& Davis, M. 2005, ApJ, 634, 833

Cooper, M. C., et al. 2007, MNRAS, 376, 1445

Croton, D. J., et al. 2006, MNRAS, 365, 11

Cucciati, O., et al. 2006, A\&A, 458, 39

Cucciati, O., et al. 2009, A\&A, submitted

Davis, M., et al. 2003, Proc. SPIE, 4834, 161

de Lapparent, V., Geller, M. J., \& Huchra, J. P. 1986, ApJ, 302, L1

de la Torre, S., et al. 2007, A\&A, 475, 443 
De Lucia, G., \& Blaizot, J. 2007, MNRAS, 375, 2

Delone, B. N. 1934, Bull. Acad. Sci. USSR, 7, 793

Dirichlet, G. L. 1850, J. Reine Angew. Math., 40, 209

Dressler, A. 1980, ApJ, 236, 351

Feldmann, R., et al. 2006, MNRAS, 372, 565

Finoguenov, A., et al. 2007, ApJS, 172, 182

Forero-Romero, J. E., Hoffman, Y., Gottloeber, S., Klypin, A., \& Yepes, G. 2009, MNRAS, 396, 1815

Guzzo, L., et al. 2007, ApJS, 172, 254

Hahn, O., Carollo, C. M., Porciani, C., \& Dekel, A. 2007, MNRAS, 381, 41

Hahn, O., Porciani, C., Carollo, C. M., \& Dekel, A. 2007, MNRAS, 375, 489

Hernquist, L., \& Katz, N. 1989, ApJS, 70, 419

Hoffman, Y. 1994, in ASP Conf. Ser. 67, Unveiling Large-scale Structures Behind the Milky Way, ed. C. Balkowski \& R. C. Kraan-Korteweg (San Francisco, CA: ASP), 185

Ilbert, O., et al. 2009, ApJ, 690, 1236

Kauffmann, G., White, S. D. M., Heckman, T. M., Ménard, B., Brinchmann, J., Charlot, S., Tremonti, C., \& Brinkmann, J. 2004, MNRAS, 353, 713

Kitzbichler, M. G., \& White, S. D. M. 2007, MNRAS, 376, 2

Kovač, K., Somerville, R. S., Rhoads, J. E., Malhotra, S., \& Wang, J. 2007, ApJ, 668,15

Kovač, K., et al. 2009, ApJ, submitted (arXiv:0910.0004)

Knobel, C., et al. 2009, ApJ, 697, 1842

Lahav, O., Fisher, K. B., Hoffman, Y., Scharf, C. A., \& Zaroubi, S. 1994, ApJ, 423, L93

Le Fèvre, O., et al. 2005, A\&A, 439, 845

Lee, K.-S., Giavalisco, M., Gnedin, O. Y., Somerville, R. S., Ferguson, H. C., Dickinson, M., \& Ouchi, M. 2006, ApJ, 642, 63

Lee, J., \& Lee, B. 2008, ApJ, 688, 78

Lee, J., \& Li, C. 2008, MNRAS, submitted (arXiv:0803.1759)

Lilly, S. J., et al. 2007, ApJS, 172, 70

Lilly, S. J., et al. 2009, ApJS, 184, 218

Marinoni, C., et al. 2005, A\&A, 442, 801

Marinoni, C., et al. 2008, A\&A, 487, 7

Massey, R., et al. 2007, Nature, 445, 286
Meneux, B., et al. 2008, A\&A, 478, 299

Meneux, B., et al. 2009, A\&A, 505, 463

Norberg, P., et al. 2001, MNRAS, 328, 64

Norberg, P., et al. 2002, MNRAS, 332, 827

Novikov, D., Colombi, S., \& Doré, O. 2006, MNRAS, 366, 1201

Ouchi, M., et al. 2003, ApJ, 582, 60

Owen, J. M., Villumsen, J. V., Shapiro, P. R., \& Martel, H. 1998, ApJS, 116 155

Park, C., Choi, Y.-Y., Vogeley, M. S., Gott, J. R. I., \& Blanton, M. R. 2007, ApJ, 658, 898

Pozzetti, L., et al. 2009, A\&A, submitted (arXiv:0907.5416)

Romano-Díaz, E., \& van de Weygaert, R. 2007, MNRAS, 382, 2

Salvato, M., et al. 2009, ApJ, 690, 1250

Schaap, W. E. 2007, PhD thesis, Rijksuniversiteit, Groningen

Schaap, W. E., \& van de Weygaert, R. 2000, A\&A, 363, L29

Scoville, N., et al. 2007a, ApJS, 172, 38

Scoville, N., et al. 2007b, ApJS, 172, 150

Shapiro, P. R., Martel, H., Villumsen, J. V., \& Owen, J. M. 1996, ApJS, 103, 269

Sigad, Y., Branchini, E., \& Dekel, A. 2000, ApJ, 540, 62

Silverman, J. D., et al. 2009, ApJ, 695, 171

Sousbie, T., Pichon, C., Colombi, S., Novikov, D., \& Pogosyan, D. 2008, MNRAS, 383, 1655

Springel, V., et al. 2005, Nature, 435, 629

Strauss, M. A., \& Willick, J. A. 1995, Phys. Rep., 261, 271

Taniguchi, Y., et al. 2007, ApJS, 172, 9

Tasca, L. A. M., et al. 2009, A\&A, 503, 379

van Breukelen, C., et al. 2006, MNRAS, 373, L26

van de Weygaert, R., \& Bertschinger, E. 1996, MNRAS, 281, 84

Vergani, D., et al. 2009, A\&A, submitted (arXiv:0909.1968)

Voronoi, G. 1908, J. Reine Angew. Math., 134, 198

York, D. G., et al. 2000, AJ, 120, 1579

Zaroubi, S., Hoffman, Y., Fisher, K. B., \& Lahav, O. 1995, ApJ, 449, 446

Zehavi, I., et al. 2005, ApJ, 630, 1

Zucca, E., et al. 2009, A\&A, in press (arXiv:0909.4674) 